WHOI-85-19

Copy 1

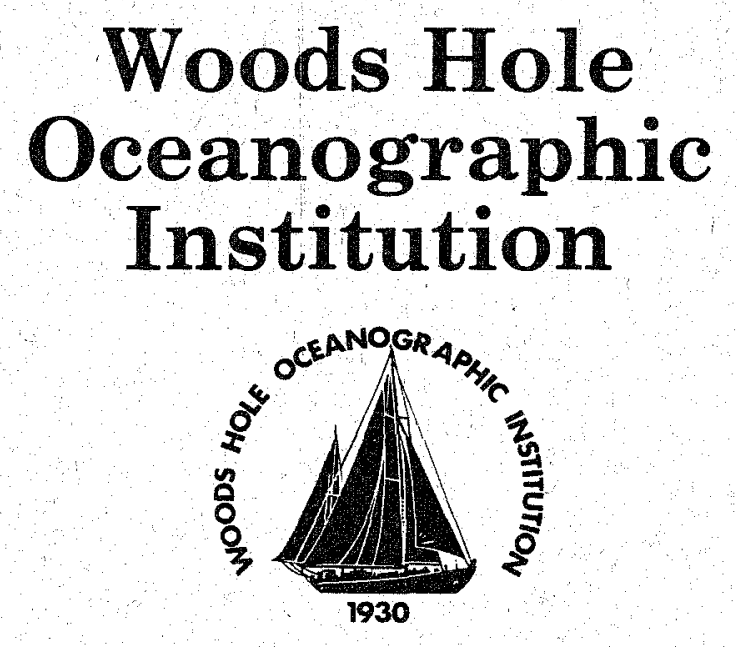

\title{
Fallout Nuclides in Atlantic and Pacific Water Columns: GEOSECS Data
}

by

H. D. Livingston, V. T. Bowen, S. A. Casso, H. L. Volchok, V. E. Noshkin, K. M. Wong, and T. M. Beasley

May 1985

Technical Report

Funding was provided by the United States Department of Energy under Contract DE-ACO2-EV03563.

Approved for public release; distribution unlimited. 


\title{
Fallout Nuclides in Atlantic and Pacific Water Columns: GEOSECS Data
}

\author{
by \\ H. D. Livingston 1 , V. T. Bowen ${ }^{1}$, S. A. Casso ${ }^{1}$, H. L. Volchok ${ }^{2}$, \\ V. E. Noshkin ${ }^{3}$, K. M. Wong ${ }^{3}$, and T. M. Beasley 4 \\ at \\ 1. Woods Hole Oceanographic Institution \\ Woods Hole, MA 02543 \\ 2. Environmental Measurements Laboratory, U.S. Dept. of Energy, \\ 376 Hudson Street, New York, NY 10014 \\ 3. Lawrence Livermore Laboratory, University of California, \\ P.O. Box 808 (L-233), Livermore, CA 94550 \\ 4. School of Oceanography, Oregon State University, \\ Marine Science Center, Newport, OR 97365 \\ May 1985 \\ Technical Report \\ Funding was provided by the U.S. Department of Energy under \\ contract DE-AC02-EV03563. \\ Reproduction in whole or in part is permitted for any purpose of the \\ United States Government. This report should be cited as: \\ Woods Hole Oceanog. Inst. Tech. Rept., WHOI-85-19. \\ Approved for publication; distribution unlimited.
}

\section{Approved for Distribution:}

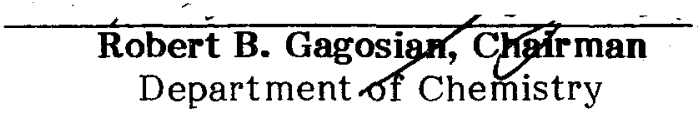




$\begin{array}{lr}\text { Abstract } & i \text { i } \\ \text { Introduction } & 1 \\ \text { Sampling, Methods and Quality Control } & 5 \\ \text { Results } & 8 \\ \text { Acknowledgements } & 9 \\ \text { References } & 11 \\ \text { SECrION 1 - Radiochemical Data (GEOSECS Atlantic) } & 13 \\ \text { Cruise Track and Station Locations (GEOSECS Atlantic) } & 47 \\ \text { SECTION 2 - Radiochemical Data (GEOSECS Pacific) } & 48 \\ \text { Cruise Track and Station Locations (GEOSECS Pacific) }\end{array}$




\section{Abstract}

This report contains results of measurements of the fallout radionuclides ${ }^{90} \mathrm{Sr},{ }^{137} \mathrm{Cs},{ }^{239,240} \mathrm{Pu}$, and ${ }^{241} \mathrm{Am}$ in large volume seawater samples collected between 1972 and 1974 in the Atlantic and Pacific as part of Geochemical Ocean Sections (GEOSECS) program. The stations for which data are reported include both the North and South Atlantic oceans and latitudes north of $20^{\circ} \mathrm{S}$ in the Pacific Ocean. The ${ }^{90} \mathrm{Sr}$ and ${ }^{137} \mathrm{Cs}$ data set has been corrected by a procedure which estimates independently the analytical blank for the laboratory which made the analysis. When the data quality and spacing permit, water column inventory estimates were made for each nuclide over depth intervals appropriate to the nuclide's distribution. 


\section{Introduction}

The GEOSECS (Geochemical Ocean Sections) program, which began to be funded by the National Science Foundation in 1971, was the first really large-scale, multi-ocean, multi-laboratory and multi-discipline attempt to combine high quality physical oceanographic measurements with chemical measurements of both natural and anthropogenic chemical substances, both stable and radioactive, in the wor1d's oceans. Broadly stated, it had a goal of trying to understand the physical and biogeochemical controls which determine the properties and behavior of chemical substances in the ocean. Through 1979, the program produced five volumes of collected papers published in Earth and Planetary Science Letters (e.g. Craig and Turekian, 1980) and hydrographic data tables and atlases published by the National Science Foundation (Bainbridge et al., 1981-83). A further series of data reports and atlases devoted to the shore-based chemical measurements is planned for the near future.

This report has been prepared to tabulate the results obtained by the various laboratories of the concentrations and distributions in the Atlantic and Pacific 0cean of some of the bomb-derived fallout radionuclides introduced to the oceans subsequent to atmospheric nuclear weapons tests. This report contains data obtained on samples collected as part of the GEOSECS program in these oceans and is part of the continuing study of these substances in the world ocean and of their use as tracers to study a variety of physical and biogeochemical oceanic processes.

The radionuclides for which data are reported include the fission product radionuclides ${ }^{137} \mathrm{Cs}$ and ${ }^{90} \mathrm{Sr}$ and the transuranic elements $\mathrm{Pu}$ and $\mathrm{Am}$ - produced by neutron capture and decay chain processes. This suite of radioele- 
ments encompasses a range of chemical reactivities with respect to particle scavenging - ranging from the soluble end $\left({ }^{137} \mathrm{Cs}\right.$ and $\left.{ }^{90} \mathrm{Sr}\right)$, where the processes controlling the nuclide distributions are primarily physical, to the reactive end $\left({ }^{241} \mathrm{Am}\right)$, where uptake and transport on settling particles becomes an important process. Plutonium appears to lie somewhere in the middle of the range. Initially it was believed to exhibit relatively high particle reactivity but recent studies are beginning to point to a lower degree of particle reactivity than was originally postulated. As chemical analogues, ${ }^{137} \mathrm{Cs}$ and ${ }^{90} \mathrm{Sr}$ would be hard to distinguish from ${ }^{3} \mathrm{H}$, based on their observed oceanic distribution patterns, while ${ }^{241}$ Am appears likely to have a chemical reactivity approaching that of $\mathrm{Th}$.

A discussion of distribution patterns of fallout radionuclide patterns in the Pacific, based primarily on GEOSECS data, provided a first large-scale review, in a single ocean, of the fate of these fallout radionuclides (Bowen et a1., 1980). It was stated in this paper that the individual data upon which the paper was based would be published in a forthcoming report. Partly, then, this report is intended to complete this plan. In addition, it includes the available data set for fallout nuclides measured in Atlantic GEOSECS samples and a suite of ${ }^{241} \mathrm{Am}$ data which were not discussed in the above paper.

One comment may be appropriate at this point in respect to the quality of the reported data, although this is discussed 1ater. As would be expected, data quality deteriorates as ambient concentrations approach sensitivity limits. As the input of fallout has varied to the oceanic areas for which data are reported here, the uncertainties in the data, especially in the deeper samples, increase as the level of the fallout input decreases. The fallout 
input decreased in the order North Pacific $>$ North Atlantic $>$ South Atlantic. An additional factor which resulted in the quality of the transuranic data in the Pacific samples being higher than that of the Atlantic samples is the fact that the Pacific samples were generally analyzed at a later time than were the Atlantic ones. Improved chemical recoveries, increased detector availability, and hence counting times, all worked to produce a Pacific transuranic data set of significantly higher precision than that obtained for the Atlantic samples.

\section{Sampling, Methods and Quality Control}

A fairly detailed account of the technigues used in collection and fallout radionuclide analyses of the large volume water samples from the Atlantic and Pacific GEOSECS cruises appeared in a paper discussing the Pacific data set (Bowen et a1., 1980). This account, though directed at the samples collected on the Pacific GEOSECS cruises, is also generally relevant to the collection and analyses of Atlantic GEOSECS samples. We therefore include here an abbreviated outline of these procedures, supplemented by remarks which are relevant to the fuller data set presented in this report.

In both Atlantic and Pacific GEOSECS cruises, large volume sample collection for fallout nuclide analysis was made primarily by the 260 liter Gerard samplers. After ${ }^{14} \mathrm{C}$ extraction, 60 liter samples were returned to shorebased laboratories for fallout nuclide analyses - in some cases, following Rn extraction for ${ }^{226} \mathrm{Ra}$ analysis. Fallout radionuclide analyses were subsequently completed in a variety of combinations of radionuclides analyzed by a given laboratory. Four different laboratories performed or contracted the various radiochemical analyses. 
(1) Some samples were analyzed by commercial laboratories, under contract to the (now) U. S. Dept. of Energy, Environmental Measurements Laboratory in New York City, NY. H. L. Volchok, of that laboratory, supervised the contracting and reporting. These analyses were restricted to measurement of ${ }^{90} \mathrm{Sr}$ alone or for ${ }^{137} \mathrm{Cs}$ and ${ }^{90} \mathrm{Sr}$.

(2) Some samples were analyzed by V. E. Noshkin's group at Lawrence Livermore Laboratories (LLL). Mostly ${ }^{137} \mathrm{Cs}$ and $\mathrm{Pu}$ isotopes were determined by LLL although a few sample series were processed for ${ }^{137} \mathrm{Cs}$ and ${ }^{90} \mathrm{Sr}$ by a contractor laboratory after LLL isolated a Pu fraction.

(3) Some samples were analyzed by V. T. Bowen and H. D. Livingston's group at Woods Hole Oceanographic Institution (WHOI). These analyses typically included ${ }^{137} \mathrm{Cs},{ }^{90} \mathrm{Sr}, \mathrm{Pu}$ isotopes and, for a subset, ${ }^{241} \mathrm{Am}$.

(4) A number of samples, from the Atlantic GEOSECS sample suite, were analyzed for Pu and ${ }^{241}$ Am by T. M. Beasley's laboratory at Oregon State University (OSU). A small number of these samples were, in addition, processed to isolate a $\mathrm{Cs}$ fraction at OSU, and then the radiochemistry and sample counting completed at WHOI.

The analytical methods used by these various laboratories have been described in detail elsewhere (if not proprietary) and are referenced in Bowen et a1. (1980). The methods used by the OSU laboratory were generally similar to those used by WHOI or LLL with the exception of a solvent extraction based radiochemical procedure for ${ }^{241} \mathrm{Am}$.

Throughout the analytical program for these GEOSECS samples, a considerable analytical effort was devoted to activities which provided assurance of data quality. These efforts are again described in detail by Bowen et al. 
(1980) and include the analyses of known 'blind' samples, duplicates, environmental blanks, reagent blanks and interlaboratory intercomparison exercise samples. In addition, both Atlantic and Pacific data sets have been subjected to a "deep ocean blank" procedure for blank correcting measured values for ${ }^{137} \mathrm{Cs}$ and ${ }^{90} \mathrm{Sr}$ obtained both by different laboratories and at different times. Details of this procedure have been reported for Atlantic samples by Kupferman and Livingston (1979) and essentially the same approach was followed for blank correction to the GEOSECS Pacific data set.

$\underline{\text { Resu1ts }}$

Radiochemical and hydrographic data from GEOSECS Atlantic and Pacific stations are compiled in the Tables of this report. The first set of tables lists the data for most of the Atlantic GEOSECS large volume stations. The second set 1ists data from Pacific GEOSECS large volume stations north of $20^{\circ} \mathrm{S}$. In some cases no samples for fallout nuclide analyses were collected at South Pacific stations, or were collected by methods which did not protect the integrity of the sample with respect to contamination during storage; some South Pacific large volume samples remain unanalyzed for reasons of lack of funding or scientific interest.

Each large volume station is identified by its GEOSECS station number preceded by the prefix GX. Hydrographic data for the large volume water samples were derived from the GEOSECS Atlantic and Pacific preliminary leg reports. Depths are best depths in meters. Salinities and potential temperatures are in conventional units; densities are 1 isted as sigma theta. Concentrations of ${ }^{137} \mathrm{Cs},{ }^{90} \mathrm{Sr},{ }^{239}, 240 \mathrm{Pu}$ and ${ }^{241} \mathrm{Am}$ are tabulated where measured in units of disintegrations per $100 \mathrm{~kg}$ of seawater as of date of collection. One sigma 
counting uncertainties are 1 isted in the columns labelled by the letter e (error). $239,240 \mathrm{Pu}$ concentrations are tabulated under the abbreviated Pu239 heading but are conventionally the sum of ${ }^{239} \mathrm{Pu}$ and ${ }^{240} \mathrm{Pu}$ concentrations unresolvable by state-of-the-art alpha spectrometry. In a few cases no uncertainty is listed with a value for a radionuclide. For a variety of reasons no estimate of the counting uncertainty was obtained and the value listed should be regarded as an upper limit (or a less than value) for the actual concentration in the sample.

The final column in the tables lists the laboratory code which has been used to identify the laboratory or laboratories which performed the radiochemical analyses of each large volume sample. As described in the previous section, there were a variety of combinations of radiochemical analyses used over the several years during which the lengthy analyses were completed. A listing of these various combinations and of the laboratories involved is tabulated in Table 1 .

Also listed in the tables are the results of integration of the various radionuclide concentrations measured over all or part of the water column at each station. The result of this calculation is an estimate of the radionuclide inventory in the water column at each station over the depth intervals over which these calculations were made. The inventory units are expressed as $\mathrm{mCi} / \mathrm{km}^{2}$. Where possible, the integration was carried out over the complete water column, but in many cases this was not possible or appropriate. At some stations or for some radionuclides the available data were not complete enough to define the water column distribution well enough to permit a meaningful inventory calculation. The other limiting factor was frequently analytical sen- 
sitivity. In the deep ocean, especially in areas where atmospheric fallout input was minimal, the concentrations at depths below the main thermocline were often essentially zero or at the limit of available analytical sensitivities. In this situation, small overestimates of nuclide concentrations in the generally large portion of the water column below the main thermocline would result in substantial and erroneous overestimates of the actual nuclide inventory. The inventory calculations 1 isted are therefore data limited and the depth intervals over which integration was made is noted at each station and for each nuclide. Deep water inventory calculations were also susceptible to overestimation when concentrations were close to sensitivity limits because of the nature of the inventory integration calculation itself. Firstly, no consideration was given to the precision of a given concentration - only the actual concentration value being used. At low deep water concentrations, the uncertainties in the measurement often produced apparent concentrations substantially higher than was reasonable, but with very large uncertainties. When these situations occurred, inventory overestimates were likely to be non-trivial. Secondly, only positive concentration values were computed in the inventory calculation. Blank correction procedures often produced negative concentration values for some nuclides from deep water. Exclusion of these again tends to bias inventory estimates. The integration calculation itself used the measured concentration of a nuclide at a given depth to represent the average concentration over the depth interval between the mid-points of the distances to the sample depths above and below the given depth.

The radionuclides ${ }^{90} \mathrm{Sr}$ and ${ }^{137} \mathrm{Cs}$ have been found to exhibit oceanic chemistries dominated by highly soluble characteristics such that their dis- 
tributions are essentially controlled by purely physical processes of mixing (Kupferman and Livingston, 1979). Their oceanic distributions thus are genera11y found to correlate strongly with those of other soluble tracers introduced from the atmosphere with comparable temporal and spatial input histories - such as tritium, or to lesser extent, freons. Except in oceanic areas which have received recent deep ventilation, the distribution of these species is for practical purposes confined to depths shallower than the bottom of the main thermocline. Accordingly, integration of ${ }^{137} \mathrm{Cs}$ and ${ }^{90} \mathrm{Sr}$ distributions at such stations was in general only made to a depth where their concentrations became undetectable. The distributions of the particle active transuranic elements $239,240 \mathrm{Pu}$ and ${ }^{241} \mathrm{Am}$ were integrated where the data permitted throughout the whole water column.

\section{Acknowledgements}

The collection and publication of the data in this report was made possible through the efforts of a large group of people who are too numerous to list here. They include the officers, crew and science parties on research vessels Knorr and Melville and the many radiochemists in the several laboratories represented who generated the basic data set. S. L. Kupferman and D. E. Moore provided the capability of the 'deep ocean blank' correction technique applied to ${ }^{90} \mathrm{Sr}$ and ${ }^{137} \mathrm{Cs}$ data. Without all of this skilled help, this work would not have been possible and it is our pleasure to acknowledge it here.

Support for this work has been variously funded as follows: at WHOI, subcontracts from EYL, and directly to WHOI from the U. S. Department of Energy under contract $73-\mathrm{C}-02-3563$; at LLL, under the auspices of the U. S. Depart- 
ment of Energy under contract number W-7405-ENG-48. We are very grateful to these agencies and to the several program administrators for this support.

\section{References}

Bainbridge, A. E. (1981-3) GEOSECS Atlantic and Pacific Expeditions, Hydrographic data, Sections and Profiles. Vol. 1-4. National Science Foundation, Washington, DC.

Bowen, V. T., V. E. Noshkin, H. D. Livingston and H. L. Volchok (1980) Fallout radionuclides in the Pacific Ocean: vertical and horizontal distributions, largely from GEOSECS stations. Earth Planet. Sci. Lett. $\underline{49}$, 411-434.

Craig, H. and K. K. Turekian (1980) The GEOSECS program: 1976-1979. Earth P1anet. Sci. Lett. 49 , 263-265.

Kupfarman, S. L. and H. D. Livingston (1979) A procedure for independent1y estimating blanks and uncertainties for measured values of ${ }^{90} \mathrm{Sr}$ and ${ }^{137}$ Cs concentrations in the Atlantic Ocean. J. Mar. Res. 37, 141-156. 
TABLE 1

Radionuclide Analysis by Laboratory

\begin{tabular}{|c|c|c|c|c|}
\hline Lab Code & $\mathrm{Cs}$ & $\mathrm{Sr}$ & $\underline{\mathrm{Pu}}$ & Am \\
\hline $\mathbf{J}$ & $W$ & $\mathrm{~T}$ & $\mathbf{W}$ & $\mathbf{W}$ \\
\hline $\mathbf{K}$ & $\mathbf{T}$ & $T$ & $W$ & $\mathbf{W}$ \\
\hline L & L & $\mathrm{L}$ & L & \\
\hline 0 & 0 & 0 & 0 & 0 \\
\hline $\mathbf{P}$ & $W$ & $T$ & 0 & 0 \\
\hline$Q$ & $\mathbf{T}$ & $\mathbf{T}$ & L & \\
\hline $\mathbf{R}$ & 0 & $T$ & 0 & 0 \\
\hline$S$ & $\mathrm{~T}$ & $\mathbf{T}$ & 0 & 0 \\
\hline $\mathrm{T}$ & $\mathrm{T}$ & $\mathbf{T}$ & & \\
\hline$t$ & $\mathrm{t}$ & $t$ & & \\
\hline $\mathbf{U}$ & 0 & $t$ & 0 & \\
\hline V & $\mathbf{W}$ & $\mathrm{T}, \mathrm{W}^{\star}$ & $\mathbf{W}$ & $\mathbf{W}$ \\
\hline $\mathbf{W}$ & $\mathbf{W}$ & $W$ & $\mathbf{W}$ & $\mathbf{W}$ \\
\hline$x$ & $W$ & $W, W^{*}$ & $\mathbf{W}$ & $\mathbf{W}$ \\
\hline $\mathbf{Y}$ & $w, t^{n}$ & $w, t^{n}$ & W & $\mathbf{W}$ \\
\hline Z & L & $\mathrm{T}$ & L & \\
\hline
\end{tabular}

$\mathrm{L}$ = Lawrence Livermore Laboratory

$0=$ Oregon State University

$T$ = Trape10 West (now Environmental Analysis Laboratory)

$t=$ Teledyne

$W=$ Woods Hole Oceanographic Institution

* = Value reported is the mean of two measurements 
$-11-$

SECTION 1

Radiochemical Data (GEOSECS Atlantic) 
$-12-$

\section{-}




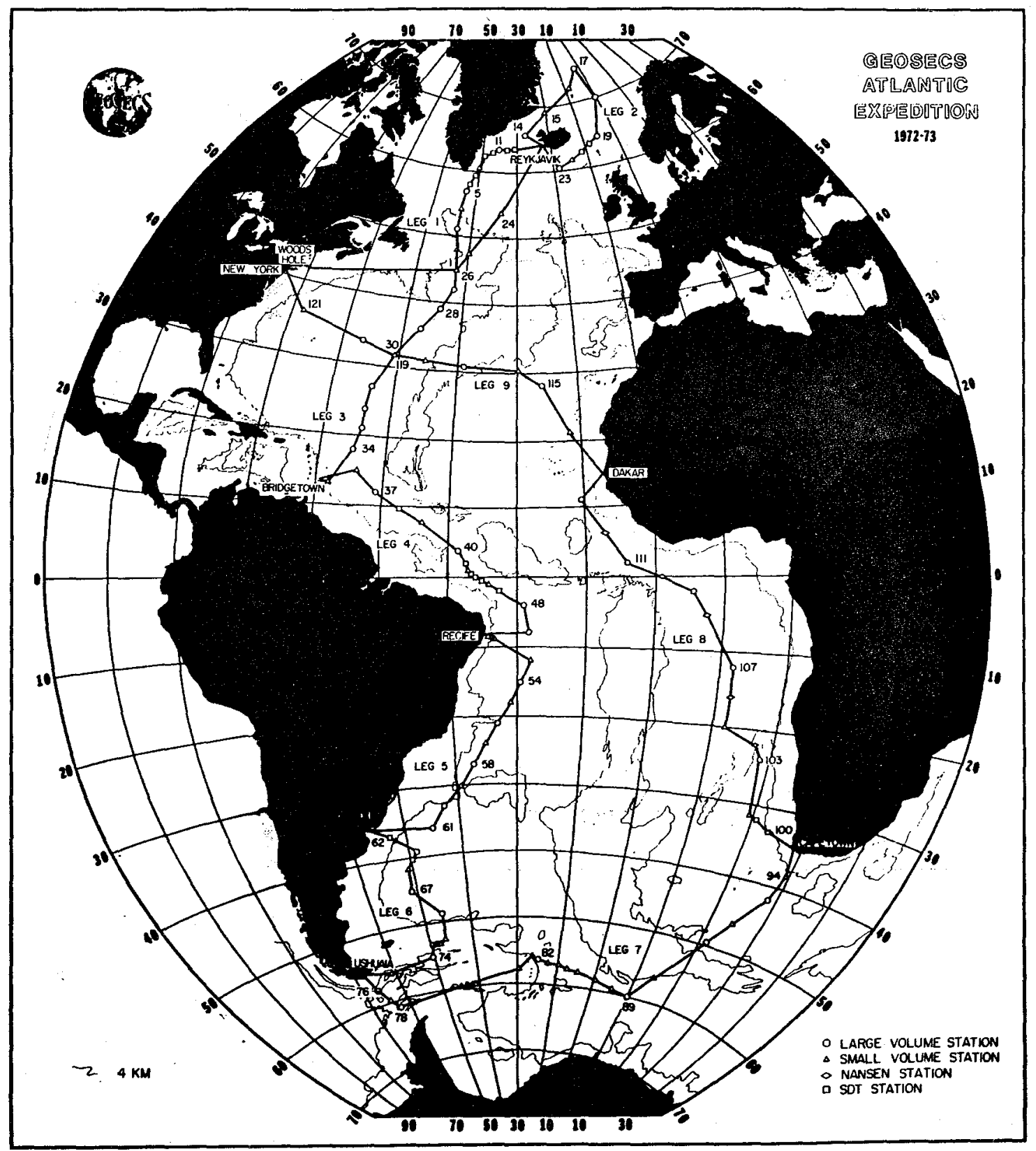




\begin{tabular}{|c|c|c|c|c|c|c|c|c|c|c|c|}
\hline \multicolumn{2}{|c|}{ CFUISE-STA\# } & \multicolumn{2}{|c|}{ P0SIIIOH } & \multicolumn{2}{|c|}{ COLLECTION DATE } & \multicolumn{2}{|c|}{ GOTTON DEPTH } & & & & \\
\hline \multirow[t]{2}{*}{$6:-3$} & & 1.0 & H & \multicolumn{2}{|c|}{$07 / 28 / 72$} & \multirow{2}{*}{\multicolumn{2}{|c|}{$4321 \mathrm{M}$}} & & & & \\
\hline & & 1.0 & H & & & & & & & & \\
\hline \multirow[t]{2}{*}{ DEFTH } & \multirow[t]{2}{*}{ SALIHITY } & \multirow[t]{2}{*}{ POT. T } & SIGHA & \multicolumn{2}{|c|}{65137} & \multicolumn{2}{|c|}{ SR90 } & \multicolumn{2}{|c|}{ FII 239} & AM241 & LAE \\
\hline & & & THETA & & e & & E & & $\mathrm{e}$ & $\Xi$ & cote \\
\hline \multicolumn{12}{|c|}{ 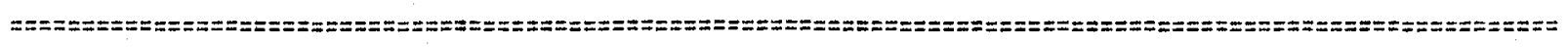 } \\
\hline 0 & 34.347 & & & 30.6 & 0.6 & 22.2 & 0.3 & 0.120 & 0.030 & & W \\
\hline 71 & 35.062 & & & 13.8 & 1.0 & $1 b .2$ & 0.9 & & & & $T$ \\
\hline 225 & 34.884 & 7.380 & 27.315 & 14.3 & 0.6 & 10.4 & 0.5 & ด. 169 & 0.030 & & n \\
\hline 303 & 34.923 & 6.996 & 27.401 & 18.0 & 1.1 & 13.0 & 1.1 & & & & $T$ \\
\hline 511 & 34.866 & 5.230 & 27.582 & 12.5 & 0.6 & 10.6 & 0.2 & 0.140 & 0.030 & & 4 \\
\hline 661 & 34.965 & & & 13.6 & 0.9 & 7.9 . & 1.2 & & & & I \\
\hline 814 & 34.960 & & & 16.2 & 0.6 & 11.1 & 0.4 & 0.220 & 0.040 & & $H$ \\
\hline 1117 & 34.948 & 4.030 & 27.779 & 16.0 & 1.0 & 11.6 & 1.4 & & & & 1 \\
\hline 1321 & 34.945 & & & 13.7 & 0.8 & 8.7 & 0.2 & 0.200 & 0. 040 & & $\#$ \\
\hline 2948 & 34.953 & 3.480 & 27.846 & 5.8 & 0.9 & 2.9 & 0.6 & 0.060 & 0.020 & & $W$ \\
\hline 2786 & 34.946 & & & 4.7 & 0.9 & 2.3 & 0.9 & & & & $T$ \\
\hline 3279 & 34.936 & 2.330 & 27.927 & 4.9 & 1.0 & 3.8 & 0.1 & 0.170 & 0.050 & & $\#$ \\
\hline 3792 & 34,919 & & & 8.3 & 0.6 & 4.4 & 0.2 & 9.100 & 0.020 & & 4 \\
\hline 4306 & 34.920 & 1.630 & 27.968 & 12.1 & 1.1 & 8.9 & 0.4 & 0.190 & 0.070 & & 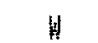 \\
\hline
\end{tabular}

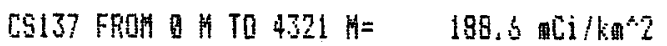

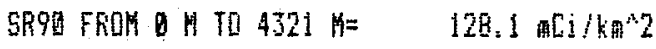

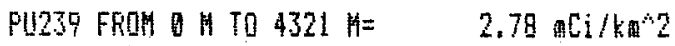




\begin{tabular}{|c|c|c|c|c|c|c|c|c|c|c|}
\hline \multicolumn{2}{|c|}{ ERUISE-STAH } & \multicolumn{2}{|c|}{ PDSITION } & \multicolumn{2}{|c|}{ COLLECTION DATE } & \multicolumn{2}{|c|}{ BOTTOM DEPTH } & & & \\
\hline \multirow[t]{2}{*}{$6 x-5$} & & it 54.0 & H & \multicolumn{2}{|c|}{$07 / 31 / 72$} & \multirow{2}{*}{\multicolumn{2}{|c|}{$3390 \mathrm{M}$}} & & & \\
\hline & & $12 \quad 47.0$ & H. & & & & & & & \\
\hline \multirow[t]{2}{*}{ DEPTH } & \multirow[t]{2}{*}{ SALIHITY } & \multirow[t]{2}{*}{ P0T. T } & SIGMA & \multicolumn{2}{|c|}{ C\$137 } & \multicolumn{2}{|c|}{ SR90 } & FU23? & AM241 & LAE \\
\hline & & & THETA & & $\varepsilon$ & & $\mathrm{e}$ & e & $\mathrm{e}$ & COUE \\
\hline \multicolumn{11}{|c|}{ 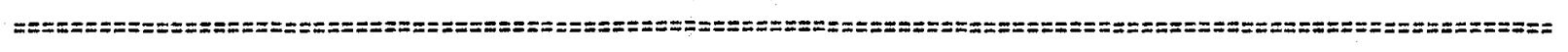 } \\
\hline 5 & 34.130 & & & 18.4 & 1.0 & 15.7 & 0.9 & & & $\mathrm{t}$ \\
\hline 100 & 34.745 & & & & & 12.6 & 0.5 & & & $t$ \\
\hline 200 & 34.837 & & & 25.8 & 1.2 & 12.4 & 0.3 & & & $t$ \\
\hline 307 & 34.879 & 4.010 & 27.727 & 24.4 & 1,3 & 11.8 & 0.7 & & & $t$ \\
\hline 350 & 34.849 & & & & & 13.7 & 9.6 & & & $\mathrm{t}$ \\
\hline 615 & 34.860 & & & & & 14,8 & 0.7 & & & $t$ \\
\hline 922 & 34.866 & 3.430 & 27.773 & 11.2 & 0.7 & 9.2 & 0.4 & & & $t$ \\
\hline 1230 & 34.933 & & & & & 7.6 & 0.4 & & & $\mathrm{t}$ \\
\hline 1643 & 34.940 & & & 3.6 & 0.4 & 2.0 & 0.3 & & & $\mathrm{t}$ \\
\hline 2052 & 34.955 & 3.200 & 27.866 & & & 3.2 & 0.2 & & & $t$ \\
\hline 2672 & 34.962 & 2.750 & 27.912 & 9.1 & 0.5 & 3.3 & O. 4 & & & $\mathrm{t}$ \\
\hline 2877 & 34.943 & & & & & 3.7 & 0.4 & & & $t$ \\
\hline 3982 & 34.915 & & & 6.2 & 0.4 & 4.6 & 0.4 & & & $t$ \\
\hline 3287 & 34.924 & & & & & 6.0 & 0.3 & & & $t$ \\
\hline
\end{tabular}

CS137 FROM O $M$ TO $3390 M=$ SR9Q FFIH O T TO $3390 \mathrm{H}=$
141.2 aCi $/ \mathrm{km}^{\wedge} 2$

$103.1 \mathrm{aCi} / \mathrm{k}^{2} 2$ 


\begin{tabular}{|c|c|c|c|c|c|c|c|c|c|c|c|}
\hline \multicolumn{2}{|c|}{ CFUISE-STA } & \multicolumn{2}{|c|}{ Position } & \multicolumn{2}{|c|}{ COLLECTION DATE } & \multicolumn{2}{|c|}{ BOTTOH DEPTH } & & & & \\
\hline \multirow[t]{2}{*}{$6 x-11$} & & $63 \quad 30.0$ & N & \multicolumn{2}{|c|}{$98 / 95 / 72$} & \multirow{2}{*}{\multicolumn{2}{|c|}{2392 แ }} & & & & \\
\hline & & $35 \quad 14.0$ & 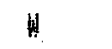 & & & & & & & & \\
\hline \multirow[t]{2}{*}{ DEPTH } & \multirow[t]{2}{*}{ SALIHITY } & \multirow[t]{2}{*}{ POT. T } & SIGHA & \multicolumn{2}{|c|}{$\mathrm{\$} 137$} & \multicolumn{2}{|c|}{$5 R 90$} & \multicolumn{2}{|c|}{ FUn? 39} & AM241 & $\angle A B$ \\
\hline & & & THETÁ & & e & & e & & e & e & CODE \\
\hline \multicolumn{12}{|c|}{ 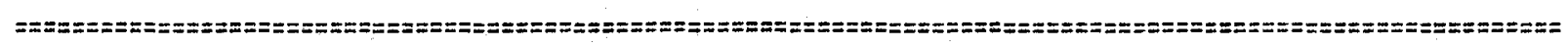 } \\
\hline $\mathrm{a}$ & 34.956 & & & 20.5 & 0.6 & 13.9 & 0.4 & 0.210 & 0.030 & & $H$ \\
\hline $2 \theta$ & 34.966 & & & 21.1 & 1.0 & 10.2 & 1.1 & 0.080 & 0.010 & & a \\
\hline 100 & 35.635 & & & 18.9 & 0.6 & 12.7 & 0.7 & 0.120 & 0.040 & & 4 \\
\hline 203 & 35.034 & 5.830 & 27.641 & 18.7 & 1.1 & 10.5 & 1.2 & 0.169 & 0.010 & & 0 \\
\hline 365 & 34.957 & & & 19.3 & 0.5 & 12.3 & 9.8 & 0.300 & 0.050 & & $H$ \\
\hline 400 & 34.935 & 4.600 & 27.708 & 17.4 & 0.9 & 14.2 & 0.9 & & & & $\mathrm{~T}$ \\
\hline 612 & 34.922 & & & 18.3 & 0.6 & 12.4 & 0.5 & 0.090 & 0.030 & & H \\
\hline 819 & 34.915 & & & 13.6 & 3.0 & 11.2 & 6.9 & 0.220 & 0.050 & & 0 \\
\hline 1026 & 34.911 & & & 14.7 & 0.8 & 10.6 & 0.3 & 0.230 & 0.050 & & H \\
\hline 1232 & 34.923 & 3.690 & 27.793 & 12.8 & 0.9 & 8.5 & 0.8 & 0.160 & 0.010 & & Q \\
\hline 1440 & 34.953 & & & 7.9 & 0.6 & 5.6 & 0.3 & 0.110 & 0.030 & & 4 \\
\hline 1646 & 34.948 & 3.490 & 27.834 & 7.8 & 0.9 & 4.1 & 1.2 & & & & $T$ \\
\hline 1822 & 34.966 & & & 3.9 & 0.7 & 4.1 & 0.4 & $0.09 \%$ & 0.030 & & W \\
\hline 2027 & 34.936 & & & 8.6 & 1.1 & 7.4 & 0.9 & 0.130 & 0.010 & & 0 \\
\hline 2130 & 34.926 & & & 9.9 & 1.8 & 7.9 & 0.3 & 0.110 & 0.020 & & 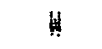 \\
\hline 2233 & 34.915 & & & 13.7 & 1.1 & 10.5 & 1.7 & & & & $\ddot{T}$ \\
\hline
\end{tabular}

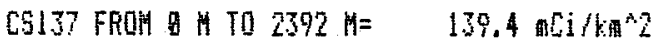
5990 Froh O H TO 2392 H= PU237 FAOM OA TO $2392 \mathrm{M}=$
$99.3 \mathrm{mCi} / \mathrm{km} \mathrm{m}^{\wedge} 2$

$1.61 \mathrm{mCi} / \mathrm{km}^{2} 2$ 


\begin{tabular}{|c|c|c|c|c|c|}
\hline CRUISE-STAH & \multicolumn{3}{|c|}{ POSITION } & COLLECTION DATE & BOTTOH DEPTH \\
\hline $6 x-14$ & 65 & 55.7 & $H$ & $08 / 13 / 72$ & $646 M$ \\
\hline & 27 & 27.0 & 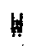 & & \\
\hline
\end{tabular}

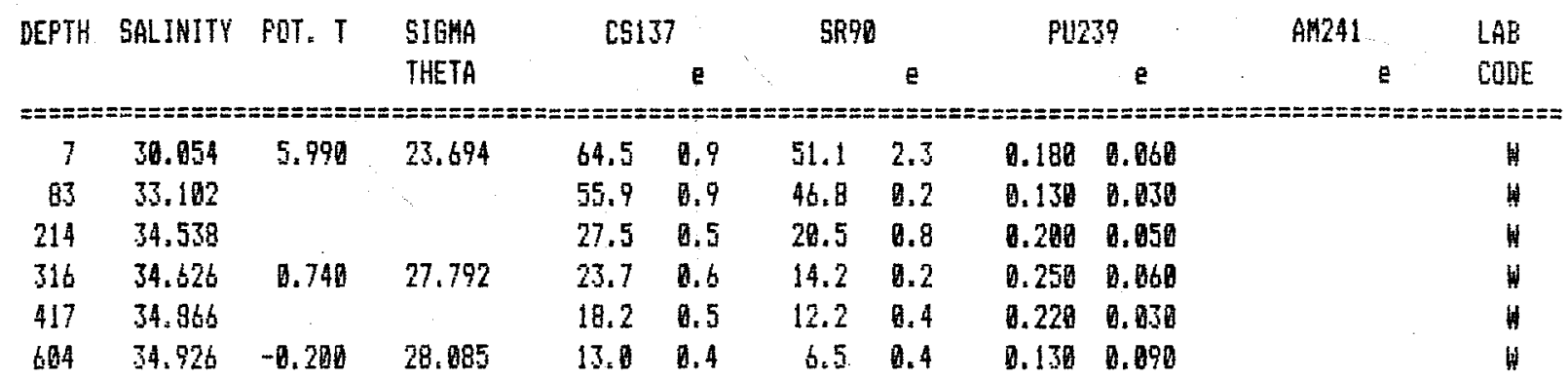

C5137 FROM D TO $646 \mathrm{H}=$

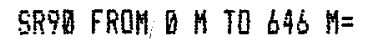
PU239 FFOM I I TO $646 \mathrm{H}=$
84.1 $\mathrm{aCi} / \mathrm{kn} 2$

$61.3 \mathrm{aCl} / \mathrm{km}^{2} 2$

$0.54 \mathrm{aCi} / \mathrm{k}$. 2 


\begin{tabular}{|c|c|c|c|c|c|c|c|c|c|c|c|c|}
\hline \multicolumn{2}{|c|}{ CRUISE-STA } & \multicolumn{2}{|c|}{ POSITION } & \multicolumn{2}{|c|}{ COLLECTION DATE } & \multicolumn{2}{|c|}{ BOTTOM DEPTH } & & & & & \\
\hline \multirow[t]{2}{*}{$6 \%-17$} & $\cdots$ & $4 \quad 56.2$ & N & \multirow{2}{*}{\multicolumn{2}{|c|}{$08 / 18 / 72$}} & \multirow{2}{*}{\multicolumn{2}{|c|}{$3740 \mathrm{M}$}} & & & & & \\
\hline & & 7.2 & W & & & & & & & & & \\
\hline \multicolumn{13}{|c|}{$3560 M=3470+3582+35234$, COMPOSITE } \\
\hline \multirow[t]{2}{*}{ DEPTH } & \multirow[t]{2}{*}{ SALINITY } & \multirow[t]{2}{*}{ POT. T } & SIGMA & \multicolumn{2}{|c|}{$[5137$} & \multirow[t]{2}{*}{ SR9 } & & \multicolumn{2}{|c|}{ PU239 } & \multicolumn{2}{|c|}{ AM241 } & LAB \\
\hline & & & THETA & & e & & e & & e & & e & CODE \\
\hline \multicolumn{13}{|c|}{ 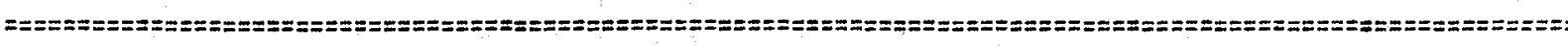 } \\
\hline 4 & 34.479 & 4.700 & 27.335 & 20.5 & 0.3 & 12.1 & 0.5 & 0.130 & 0.050 & 0.380 & 0.200 & $x$ \\
\hline 49 & 34.750 & -0.930 & 27.974 & 16.1 & 0.4 & 11.6 & 0.4 & 0.850 & 0.020 & & & $y$ \\
\hline 76 & 34.819 & -1.720 & 28.049 & 19.1 & B. 4 & 10.9 & 0.2 & 0.180 & 0.040 & & & $\|$ \\
\hline 153 & 34.831 & -1.660 & 28,064 & 18.5 & 0.5 & 10.9 & 0.3 & 0.170 & 0.920 & 8.020 & 0. 010 & $y$ \\
\hline 230 & 34.855 & $-1,320$ & 28.073 & 16.3 & D.5 & 9.8 & 0.1 & 0.140 & 0.930 & & & H \\
\hline $30 b$ & 34.873 & -1.110 & 28.080 & 12.8 & 0.4 & 9.2 & 0.2 & 0.190 & 0.030 & 0.030 & 0.020 & $y$ \\
\hline 511 & 34.966 & & & 10.4 & 0.4 & 7.0 & 0.2 & B.199 & 0.040 & & & " \\
\hline 820 & 34.900 & -1.210 & 28.106 & 4.5 & 0.2 & 4.1 & 0.2 & 0.890 & 0.020 & 0.010 & 0.810 & $Y$ \\
\hline 1025 & 34.901 & -1.140 & 28.104 & 6.6 & 0.4 & 3.3 & 0.2 & 0.048 & 0.020 & & & W \\
\hline 1332 & 34.901 & -1.200 & 28.106 & 3.7 & 0.6 & 2.8 & 0.1 & & & & & $t$ \\
\hline 1833 & & & & 6.6 & 0.5 & 3.5 & 0.2 & 9.080 & 0.0 .30 & & & $\|$ \\
\hline 2079 & 34.896 & & & 3.7 & 0.3 & 4.1 & 0.2 & 0.090 & 0.010 & & & $y$ \\
\hline 2386 & 34.891 & & & 6.2 & 0.4 & 3.7 & 0.4 & 0.890 & 0.030 & & & 4 \\
\hline 2592 & 34.890 & -1.320 & 28.101 & 4.3 & 0.3 & 4.0 & 0.2 & 0.060 & 0.010 & & & Y \\
\hline 3955 & 34.896 & -1.310 & 28.098 & 5.7 & 0.6 & 3.5 & 0.1 & 9.040 & 0.030 & & & 4 \\
\hline 3069 & 34.887 & & & 3.4 & 0.5 & 6.4 & 0.5 & & & & & $\mathrm{t}$ \\
\hline 3261 & 34,893 & -1.320 & 29.096 & 5.8 & 0.8 & 4.5 & 0.2 & 0.020 & 0.020 & & & 4 \\
\hline 3416 & 34.891 & -1.320 & 28.102 & 5.8 & 0.4 & 3.3 & 0.2 & 0.050 & 0.010 & & & Y \\
\hline 3560 & & & & 7,1 & 0.3 & 3.7 & 0.6 & 0.138 & 0.021 & & & 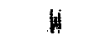 \\
\hline
\end{tabular}

[5137 FROM O TO $3740 \mathrm{H}=113.6 \mathrm{aCj} / \mathrm{km} 2$

SPOO FROM OH TO $3740 \mathrm{H}=\quad 79.5 \mathrm{mC \textrm {m }} / \mathrm{km}^{\times 2}$

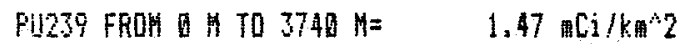




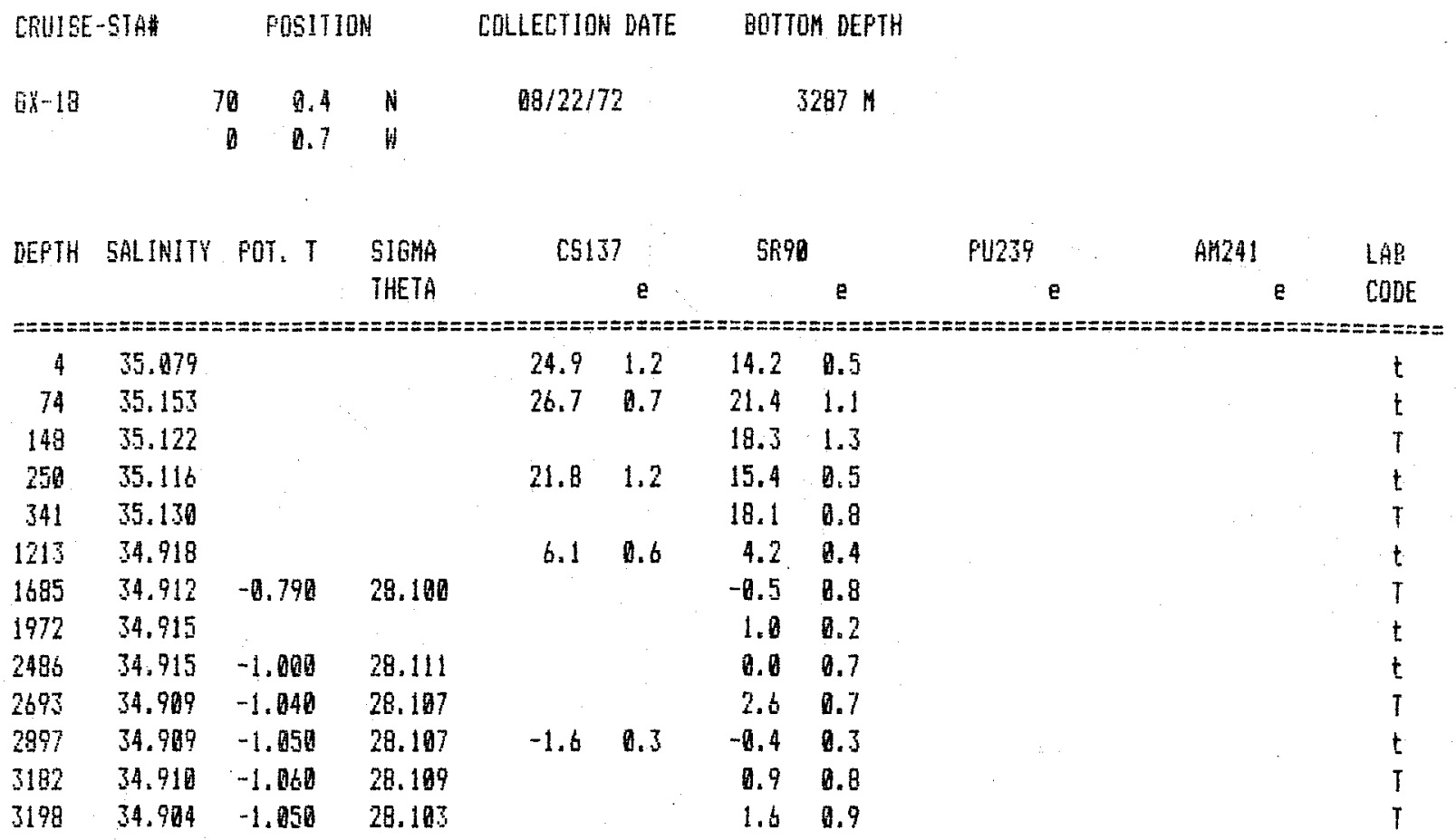

CS137 FFOM O IO $1500 \mathrm{M}=$ 5F9日 FROH o To $1500 \mathrm{M}=$
96.2 mCi $/ \mathrm{kn} 2$

$75.4 \mathrm{~m}[\mathrm{i} / \mathrm{km} / 2$ 


\begin{tabular}{|c|c|c|c|c|c|c|c|c|c|c|c|}
\hline \multicolumn{2}{|c|}{ CRUISE-STA\# } & \multicolumn{2}{|c|}{ POSITION } & \multicolumn{2}{|c|}{ COLLECTION DATE } & \multicolumn{2}{|c|}{ BOTTOM DEPTH } & & & & \\
\hline $6 y-19$ & & $54 \quad 10.0$ & $N$ & $08 / 25 /$ & & & $3399 \mathrm{H}$ & & & & \\
\hline \multirow[t]{2}{*}{ DEPTH } & \multirow[t]{2}{*}{ SALIHITY } & \multirow[t]{2}{*}{ POT. T } & SIGMA & \multicolumn{2}{|c|}{$[5137$} & \multicolumn{2}{|c|}{ SR99 } & \multicolumn{2}{|c|}{ Fu239 } & AM241 & LAB \\
\hline & & & THETA & & e & & e & & e & e & CODE \\
\hline \multicolumn{12}{|c|}{ 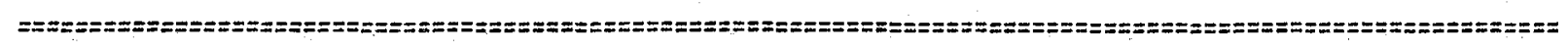 } \\
\hline 4 & 35.045 & 10.310 & 26.973 & 21.9 & 0.6 & 14.7 & 0.5 & 0.100 & 0.120 & & W \\
\hline 66 & 35.146 & 6.820 & 27.599 & 25.5 & 0.9 & 13.8 & 0.3 & 0.190 & 0.030 & & $H$ \\
\hline 144 & 34.990 & 4.750 & 27.735 & 23.6 & 1.0 & 19.2 & 1.2 & & & & $T$ \\
\hline 247 & 34.918 & 3.630 & 27.795 & 26.3 & 0.9 & 17.6 & 0.4 & 0.330 & 0.040 & & $H$ \\
\hline 349 & 34.971 & 2,020 & 27.949 & 27.3 & 1.4 & 16.3 & 2.1 & & & & $T$ \\
\hline 458 & 35.014 & 1.870 & 28.026 & 27.6 & 1.0 & 17.4 & 0.3 & 0.230 & 0.830 & & $H$ \\
\hline 559 & 34.997 & 1.440 & 28.044 & 26.2 & 1.1 & 17.9 & 0.8 & & & & $\mathrm{T}$ \\
\hline 663 & 34.963 & 0.840 & 28.057 & 22.4 & 0.4 & 13.6 & 1.3 & 0.220 & 0.850 & & W \\
\hline 765 & 34,930 & & & 17.4 & 0.9 & 11.8 & 1.1 & & & & $T$ \\
\hline 866 & 34.919 & -0.100 & 28.074 & 12.7 & 0.5 & 7.4 & 0.2 & 0.110 & 0.030 & & $H$ \\
\hline 969 & 34.913 & -0.360 & 28.082 & & & 4.2 & 0.9 & & & & $T$ \\
\hline 1113 & 34.918 & -0.550 & 28.095 & 1.6 & 0.6 & 0.4 & 0.3 & 0.010 & 0.010 & & 4 \\
\hline 1532 & 34,917 & -0.860 & 28.107 & 1.3 & 0.9 & 0.7 & 1.2 & & & & $T$ \\
\hline 2455 & 34.918 & $-1,050$ & 28.115 & 0.4 & 1.0 & 1.8 & 1.6 & & & & $T$ \\
\hline 2866 & 34.914 & -1.850 & 28.112 & 0.6 & 0.4 & 1.0 & 0.6 & 0.030 & & & 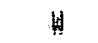 \\
\hline 378 & 34.915 & -1.860 & 28.113 & 0.0 & 1.0 & 1.9 & 0.9 & & & & $T$ \\
\hline
\end{tabular}

C5137 FFOM O $10390 \mathrm{M}=$ SR90 FROM ON TO 3390 H=

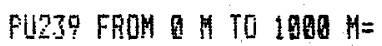

$198.1 \mathrm{mCi} / \mathrm{km} 2$

$76.9 \mathrm{Ci} / \mathrm{km}^{\mathrm{A}} 2$

$0.9 \operatorname{aCj} / \mathrm{k}^{\wedge} 2$ 


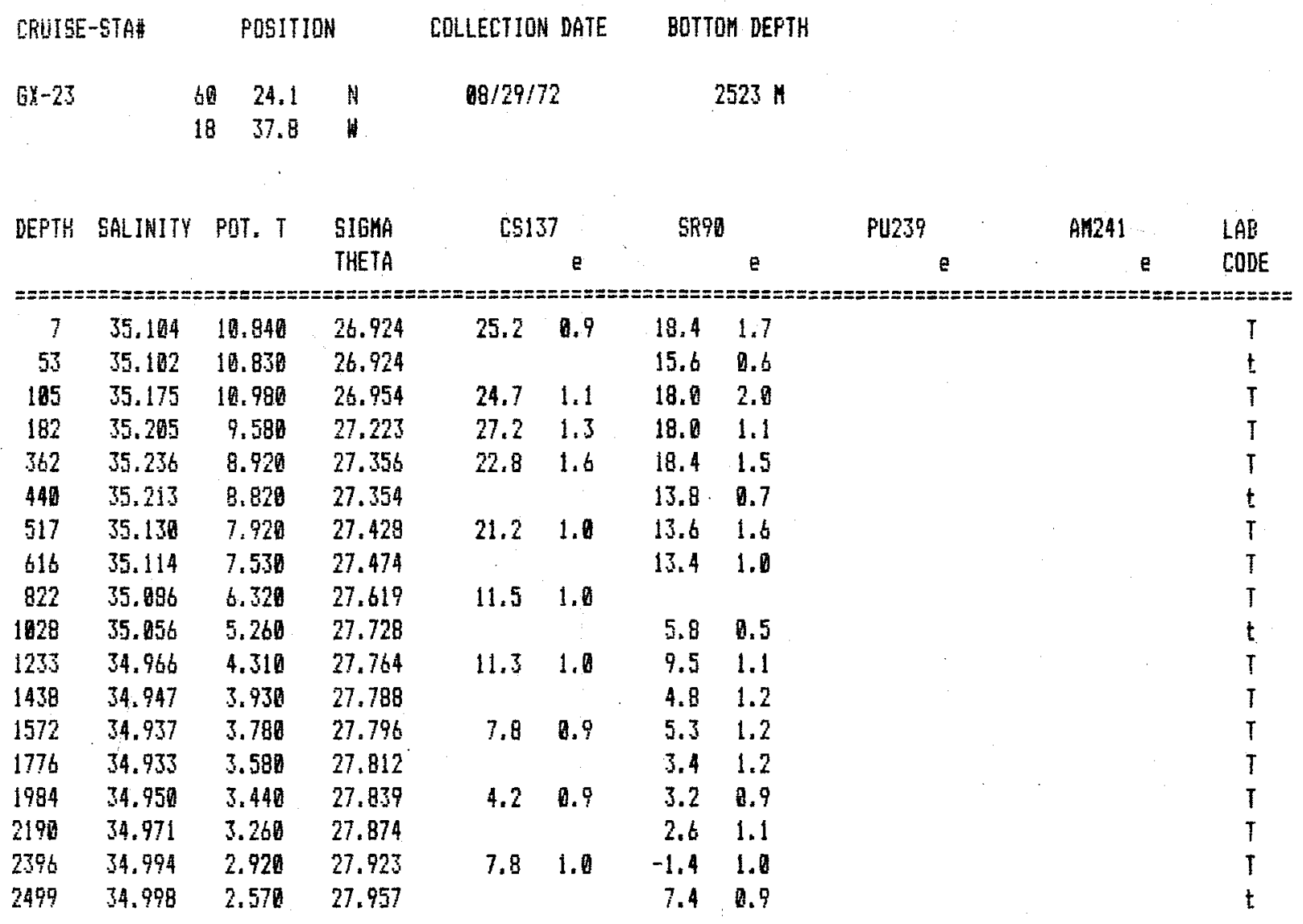

C5137 FPOH A $102523 \mathrm{M}=$ 5月90 From o TO $2523 \mathrm{M}=$
$141.3 \mathrm{mCi} / \mathrm{km}^{\wedge} 2$

$93.5 \mathrm{ACi} / \mathrm{k} \mathrm{m}^{\wedge} 2$ 


\begin{tabular}{|c|c|c|c|c|c|c|c|c|c|c|c|}
\hline \multicolumn{2}{|c|}{ CRUISE-STAI } & \multicolumn{2}{|c|}{ POSITION } & \multicolumn{2}{|c|}{ COLLECTION DATE } & \multicolumn{2}{|c|}{ BOTTOM DEPTH } & & & & \\
\hline \multirow[t]{3}{*}{$6 x-27$} & 4 & 1590 & $H$ & \multicolumn{2}{|c|}{$09 / 12 / 72$} & \multirow{2}{*}{\multicolumn{2}{|c|}{4875}} & & & & \\
\hline & 4 & 21.0 & $\omega$ & & & & & & & & \\
\hline & & & & & & & & & & & \\
\hline \multirow[t]{2}{*}{ DEFTH } & \multirow[t]{2}{*}{ SALINITY } & \multirow{2}{*}{ POT. T } & SIGKA & \multicolumn{2}{|c|}{$[5137$} & \multirow[t]{2}{*}{ SR9O } & & \multicolumn{2}{|c|}{ PU239 } & \multirow{2}{*}{ A月241 } & $\angle A B$ \\
\hline & & & THETA & & 8 & & e & & e & & CODE \\
\hline \multicolumn{12}{|c|}{ 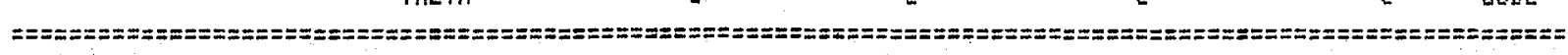 } \\
\hline 10 & 36.034 & & & 32.2 & 0.6 & 22.3 & 0.4 & 0.360 & 0. & & H \\
\hline 70 & 36.231 & & & & & 24.2 & 1.1 & & & & T \\
\hline 120 & 36.347 & & & 33.4 & 0.5 & 24.8 & 0.5 & 9.460 & 0.140 & & $W$ \\
\hline 225 & 36.679 & & & & & 19.3 & 1.2 & & & & T \\
\hline 260 & 35.967 & 14.980 & 26.752 & & & 21.6 & 1.6 & & & & $T$ \\
\hline 320 & 35.945 & & & 27.2 & 0.7 & 19.7 & 0.3 & & & & $W$ \\
\hline 490 & 35.715 & 13.640 & 26.848 & 23.1 & 0.4 & 14.4 & 0.4 & 0,410 & 0.880 & & H \\
\hline 595 & 35.518 & & & & & 14.2 & 0.8 & & & & $T$ \\
\hline 595 & 35.253 & 9.710 & 27.239 & 8.3 & 0.5 & 5.4 & 0.1 & 0.120 & 0.050 & & 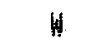 \\
\hline 735 & 34.889 & 9.000 & 27.971 & & & 7.1 & 0.9 & & & & $T$ \\
\hline 970 & 35.074 & 6.070 & 27.642 & 9.3 & 0.5 & 5.4 & 0.2 & 0.120 & 0.050 & & $\#$ \\
\hline 1096 & 35.840 & 5.180 & 27.725 & & & 7.7 & 0.8 & & & & T \\
\hline 1245 & 35.021 & 4.680 & 27.767 & 10.1 & 9.6 & 5.1 & 0.3 & 0.260 & 0.070 & & $H$ \\
\hline 1394 & 35.036 & 4.480 & 27.891 & & & 5.2 & 0.7 & & & & $T$ \\
\hline 1543 & 34.994 & 4.160 & 27.802 & 8.3 & 0.7 & 3.5 & 0.5 & 0.080 & 0,030 & & $H$ \\
\hline 1693 & 34.992 & 3.990 & 27.818 & & & 3.5 & 1.2 & & & & $T$ \\
\hline 1893 & 34.981 & 3.776 & 27.832 & 3.0 & 0.7 & 1.5 & 0.2 & 0.110 & 0.020 & & 11 \\
\hline 2543 & 34.958 & 3.230 & 27.866 & & & 1.8 & 1.1 & & & & 1 \\
\hline 3162 & 34.941 & 2.690 & 27.981 & 1.1 & 0.5 & 0.3 & 0.4 & 0.090 & & & $H$ \\
\hline 3778 & 34.919 & 2.190 & 27.925 & & & 0.3 & 1.0 & & & & $\mathrm{~T}$ \\
\hline 4396 & 34.911 & 1.890 & 27.934 & 8.9 & 0.7 & 8.8 & 0.5 & 8.099 & 0.030 & & H \\
\hline 4653 & 34.902 & 1.840 & 27.938 & & & 2.2 & 0.8 & & & & $T$ \\
\hline 4859 & 34,907 & 1.820 & 27.944 & 4.4 & a. 9 & 4.5 & 0.3 & D. 050 & 0.020 & & $W$ \\
\hline
\end{tabular}

CS137 FROM TO $4875 \mathrm{M}=141.0 \mathrm{aC \textrm {C }} / \mathrm{k}^{\wedge} 2$ SR96 FROH H TO $4875 \mathrm{~h}=98.5 \mathrm{mCj} / \mathrm{km}^{\mathrm{k} 2}$ FU239 FROM OA TO $4875 \mathrm{H}=3.06 \mathrm{aCj} / \mathrm{k} \mathrm{m}^{2} 2$ 


\begin{tabular}{|c|c|c|c|c|c|c|c|c|c|c|c|c|}
\hline \multicolumn{2}{|c|}{ LRUISE-STAZ } & \multicolumn{2}{|c|}{ POSITION } & \multicolumn{2}{|c|}{ COLLECTION DATE } & \multicolumn{2}{|c|}{ BOTTOM DEPTH } & & & & & \\
\hline \multirow[t]{2}{*}{$6 y-29$} & & $35 \quad 59.8$ & N & \multirow{2}{*}{\multicolumn{2}{|c|}{$69 / 17 / 72$}} & \multirow{2}{*}{\multicolumn{2}{|c|}{$4974 \mathrm{H}$}} & & & & & \\
\hline & & $47 \quad 1.1$ & H & & & & & & & & & \\
\hline \multirow{2}{*}{ DEPTH } & \multirow[t]{2}{*}{ SALINITY } & \multirow[t]{2}{*}{ FoT. T } & 5IGMA & \multicolumn{2}{|c|}{$[5137$} & \multicolumn{2}{|c|}{5890} & \multicolumn{2}{|c|}{ PU239 } & \multicolumn{2}{|c|}{ Al241 } & LAE \\
\hline & & & THETA & & e & & e & & e & & $\mathrm{e}$ & CODE \\
\hline \multicolumn{13}{|c|}{ 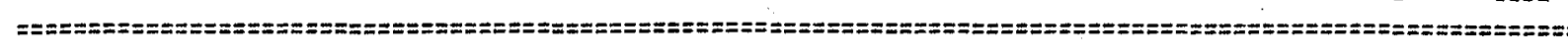 } \\
\hline 10 & 36.966 & & & 27.2 & 1.6 & 18.8 & 1.2 & 0.360 & 0.020 & 0.022 & 9.005 & 5 \\
\hline 31 & & & & 22.5 & 1.1 & 17.1 & 1.1 & & & & & 1 \\
\hline 70 & 36.424 & & & 27.6 & 0.8 & 24.0 & 1.5 & 0.210 & 0.010 & 0.924 & 0.005 & $\mathrm{~B}$ \\
\hline 140 & 36.524 & & & 35.0 & 1.9 & 21.8 & 1.6 & 0.300 & 0.020 & 0.043 & 9. 980 & 5 \\
\hline 190 & 36.449 & & & & & 22.8 & 1.8 & 0,330 & 0.020 & & & $\mathrm{R}$ \\
\hline 250 & 36.434 & & & 37.0 & 1.7 & 26.2 & 0.9 & 0.380 & 0.020 & & & $S$ \\
\hline 373 & 36.251 & & & 36.9 & 0.4 & $21.7^{\circ}$ & 0.8 & 0.300 & 9.040 & 0.940 & 0.030 & $\psi$ \\
\hline 440 & & & & 23.6 & 0.4 & 16.1 & 0.4 & 9.320 & 0.030 & & & i \\
\hline 469 & 36.995 & 15.530 & 26.725 & 26.6 & 1.6 & 21.4 & 0.8 & & & & & $T$ \\
\hline 554 & 35.862 & 14.190 & 26.843 & 20.1 & 8.4 & 13.2 & 0.8 & 0.289 & 0.930 & 1. 080 & 0.020 & v \\
\hline 639 & 35.696 & 12.940 & 26.979 & 17.4 & 0.3 & 13.4 & 1.1 & 0.240 & 0.020 & & & v \\
\hline 723 & 35.490 & 11.450 & 27.111 & & & 9.2 & 0.8 & & & & & $T$ \\
\hline 787 & 35.217 & 9.390 & 27.265 & 5.9 & 0.3 & 4.4 & 0.4 & 0.090 & 0.810 & & & $w$ \\
\hline 905 & 35.968 & & & 4.9 & 0.3 & 4.3 & 0.8 & 0.110 & 0.020 & & & y \\
\hline 1052 & 35.110 & 5.546 & 27.658 & 12.2 & 1.1 & 8.1 & 1.1 & 0.100 & 0.010 & & & 1 \\
\hline 1346 & 35.001 & 4.440 & 27.777 & 11.1 & 0.9 & 7.6 & 0.8 & & & & & 1 \\
\hline 1537 & 34.991 & 4.020 & 27.814 & 6.2 & 0.4 & 3.5 & 0.8 & 9.080 & 0.010 & & & $I$ \\
\hline 1925 & & & & 2.0 & 0.4 & 1.4 & 0.9 & 0.110 & 0.030 & & & 2 \\
\hline 2123 & 34.972 & 3.540 & 27.848 & 1.4 & 0.2 & 1.4 & 1.0 & & & & & !y \\
\hline 2455 & 34.959 & 3.000 & 27.888 & 9.9 & & -0.3 & 0.7 & 9.029 & 0. 2004 & & & 2 \\
\hline 3182 & 34.938 & 2.500 & 27.914 & 1.0 & 0.9 & 4.0 & 1.1 & 0.040 & 0.006 & & & $\bar{\theta}$ \\
\hline 3712 & 34.908 & 2.040 & 27.928 & 0.9 & 0.3 & 9.5 & 0.8 & 0.060 & 0.007 & & & 1 \\
\hline 4241 & 34.898 & 1.880 & 27.933 & 0.8 & 0.9 & 1.4 & 0.9 & 0.070 & 0.009 & & & $Q$ \\
\hline 4639 & 34.905 & & & 3.6 & 0.5 & 0.6 & 0.8 & 0.050 & Q. 006 & & & 2 \\
\hline
\end{tabular}

CSIS7 FFOM OA TO $3000 \mathrm{M}=$ SROQ FFOM O TO $3900 \mathrm{M}=$ FU23O FFOH O $104974 \mathrm{H}=$
$138.7 \mathrm{Ci} / \mathrm{km}^{\wedge} 2$

$96.1 \mathrm{mCi} / \mathrm{km}^{\mathrm{*}} 2$

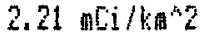




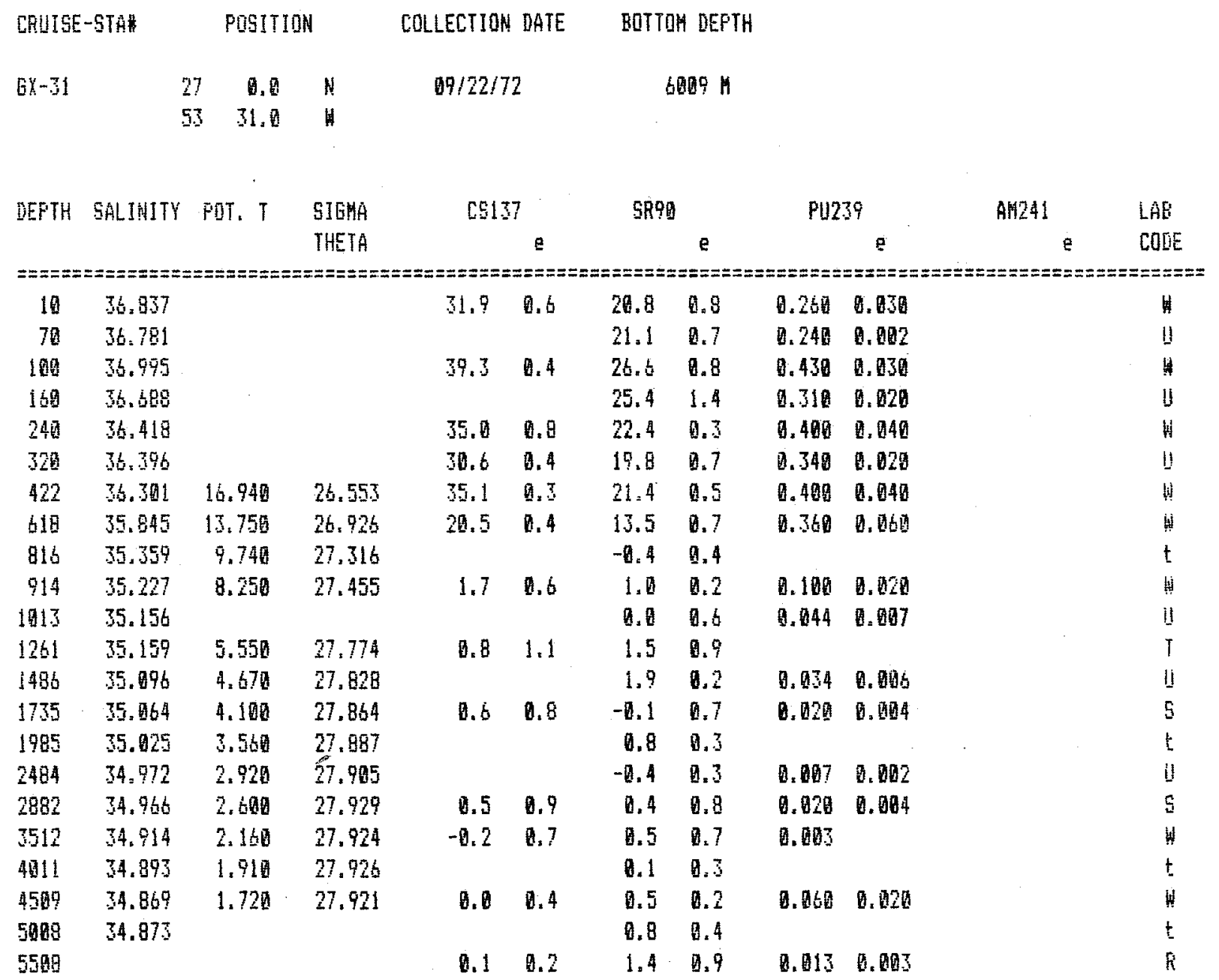

CS137 FROH H TO $1500 \mathrm{H}=180.4 \mathrm{mCj} / \mathrm{km}^{\wedge} 2$ SFPO FFOM H TO $1500 \mathrm{H}=$ PU23? FFOM I I TO $6099 \mathrm{M}=$
67.1 ori ikn's

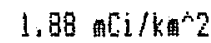




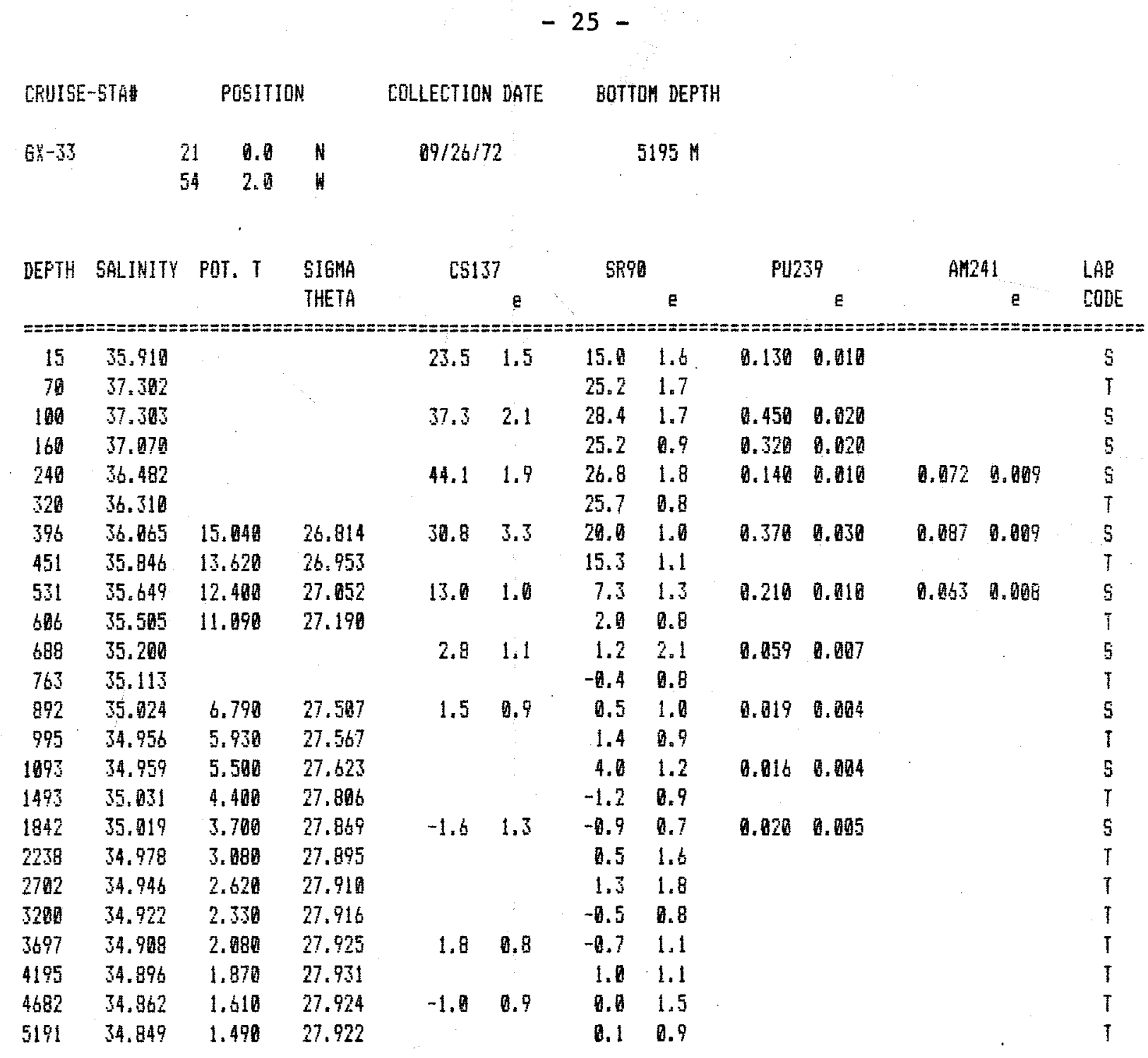

CS137 FROM B $\mathrm{H}$ TO $1900 \mathrm{H}=$ SF90 FROH O TD $1000 \mathrm{M}=$ PU239 FROA of $105195 \mathrm{H}=$
$86.8 \mathrm{Ci} / \mathrm{km} 2$

$54.9 \mathrm{ari} / \mathrm{km}^{*} 2$

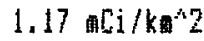




\begin{tabular}{|c|c|c|c|c|c|c|c|c|c|c|c|c|}
\hline \multicolumn{2}{|c|}{ CEUISE-STA } & \multicolumn{2}{|c|}{ POSITION } & \multicolumn{2}{|c|}{ COLLECTION DATE } & \multicolumn{2}{|c|}{ BOTTO\& DEPTH } & & & & & \\
\hline \multirow[t]{2}{*}{$6 x-37$} & & 1.5 & N & \multicolumn{2}{|c|}{$10 / 13 / 72$} & \multicolumn{2}{|c|}{$5064 \mathrm{H}$} & & & & & \\
\hline & & 0.8 & W & & & & & & & & & \\
\hline \multirow[t]{2}{*}{ DEPTH } & \multirow[t]{2}{*}{ SALINITY } & \multirow[t]{2}{*}{ POT. T } & SIGMA & \multicolumn{2}{|c|}{$[5137$} & \multirow[t]{2}{*}{ SR9D } & & \multicolumn{2}{|c|}{ PU239 } & \multicolumn{2}{|c|}{ AM 241} & LAB \\
\hline & & & THETA & & e & & e & & e & & e & CODE \\
\hline \multicolumn{13}{|c|}{ 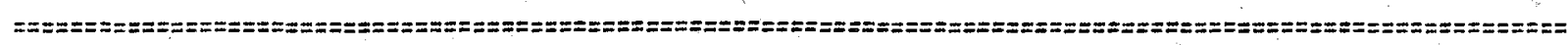 } \\
\hline 5 & 35.984 & & & 27.5 & 1.3 & 11.9 & 1.4 & 0.067 & 0.608 & & & $\mathrm{~g}$ \\
\hline 75 & 36.698 & & & 19.6 & 0.4 & 10.7 & 0.3 & 0.050 & 0.010 & & & H \\
\hline 191 & 36.780 & 22.130 & 25.550 & 26.5 & 0.4 & 16.8 & 2.2 & 0.092 & 0.013 & 0.021 & Q. & p \\
\hline 162 & 36.349 & 17.440 & 26.466 & 32.0 & 0.4 & 18.3 & 0.4 & 0.279 & 0.930 & & & $H$ \\
\hline 306 & 35.168 & 10.720 & 26.995 & 7.3 & 0.9 & 3.0 & 0.9 & & & & & $T$ \\
\hline 408 & 34.993 & 9.266 & 27.102 & 2.4 & 0.4 & 2.3 & 0.4 & 0.080 & 0.020 & & & W \\
\hline 510 & 34.778 & & & & & $1.0^{\circ}$ & 2.9 & & & & & $T$ \\
\hline 712 & 34.661 & 6.110 & 27.310 & -0.1 & 0.3 & -8.6 & 9.5 & 0.006 & 0.002 & & & $H$ \\
\hline 993 & 34.724 & 5.160 & 27.491 & 0.0 & 0.4 & 0.1 & 0.3 & 0.007 & & 0.010 & 0.007 & 4 \\
\hline 1094 & 34.815 & 4.980 & 27.569 & 8.8 & 0.9 & 1.3 & 0.9 & & & & & $T$ \\
\hline 1699 & 34.980 & & & & & 0.8 & 1.5 & & & & & $T$ \\
\hline 1983 & 34.964 & 3.300 & 27.883 & 0.4 & 0.4 & 0.2 & 0.5 & 0.030 & 0.020 & & & $H$ \\
\hline 2441 & 34.950 & 2.850 & 27.894 & 0.7 & 9.8 & 0.9 & a. 0 & & & & & $T$ \\
\hline 3472 & 34.906 & 2.196 & 27.914 & & & 0.3 & 0.8 & & & & & I \\
\hline 3985 & 34.895 & & & -0.2 & 0.3 & -0.5 & 9.1 & & & & & $H$ \\
\hline 4499 & $34.87 ?$ & 1.760 & 27.921 & 0.5 & 0.9 & -0.4 & 1.1 & & & & & $T$ \\
\hline 5014 & $37.8 \mathrm{ge}$ & 1.179 & 27.910 & 1.5 & 0.2 & & & 0.016 & 0.006 & 0.002 & & 0 \\
\hline
\end{tabular}

C5137 FROH A TO TO $1000 \mathrm{~K}=$ SROA FEOH OH TO $1000 \mathrm{M}=$

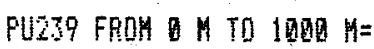

35.4 aci/km?

$19.7 \mathrm{aCi} / \mathrm{km}^{\wedge} 2$

$0.34 \mathrm{mCi} / \mathrm{k}^{\mathrm{A}} \mathrm{2} 2$ 


$\begin{array}{lrrccc}\text { CRUISE-STA } & & \text { POSITION } & \text { COLLECION DATE } & \text { BOTTON DEPTH } \\ \text { 6X-40 } & 3 & 30.0 & N & 10 / 19 / 72 & 4273 \mathrm{M} \\ & 38 & 0.0 & W & & \end{array}$

\begin{tabular}{|c|c|c|c|c|c|c|c|c|c|c|c|c|}
\hline DEPTH & SALINITY & PQT. T & $\begin{array}{l}\text { SIGHA } \\
\text { THETA }\end{array}$ & \multicolumn{2}{|c|}{$[5137$} & \multicolumn{2}{|c|}{$5 \% 90$} & \multicolumn{2}{|c|}{ PU239 } & \multicolumn{2}{|c|}{ AM241 } & $\begin{array}{l}\text { LAB } \\
\text { CODE }\end{array}$ \\
\hline \multicolumn{13}{|c|}{ 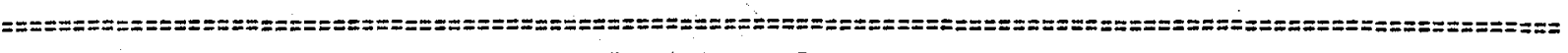 } \\
\hline 5 & 36.064 & & & 13.7 & 1.0 & 18.0 & 1.1 & 0.050 & 0.010 & 1. 099 & 0.603 & 5 \\
\hline 89 & 36.083 & & & & & 8.8 & 2.2 & 0.038 & 0.095 & & & E \\
\hline 100 & 36.151 & & & 18.2 & 1.1 & 14.5 & 1.3 & 0.945 & 0.005 & 1.005 & 9.904 & 5 \\
\hline 149 & 35.398 & 13,836 & $26.5 b 1$ & 8.9 & 0.5 & 5.7 & 0.8 & 0.070 & 0.010 & & & 7 \\
\hline 197 & 35.954 & 11.130 & 26.831 & 4.5 & 0.5 & 3.3 & 0.9 & 0.110 & 0.150 & & & I \\
\hline 296 & 34.896 & 9.810 & 26.942 & 5.1 & 0.9 & 1.9 & 0.9 & & & & & $T$ \\
\hline 392 & $34.77 ?$ & 8.500 & 27.963 & 2.0 & 0.4 & 0.2 & 0.8 & 0.040 & 0.005 & & & 2 \\
\hline 496 & 34.712 & & & 0.0 & 0.9 & -0.2 & 0.8 & 0.830 & 0.005 & & & 0 \\
\hline 685 & 34.547 & 5.450 & 27.394 & & & 9.4 & 0.9 & & & & & $T$ \\
\hline 790 & 34.554 & 4.830 & 27.379 & 0.8 & 0.9 & 0.2 & 1.0 & & & & & $T$ \\
\hline 889 & 34.694 & 4.6410 & 27.440 & 1.1 & 0.7 & 0.0 & 0.9 & 0.011 & 0.003 & & & $?$ \\
\hline 989 & 34.650 & 4.470 & 27.495 & 0.5 & 0.3 & -0.5 & 0.8 & 0.060 & 0.008 & & & 2 \\
\hline 1086 & 34.728 & 4.440 & 27,540 & & & 9.5 & 1.0 & & & & & $T$ \\
\hline 1386 & 34.927 & 4.300 & 27.733 & 0.9 & & 1.3 & 1.4 & -.010 & 0.010 & & & $I$ \\
\hline 1685 & 34.972 & & & 0.9 & & & & 0.020 & 0.006 & & & $L$ \\
\hline 1579 & 34.968 & 3.459 & 27.851 & 1.5 & 0.4 & 0.5 & 0.9 & 0.907 & 0.005 & & & 1 \\
\hline 2477 & 34.941 & 2.826 & 27.888 & 0.9 & & 0.5 & 1.0 & 0.007 & 0.002 & & & $I$ \\
\hline 2970 & 34.920 & 2.436 & 27.905 & 1.6 & 0.6 & -0.6 & 0.8 & 0.030 & 0.005 & & & 7 \\
\hline 3467 & 34.909 & & & 3,6 & 1.5 & 0.3 & 0.9 & -.097 & 0.002 & & & $I$ \\
\hline 3974 & 34.885 & 1.861 & 27.923 & 1.3 & 0.4 & 0.1 & 0.8 & 0.034 & 0.008 & & & $I$ \\
\hline 4179 & 34.828 & 1.361 & 27.914 & 1.1 & 0.3 & 2.4 & 1.4 & 0.016 & 0.607 & & & 7 \\
\hline
\end{tabular}

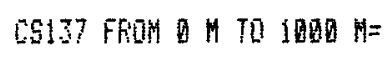
SR90 FRON O 11 TO $1000 \mathrm{H}=$ FU23\% FROA O $\mathrm{N}$ TO $1000 \mathrm{H}=$
$17.1 \mathrm{mCi} / \mathrm{kn}^{2} 2$

$0.5 \operatorname{aCi} / \mathrm{kn}^{\wedge} 2$

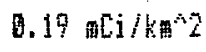




\begin{tabular}{|c|c|c|c|c|c|c|c|c|c|}
\hline \multicolumn{2}{|c|}{ CRUISE-STAZ } & \multicolumn{2}{|c|}{ POSITION } & \multirow{2}{*}{$\begin{array}{l}\text { COLLECTION DATE } \\
10 / 26 / 72\end{array}$} & \multirow{2}{*}{\multicolumn{2}{|c|}{$\begin{array}{c}\text { BOTTOH DEPTH } \\
5073\end{array}$}} & & & \\
\hline 6x-81日 & & $4 \quad 59.0$ & 5 & & & & & & \\
\hline & & 280.0 & H & & & & & & \\
\hline \multirow[t]{2}{*}{ DEPTH } & SALIHITY & FOT. T & SIGMA & {$[5137$} & SR9I & & PU239 & AM241 & LAB \\
\hline & & & THETA & e & & e & e & e & CODE \\
\hline \multicolumn{10}{|c|}{ 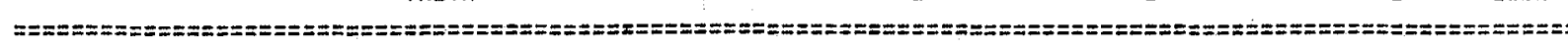 } \\
\hline 70 & 36.722 & & & & 9.8 & 0.9 & & & $T$ \\
\hline 160 & 35.237 & & & & 5.6 & 0.9 & & & $T$ \\
\hline 195 & 35.949 & 11.211 & 26.812 & 3.10 .4 & 1.8 & 0.3 & 0.2800 .040 & & 世 \\
\hline 295 & 34.781 & 8.940 & 26.996 & & 0.6 & 1.1 & & & $T$ \\
\hline$\$ 92$ & 34,538 & & & & -1.8 & 1.0 & & & $T$ \\
\hline 840 & 34.495 & 4.219 & 27.400 & & 0.6 & 1.2 & & & $T$ \\
\hline 107解 & 34.683 & 4.160 & 27.554 & & 1.2 & 8.9 & & & $T$ \\
\hline 1804 & 34.759 & 3.587 & 27.832 & & 1.1 & 1.1 & & & $T$ \\
\hline 2652 & 34.922 & 2.662 & 27.880 & & 0.9 & 0.9 & & & $T$ \\
\hline 4101 & 34.797 & 1.200 & 27.899 & & 1.9 & 1.8 & & & $T$ \\
\hline 5031 & 34.692 & 0.210 & 27.874 & & 1.3 & 1.0 & & & $T$ \\
\hline
\end{tabular}

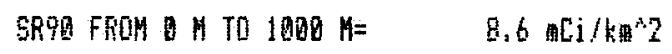




\begin{tabular}{|c|c|c|c|c|c|c|c|c|c|c|c|}
\hline \multicolumn{2}{|c|}{ CRUISE-STA } & \multicolumn{2}{|c|}{ POSITION } & \multirow{2}{*}{\multicolumn{2}{|c|}{$\begin{array}{l}\text { COLLECTION DATE } \\
10 / 29 / 72\end{array}$}} & \multirow{2}{*}{\multicolumn{2}{|c|}{$\begin{array}{c}\text { BOTTON DEPTH } \\
5581 \mathrm{M}\end{array}$}} & & & & \\
\hline $6 x-49$ & 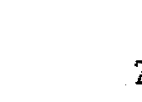 & $\begin{array}{rr}7 & 12.6 \\
6 & 9.9\end{array}$ & $\begin{array}{l}5 \\
H\end{array}$ & & & & & & & & \\
\hline DEPTH & SALIHITY & POT. T & $\begin{array}{l}\text { SIGHA } \\
\text { THETA }\end{array}$ & \multicolumn{2}{|c|}{ C5137 } & \multicolumn{2}{|c|}{ SR90 } & \multicolumn{2}{|c|}{$F \cup 239$} & AM241 & $\begin{array}{l}\angle A B \\
C O D E\end{array}$ \\
\hline \multicolumn{12}{|c|}{ 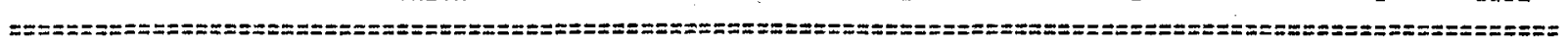 } \\
\hline 6 & 36.291 & 26.180 & 23.974 & 12.8 & 0.4 & 9.5 & 0.2 & 0.340 & 8. 030 & & W \\
\hline$b i$ & 36.272 & 26.060 & 24.006 & 13.6 & 0.9 & 8.1 & 0.9 & & & & $T$ \\
\hline 116 & 36.456 & 22.420 & 25.223 & 8.0 & 0.4 & 8.2 & 0.3 & 0.248 & 0.020 & & 诵 \\
\hline 156 & 35.741 & 16.410 & 26.248 & 6.7 & 1.1 & 5.4 & 0.9 & & & & $T$ \\
\hline 255 & 35.033 & & & -9.8 & 0.2 & 0.0 & 0.3 & 0.040 & 0.020 & & $H$ \\
\hline 375 & 34.603 & 7.123 & 27.130 & -8.5 & 1.0 & 1.5 & 0.9 & & & & $T$ \\
\hline 445 & 34.584 & & & 0.2 & 0.2 & & & 0.010 & 0.820 & & $W$ \\
\hline 598 & 34.599 & 5.612 & 27.253 & -0.3 & 0.9 & 0.1 & 0.8 & & & & $T$ \\
\hline 742 & 34.469 & & & 1.2 & 0.2 & & & 0.010 & 0.006 & & H \\
\hline 844 & 34.479 & 4.350 & 27.372 & -1.0 & 0.7 & 0.2 & 0.9 & & & & $T$ \\
\hline 1245 & 34.774 & 4.040 & 27.630 & -0.7 & 1.1 & 0.2 & 0.8 & & & & $T$ \\
\hline 1292 & 34.899 & 4.050 & 27.666 & 1.7 & 0.3 & 1.5 & 0.4 & 0.030 & 0.070 & & W \\
\hline 1688 & 34.959 & 3.670 & 27.824 & -0.8 & 1.1 & -0.5 & 0.9 & & & & $T$ \\
\hline 2087 & 34.94 .3 & 3.070 & 27.869 & -0.2 & 0.2 & 0.5 & 0.3 & 0.000 & 0.010 & & $W$ \\
\hline 2370 & 34.920 & & & 0.1 & 0.9 & 0.1 & 0.4 & & & & $T$ \\
\hline 3363 & 34.907 & 2.270 & 27.908 & -1.0 & 0.9 & -0.3 & 0.8 & & & & $T$ \\
\hline 4457 & 34.743 & 0.610 & $27.89 \%$ & -0.8 & 1.2 & 0.7 & 0.9 & & & & $T$ \\
\hline 4995 & 34.722 & 0.296 & 27,894 & 0.3 & 1.5 & -0.1 & 0.8 & & & & $T$ \\
\hline
\end{tabular}

C5137 FRON 4 TO $590 \mathrm{H}=$ SRPG FFOH O TO $500 \mathrm{~N}=$ PU239 FroM o TO 590 H=
$9.4 \mathrm{mCi} / \mathrm{kar}^{2}$
8.2 aci $/ \mathrm{kA}+2$
$0.26 \mathrm{mCi} / \mathrm{K}^{\wedge} 2$ 


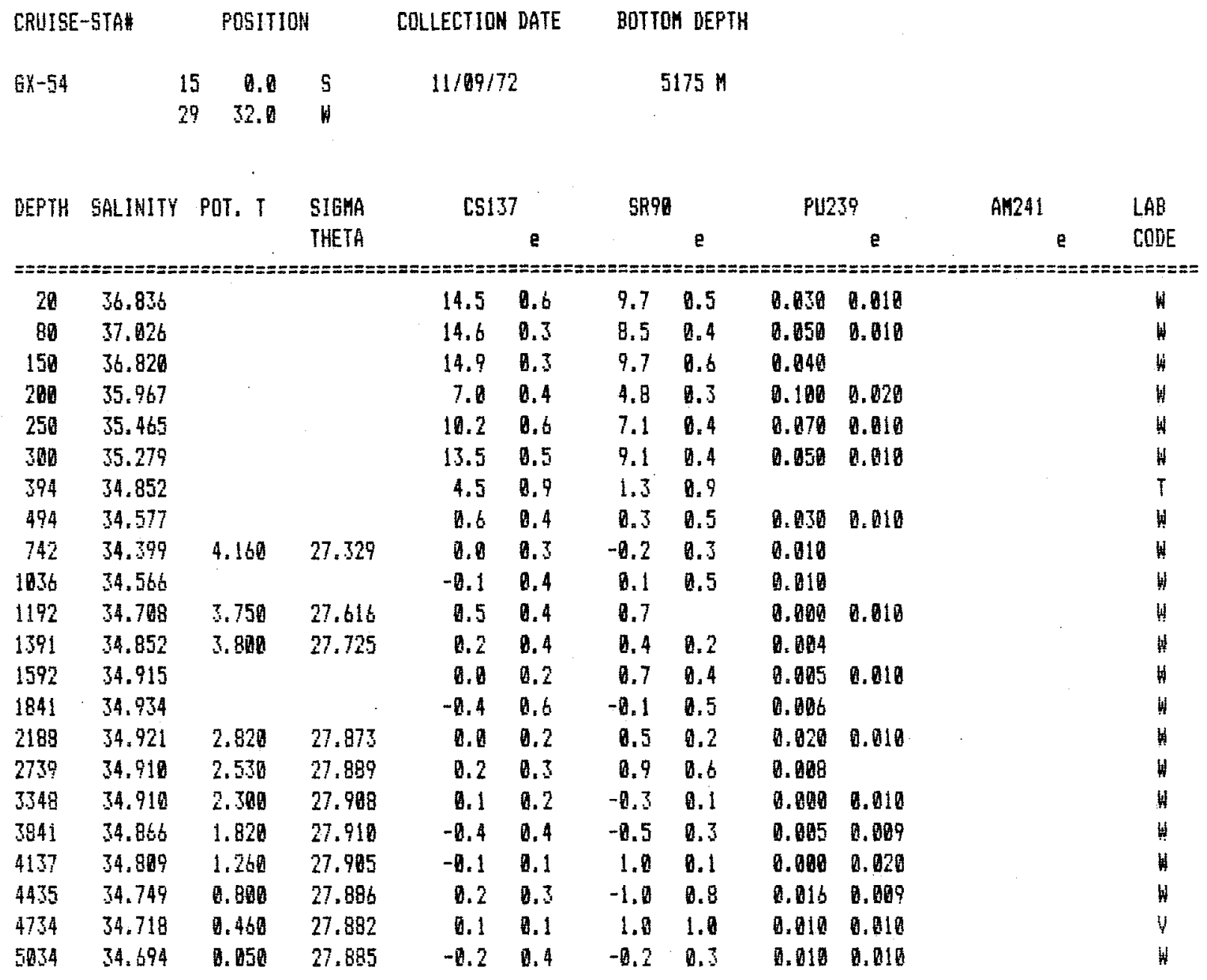

CS137 FROM O $\mathrm{HO}$ TO $1000 \mathrm{H=}$

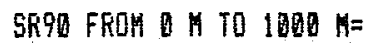

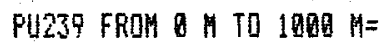

$22,3 \mathrm{BC \textrm {Ci }} / \mathrm{k} \mathrm{m}^{\mathrm{*}}-2$

13.8 解 $/ \mathrm{k}$ 田2

0. $15 \mathrm{mCi} / \mathrm{k}^{2} 2$ 


\begin{tabular}{|c|c|c|c|c|c|c|c|c|c|c|c|}
\hline \multicolumn{2}{|c|}{ CRUISE-STA\# } & \multicolumn{2}{|c|}{ POSITION } & \multicolumn{2}{|c|}{ COLLECTION DATE } & \multicolumn{2}{|c|}{ BOTTOM DEPTH } & & & & \\
\hline \multirow[t]{2}{*}{ 6y-56 } & & 1.5 & $\mathrm{~s}$ & \multicolumn{2}{|c|}{$11 / 13 / 72$} & \multirow{2}{*}{\multicolumn{2}{|c|}{$4313 \mathrm{M}$}} & & & & \\
\hline & & 0.0 & $H$ & & & & & & & & \\
\hline \multirow[t]{2}{*}{ DEPTH } & \multirow[t]{2}{*}{ SALINITY } & \multirow[t]{2}{*}{ POT. T } & SIGHA & \multirow{2}{*}{\multicolumn{2}{|c|}{ CS137 }} & \multirow[t]{2}{*}{589} & & \multicolumn{2}{|c|}{ PU239 } & AM241 & LAB \\
\hline & & & THETA & & & & e & & e & e & CODE \\
\hline \multicolumn{12}{|c|}{ 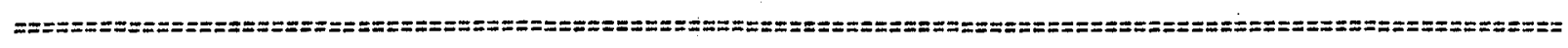 } \\
\hline 25 & 37.868 & & & 18.5 & 0.4 & 11.8 & 0.9 & 0.040 & 0.010 & & $W$ \\
\hline 99 & 37.075 & & & & & 12.4 & 0.9 & & & & $T$ \\
\hline 110 & 36.996 & & & 20.9 & 0.4 & 16.0 & 0.5 & & & & $H$ \\
\hline 230 & 35.667 & & & & & 13.9 & 1.0 & & & & $T$ \\
\hline 299 & 35.339 & & & 15.7 & 0.3 & 9.4 & 0.3 & 0. 880 & 0.020 & & 4 \\
\hline 418 & 34.979 & & & & & 3.9 & 0.9 & & & & $\mathrm{~T}$ \\
\hline 517 & 34.795 & & & 3.3 & 0.5 & 1.9 & 0.1 & 0.050 & 0.026 & & W \\
\hline 687 & 34.408 & 5.160 & 27.226 & & & 0.6 & 0.5 & & & & $T$ \\
\hline 997 & 34.470 & 3.368 & 27.464 & 0.0 & 0.3 & 0.2 & 8.4 & 0.020 & 0.610 & & 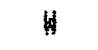 \\
\hline 1687 & 34.546 & 3.413 & 27.536 & & & 1.1 & 1.7 & & & & $T$ \\
\hline 1283 & 34.78 .3 & & & & & -1.1 & 0.9 & & & & 1 \\
\hline 1474 & 34.931 & 3.890 & 27.779 & 0.4 & 0.3 & -8.2 & 0.3 & 0.020 & 0.010 & & H \\
\hline 1874 & 34.956 & & & & & -0.4 & 1.1 & & & & $\mathrm{~T}$ \\
\hline 2370 & 34.937 & 2.880 & 27.880 & & & -0.5 & 0.8 & & & & $T$ \\
\hline 3340 & 34.992 & & & & & 0.4 & 0.9 & & & & $T$ \\
\hline 3486 & 34.873 & 1.910 & 27.909 & -0.1 & 0.4 & -0.2 & 0.6 & B. 804 & & & $W$ \\
\hline 3733 & 34.811 & & & & & -0.1 & 0.7 & & & & $T$ \\
\hline 3977 & 34.767 & 0.900 & 27.895 & 0.5 & 0.3 & -0.1 & 0.5 & & & & $H$ \\
\hline
\end{tabular}




$\begin{array}{lccccc}\text { CRUISE-STA\# } & & \text { POSITION } & \text { COLLECTION DATE } & \text { BOTTOH DEPTH } \\ 6 \times-58 & 27 & 0.0 & 5 & 11 / 18 / 72 & 4586 \mathrm{M} \\ & 37 & 0.0 & \$ & & \end{array}$

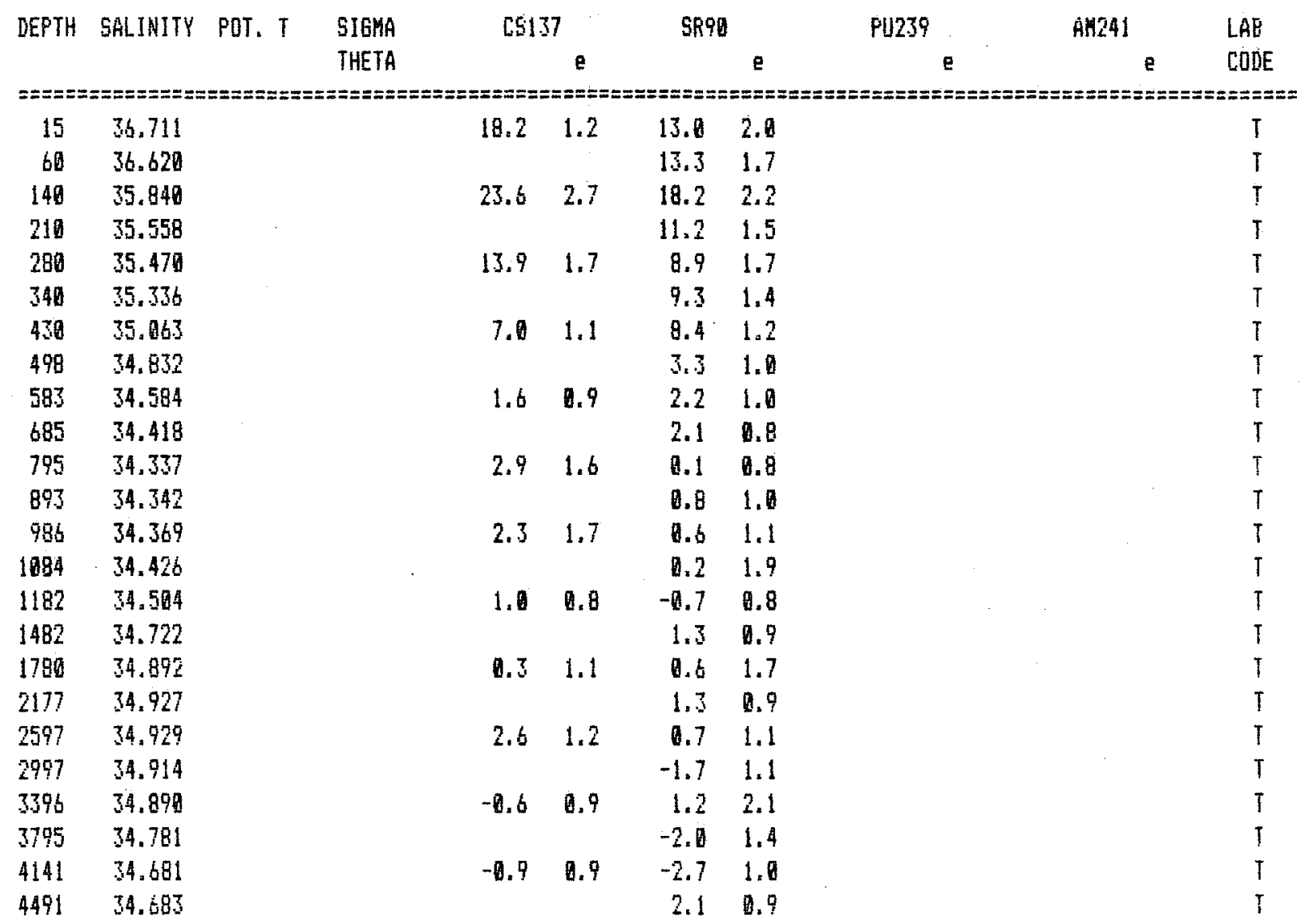




\begin{tabular}{|c|c|c|c|c|c|c|c|c|c|c|c|}
\hline \multicolumn{2}{|c|}{ CRUISE-STA } & \multicolumn{2}{|c|}{ POSITIDN } & \multicolumn{2}{|c|}{ COLLECTION DATE } & \multicolumn{2}{|c|}{ EOTTOM DEPTH } & & & & \\
\hline \multirow[t]{2}{*}{$6 x-60$} & \multirow{2}{*}{\multicolumn{2}{|c|}{$\begin{array}{ll}32 & 58.8 \\
42 & 31.8\end{array}$}} & 5 & \multicolumn{2}{|c|}{$11 / 22 / 72$} & \multicolumn{2}{|c|}{$4481 n$} & \multirow{2}{*}{\multicolumn{2}{|c|}{$\therefore \quad \therefore$}} & & \\
\hline & & & $H$ & & & 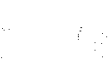 & & & & \\
\hline \multirow[t]{2}{*}{ DEPTH } & \multirow[t]{2}{*}{ SALINITY } & \multirow[t]{2}{*}{ POT. T } & SIGMA & \multicolumn{2}{|c|}{$\operatorname{cs137}$} & \multicolumn{2}{|c|}{$5 R 90$} & \multicolumn{2}{|c|}{ PU23? } & AM241 & LAB \\
\hline & & & THETA & & e & & e & & e & $\mathrm{E}$ & CODE \\
\hline \multicolumn{12}{|c|}{ 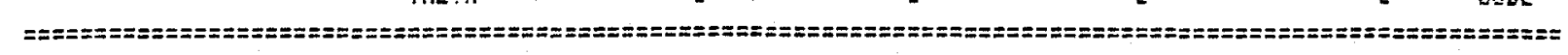 } \\
\hline 20 & 36.086 & & & 19.6 & 0.5 & 13.4 & 0.5 & 0.090 & 0.030 & & $w$ \\
\hline 60 & 35.979 & & & & & 14.8 & 1.3 & & & & $T$ \\
\hline 150 & 35.764 & & & 17.0 & 0.6 & 12.1 & 0.4 & 0.090 & 0.030 & & 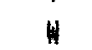 \\
\hline 220 & 35.622 & & & 13.5 & 0.6 & 9.6 & 0.9 & 0. 089 & 0.020 & & 7 \\
\hline 300 & 35.581 & & & 13.6 & 0.4 & 9.2 & 0.3 & 0.090 & 0.020 & & 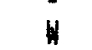 \\
\hline 350 & 35.407 & & & & & 8.4 & 1.2 & & & & $\mathrm{~T}$ \\
\hline 412 & 35.235 & & & 10.0 & 0.5 & 6.2 & 0.3 & 0.090 & 0.020 & & H \\
\hline 493 & 34.994 & & & 5.3 & 0.6 & -8.4 & 0.7 & 0.120 & 0.015 & & 2 \\
\hline 593 & 34.577 & & & 4.0 & 0.4 & 2.5 & 0.1 & 0.060 & 0.010 & & 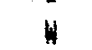 \\
\hline 693 & 34.341 & 5.730 & 27.105 & & & 2.4 & 0.3 & & & & i \\
\hline 793 & 34.299 & 4.880 & 27.172 & 2.2 & 0.4 & 1.4 & 0.4 & 0.030 & & & H \\
\hline 992 & 34.269 & 3.660 & 27.275 & & & 0.6 & 1.8 & 0.018 & 0.005 & & $i$ \\
\hline 1189 & 34.342 & 2.970 & 27.398 & 0.6 & 0.4 & -0.1 & 0.1 & 0.036 & 0.010 & & 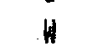 \\
\hline 1386 & 34.468 & 2.720 & 27.520 & 0.5 & 0.4 & 0.2 & 0.8 & 0.011 & 0.005 & & $i$ \\
\hline 1783 & 34.696 & & & 0.2 & 0.3 & -0.7 & 0.6 & 0.005 & & $0.007 \quad 0.004$ & i \\
\hline 2181 & 34.868 & 2.980 & 27.816 & & & -0.6 & 1.1 & 0.007 & 0.007 & & 7 \\
\hline 2584 & 34.917 & 2.890 & 27.864 & 0.1 & 0.6 & 0.2 & 0.2 & 0.020 & & & in \\
\hline 3167 & 34.912 & 2.430 & 27.899 & 1.1 & & & & 0.016 & 0.003 & & $\ddot{L}$ \\
\hline 3464 & 34.838 & 1.740 & 27.894 & -0.5 & 0.6 & 0.6 & 0.2 & 0.005 & & & ii \\
\hline 3762 & 34.741 & 0.710 & 27.686 & & & -0.1 & 0.8 & 0.005 & 0.802 & & $z$ \\
\hline 396.1 & 34.689 & & & 0.3 & 0.4 & 0.9 & 0.2 & 0.810 & & & $W$ \\
\hline 4159 & 34.676 & -0.150 & 27.880 & 0.1 & 0.1 & 0.5 & 0.8 & & & & I \\
\hline 4358 & 34.681 & -8.220 & 27.888 & -8.6 & 0.4 & 0.1 & 0.2 & 0.010 & & & W \\
\hline
\end{tabular}

C5137 FROH O $\mathrm{N}$ TO $1090 \mathrm{M}=$ SR90 FROM O if TO $1000 \mathrm{H}=$ PU239 FFOM O 4 TO $4401 . N=$
37.8 m $\mathrm{Ai} / \mathrm{kn} \mathrm{An}^{\wedge}$

24.? $\mathrm{Ci} / \mathrm{kg}^{\wedge} 2$

0. $48 \mathrm{mCi} / \mathrm{km}^{2} 2$ 


\begin{tabular}{|c|c|c|c|c|c|c|c|c|c|c|}
\hline \multicolumn{2}{|c|}{ CFUISE-STA\# } & \multicolumn{2}{|c|}{ POSITION } & \multirow{2}{*}{\multicolumn{2}{|c|}{$\begin{array}{l}\text { COLLECTION DATE } \\
12 / 05 / 72\end{array}$}} & \multirow{2}{*}{\multicolumn{2}{|c|}{$\begin{array}{c}\text { BOTTOH DEPTH } \\
5340 \mathrm{M}\end{array}$}} & & & \\
\hline \multirow[t]{2}{*}{$6 x-64$} & 3 & 3.5 & 5 & & & & & & & \\
\hline & 4 & $18 \quad 33.0$ & $H$ & & & & & & & \\
\hline \multirow[t]{2}{*}{ DEPTH } & SALINITY & POT. T & SIGHA & $\operatorname{csi}$ & & $5 R 98$ & & PIJ239 & AN241 & $\angle A B$ \\
\hline & & & THETA & & e & & e & e & e & CODE \\
\hline \multicolumn{11}{|c|}{ 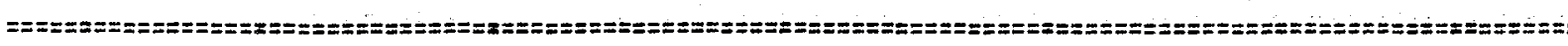 } \\
\hline 2 & 34.443 & 14.390 & 25.710 & 13.2 & 0.9 & 9.9 & 0.9 & & & $T$ \\
\hline 51 & 34.883 & 12.640 & 26.409 & & & 8.9 & 9.9 & & & $T$ \\
\hline 101 & 34.918 & 12.598 & 26.446 & 8.8 & 1.1 & 7.6 & 0.8 & & & $T$ \\
\hline 250 & 34.590 & & & & & 3.8 & 1.1 & & & T \\
\hline 397 & 34.226 & & & 3.7 & 1.0 & 1.6 & 8.8 & & & $T$ \\
\hline 594 & 34.197 & & & & & 1.7 & 0.9 & & & $T$ \\
\hline 793 & 34.246 & 3.120 & 27.308 & 1.5 & 0.9 & 0.0 & 8.8 & & & $T$ \\
\hline 1012 & $34.33 B$ & 2.760 & 27.413 & & & 1.5 & 0.8 & & & $T$ \\
\hline 1453 & 34.516 & & & 0.2 & 0.9 & 1.5 & 1.0 & & & $T$ \\
\hline 1575 & 34.586 & 2.460 & 27.636 & & & -0.3 & 0.8 & & & $T$ \\
\hline 1935 & 34.707 & & & -0.5 & 8.9 & -0.7 & 0.9 & & & $T$ \\
\hline 2475 & 34.840 & & & -0.6 & 1.0 & 1.7 & 0.8 & & & $T$ \\
\hline 2990 & 34.816 & & & & & -0.5 & 0.9 & & & $T$ \\
\hline 3460 & 34.743 & & & -0.9 & 0.9 & 0.9 & 0.9 & & & $T$ \\
\hline 3949 & 34.706 & & & -8.7 & 0.9 & 0.0 & a. 8 & & & $T$ \\
\hline$\$ 446$ & 34.678 & 0.040 & 27.872 & & & 8.7 & 1.1 & & & $T$ \\
\hline 5195 & 34,668 & & & -0.3 & 0.9 & 0.5 & 0.8 & & & $T$ \\
\hline
\end{tabular}




\begin{tabular}{|c|c|c|c|c|c|c|c|c|c|c|c|}
\hline \multicolumn{2}{|c|}{ CRUISE-STAR } & \multicolumn{2}{|c|}{ Position } & \multicolumn{2}{|c|}{ COLLECTION DATE } & \multicolumn{2}{|c|}{ BOTTOM DEPTH } & & & & \\
\hline $6 x-b 7$ & & $\begin{array}{ll}14 & 58.0 \\
11 & 10.0\end{array}$ & $\mathrm{~s}$ & \multicolumn{2}{|c|}{$12 / 10 / 72$} & \multicolumn{2}{|c|}{$5846 \mathrm{H}$} & & & & \\
\hline DEPTH & SALIHITY & POT. T & $\begin{array}{l}\text { SIGKA } \\
\text { THETA }\end{array}$ & & $\operatorname{cs} 137$ & \multicolumn{2}{|c|}{$5 R 90$} & \multicolumn{2}{|c|}{ PU239 } & AM241 & $\begin{array}{l}\text { LAB } \\
\text { CODE }\end{array}$ \\
\hline \multicolumn{12}{|c|}{ 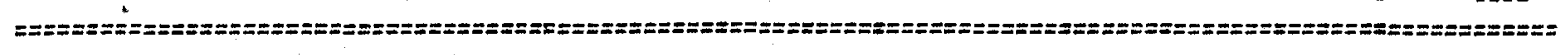 } \\
\hline 5 & 34.691 & 8.250 & 26.962 & 9.3 & 0.4 & 6.4 & 0.4 & 0.040 & 0.010 & & 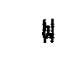 \\
\hline 95 & 34.675 & 8.898 & 26.922 & & & 8.9 & 1.1 & & & & $T$ \\
\hline 180 & 34.595 & 7.850 & 27.019 & 9.3 & 0.4 & 5.7 & 0.5 & 0.040 & 0.010 & & 4 \\
\hline 295 & 34.292 & 5.360 & 27.111 & & & 5.8 & 0.9 & & & & $T$ \\
\hline 391 & 34.196 & 4.448 & 27.139 & 4.9 & 0.3 & 4.4 & 0.2 & 0.050 & 0.020 & & $H$ \\
\hline 780 & 34.217 & 3.220 & 27.276 & 2.5 & 0.4 & 1.6 & 0.6 & 0.040 & 0.010 & & W \\
\hline 840 & 34.275 & & & 1.6 & 0.3 & 1.3 & 0.4 & 0.060 & 0.020 & & H \\
\hline 1022 & 34.334 & & & 1.2 & 0.3 & 0.5 & 0.4 & 0.020 & 0.010 & & 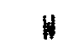 \\
\hline 1173 & 34.417 & 2.550 & 27.493 & & & 9.8 & 0.7 & & & & $T$ \\
\hline 1378 & 34.513 & 2.490 & 27.576 & 0.3 & 0.3 & 1.2 & 0.2 & 0.020 & 0.020 & & $n$ \\
\hline 1721 & 34.640 & 2.440 & 27.681 & & & -0.2 & 5.1 & & & & $T$ \\
\hline 1870 & 34.717 & 2.440 & 27.743 & -0.1 & 0.4 & -0.2 & 0.0 & 0.020 & 0.010 & & $W$ \\
\hline 2437 & 34.930 & 2.530 & 27.825 & & & 0.0 & 0.8 & & & & $T$ \\
\hline 3203 & 34.781 & 1.660 & 27.854 & & & 0.3 & 0.8 & & & & $T$ \\
\hline 4759 & 34.676 & 0.090 & 27.973 & & & 0.7 & 1.0 & & & & $T$ \\
\hline
\end{tabular}

C5137 FFOM O $\mathrm{NO} 1200 \mathrm{M}=$ SR90 FROM 120 TO $1200 \mathrm{H}=$ PU239 FROM II TO $1200 \mathrm{H}=$
22.8 配 $/ \mathrm{k}^{\mathrm{A}} \mathrm{T}^{2}$

$17.2 \mathrm{ACl} / \mathrm{km} 2$

0. $22 \mathrm{mC \textrm {Ci } / \mathrm { m }} \times 2$ 


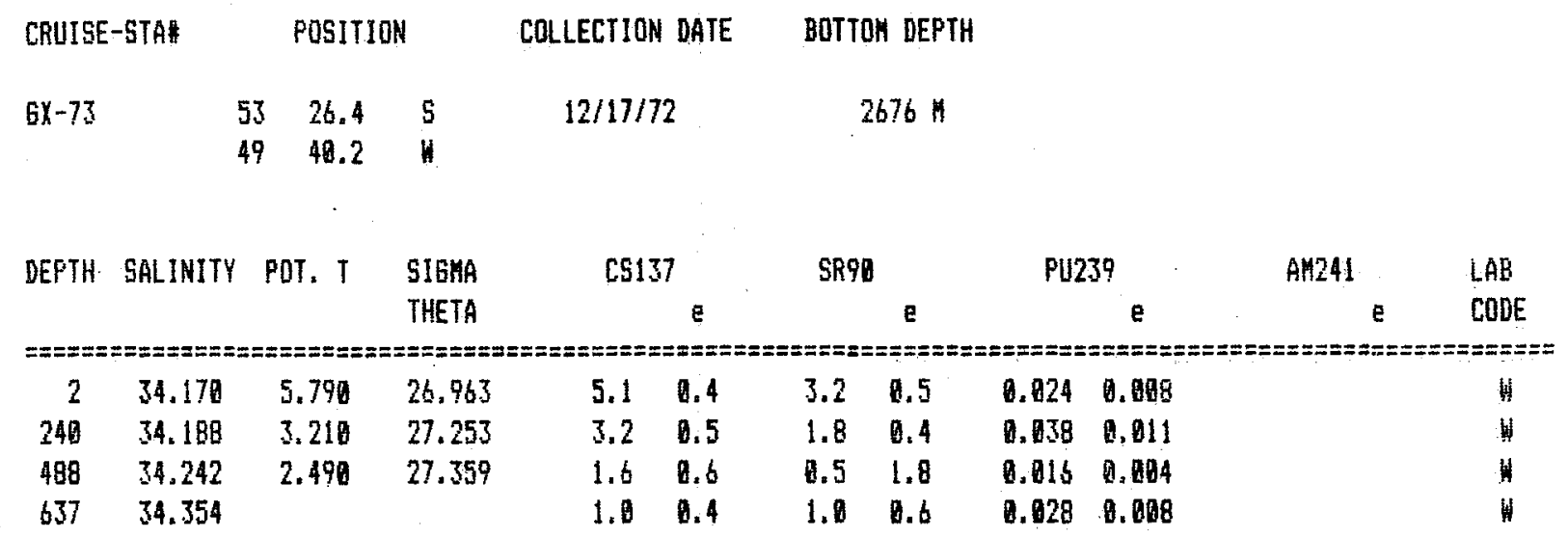

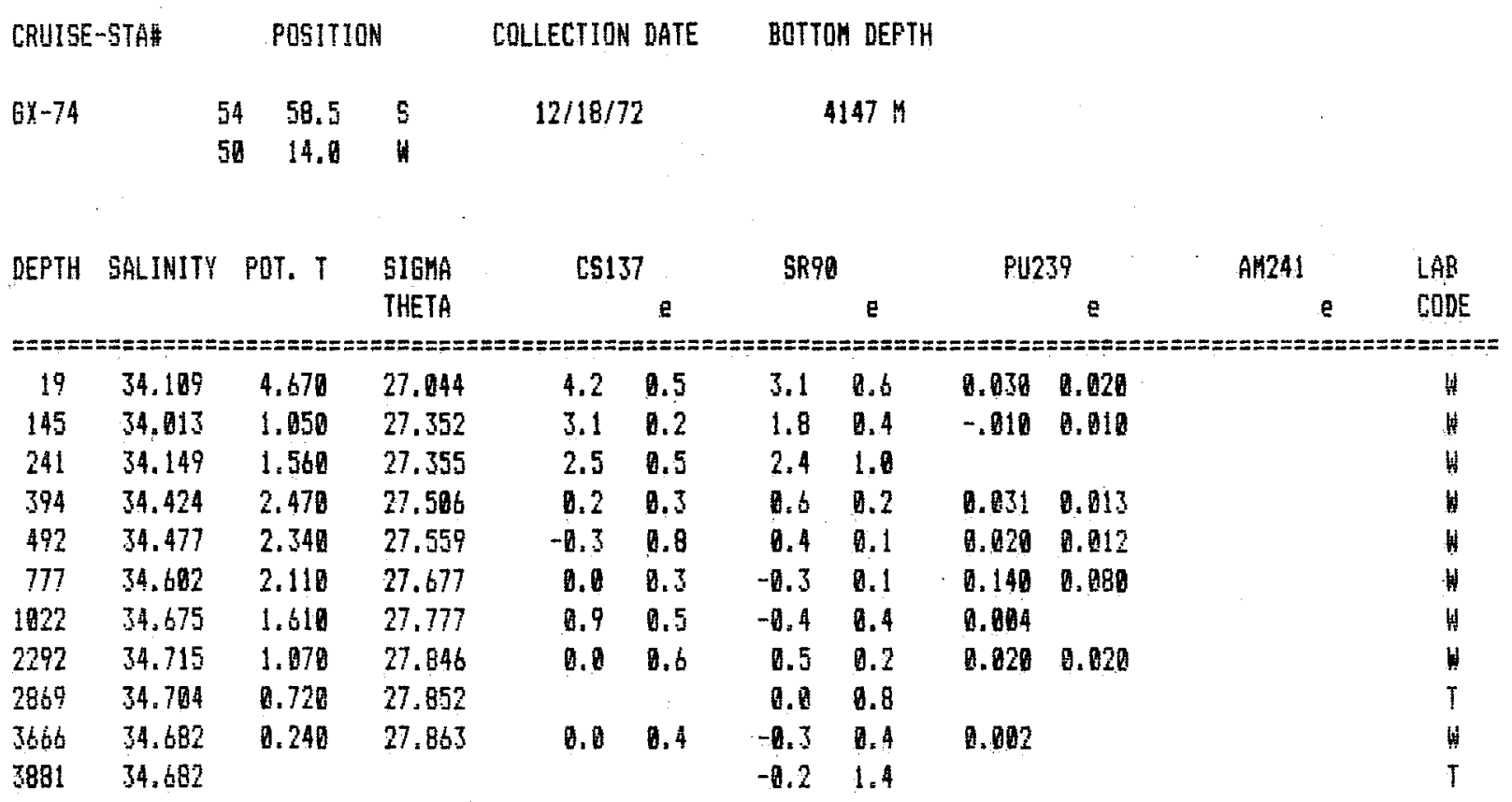

C5137 FROM O TO $500 \mathrm{H}=$ SRPQ FFin of to $580 \mathrm{H}=$
$4.6 \pi \operatorname{ci} / \mathrm{km}^{\wedge} 2$

$3.8 \mathrm{nCi} / \mathrm{kn}^{\mathrm{A}} 2$ 


\begin{tabular}{|c|c|c|c|c|c|c|c|c|c|c|c|}
\hline \multicolumn{2}{|c|}{ CRUISE-STAZ } & \multicolumn{2}{|c|}{ POSITION } & \multicolumn{2}{|c|}{ CQLLECTION DATE } & \multicolumn{2}{|c|}{ GOTTOM DEPTH } & & & & \\
\hline \multirow[t]{2}{*}{ GX-76 } & & 542.5 & 5 & \multicolumn{2}{|c|}{$01 / 01 / 73$} & \multirow{2}{*}{\multicolumn{2}{|c|}{$4682 \mathrm{M}$}} & & & & \\
\hline & 5 & 612.8 & W & & & & & & & & \\
\hline \multirow{2}{*}{ DEPTH } & \multirow[t]{2}{*}{ SALINITY } & \multirow[t]{2}{*}{ POT. T } & SIGMA & \multicolumn{2}{|c|}{$[5137$} & \multicolumn{2}{|c|}{ SA99 } & \multicolumn{2}{|c|}{ PU239 } & AM241 & LAE \\
\hline & & & THETA & & g & & e & & e & e & CONE \\
\hline \multicolumn{12}{|c|}{ 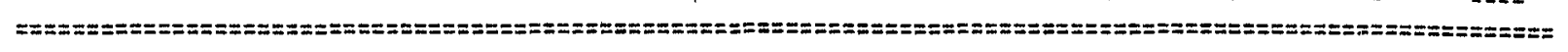 } \\
\hline 64 & 33.813 & 1.530 & 27.088 & 4.3 & 0.4 & 4.4 & 0.2 & 0.027 & 0.097 & & W \\
\hline 176 & 33.970 & -0.030 & 27.396 & 3.7 & 0.3 & 4.3 & 0.3 & 0.020 & 0.010 & & 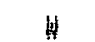 \\
\hline 252 & 34.053 & 0.990 & 27.316 & & & 2.9 & 0.8 & & & & $T$ \\
\hline 324 & 34.141 & 1.120 & 27.379 & 2.8 & 0.3 & 1.5 & 0.1 & 0,040 & 0.970 & & 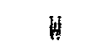 \\
\hline 400 & 34.236 & & & & & 1.1 & 0,8 & & & & T \\
\hline 493 & 34.309 & 1.860 & 27,462 & 0.8 & 0.4 & & & 0.020 & 0.005 & & $w$ \\
\hline 693 & 34.472 & & & & & 1.8 & 0.8 & & & & T \\
\hline 842 & 34.547 & 2.210 & 27.625 & 0.3 & 0.2 & 0.3 & 0.2 & 0.020 & 0.020 & & 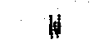 \\
\hline 988 & 34.602 & & & & & -0.7 & 0.9 & & & & T \\
\hline 1202 & 34.648 & 2.100 & 27.715 & 0.4 & 0.4 & & & & & & w \\
\hline 1401 & 34.684 & & & & & -0.6 & 0.9 & & & & $T$ \\
\hline 2090 & 34.722 & & & & & -0.3 & 0.8 & & & & $T$ \\
\hline 3218 & 34.714 & & & & & 0.1 & 0.8 & & & & $T$ \\
\hline 4510 & 34.702 & & & & & -9.6 & 0.8 & & & & $T$ \\
\hline
\end{tabular}

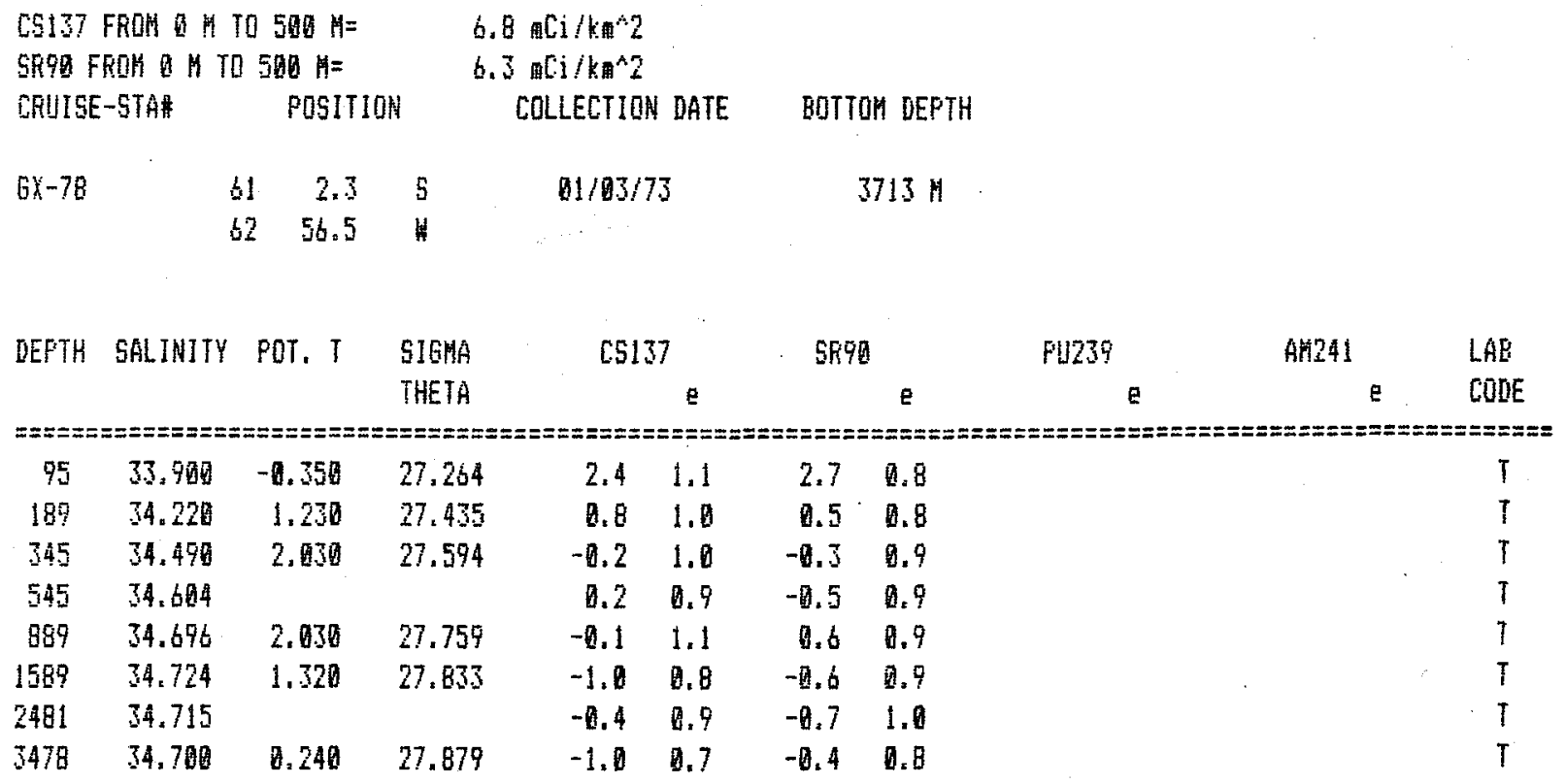

CS137 FROM ON TO $400 \mathrm{M}=$ SR90 FROA D IO $400 \mathrm{H}=$
$2.0 \mathrm{gC \textrm {j }} / \mathrm{km} 2$

$2.0 \mathrm{AC \textrm {i }} / \mathrm{km} 2$ 


\begin{tabular}{|c|c|c|c|c|c|c|c|c|c|c|c|}
\hline \multicolumn{2}{|c|}{ CRUISE-STA } & \multicolumn{2}{|c|}{ POSITION } & \multirow{2}{*}{\multicolumn{2}{|c|}{$\begin{array}{l}\text { COLLECTION DATE } \\
01 / 12 / 73\end{array}$}} & \multirow{2}{*}{\multicolumn{2}{|c|}{$\begin{array}{c}\text { BOTTOM DEPTH } \\
7835\end{array}$}} & & & & \\
\hline GX-82 & & $\begin{array}{ll}56 & 15.0 \\
24 & 59.5\end{array}$ & $\begin{array}{l}5 \\
H\end{array}$ & & & & & & & & \\
\hline DEPTH & SALINITY & POT. T & $\begin{array}{l}\text { SIGMA } \\
\text { THETA }\end{array}$ & \multicolumn{2}{|c|}{$\operatorname{cs} 137$} & \multicolumn{2}{|c|}{ SR99 } & \multicolumn{2}{|c|}{ PU239 } & AM241 & $\angle A B$ \\
\hline \multicolumn{12}{|c|}{ 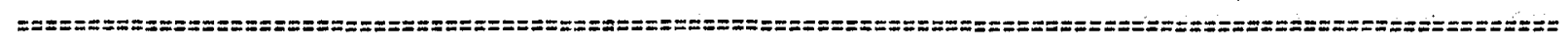 } \\
\hline 12 & 33.727 & 1.320 & 27.834 & 3.0 & 0.3 & 0.9 & 0.1 & 0.840 & 0.950 & & $H$ \\
\hline 52 & 33.737 & 0.910 & 27.067 & & & 1.6 & 1.1 & & & & $T$ \\
\hline 85 & 33.971 & -0.710 & 27.303 & 2.4 & 0.4 & 2.6 & 0.2 & 0.010 & 0.020 & & $H$ \\
\hline 145 & 34.211 & -0.340 & 27.515 & & & 0.8 & 9.8 & & & & $T$ \\
\hline 234 & 34.469 & 0.590 & 27.674 & 1.1 & 0.5 & 1.3 & 9.3 & 0.190 & 0.030 & & $H$ \\
\hline 395 & 34.604 & 0.750 & 27.773 & & & 0.2 & 1.0 & & & & $T$ \\
\hline 500 & 34.698 & 1.530 & 27.798 & 0.6 & 0.4 & 0.1 & 0.3 & & & & $H$ \\
\hline 647 & 34.697 & & & & & 0.3 & 0.8 & & & & $T$ \\
\hline 795 & $34.66 \mathrm{~h}$ & 1.810 & 27.823 & $a .6$ & 0.5 & 0.7 & 0.2 & 0.030 & 0.850 & & H \\
\hline 901 & 34.707 & & & & & -0.2 & 0.7 & & & & $\ddot{T}$ \\
\hline 1585 & 34.665 & & & & & -0.8 & 0.7 & & & & $T$ \\
\hline 2298 & 34.664 & -0.040 & 27.865 & & & -0.5 & 0.8 & & & & $T$ \\
\hline 3664 & 34.652 & -0.510 & 27.878 & & & -0.9 & 0.8 & & & & T \\
\hline 4557 & 34.642 & -0.680 & 27.877 & & & -0.1 & 0.8 & & & & $T$ \\
\hline 6780 & 34.654 & -0.890 & 27.891 & & & -0.2 & 0.9 & & & & $T$ \\
\hline
\end{tabular}

C5137 FFOM OA TO $1000 \mathrm{~K}=$ SR90 FROM O TO $1000 \mathrm{H}=$
4.6 $\mathrm{mCi} / \mathrm{km}^{\wedge} 2$

2.9 町 $/ \mathrm{k}^{\mathrm{N}} 2$ 


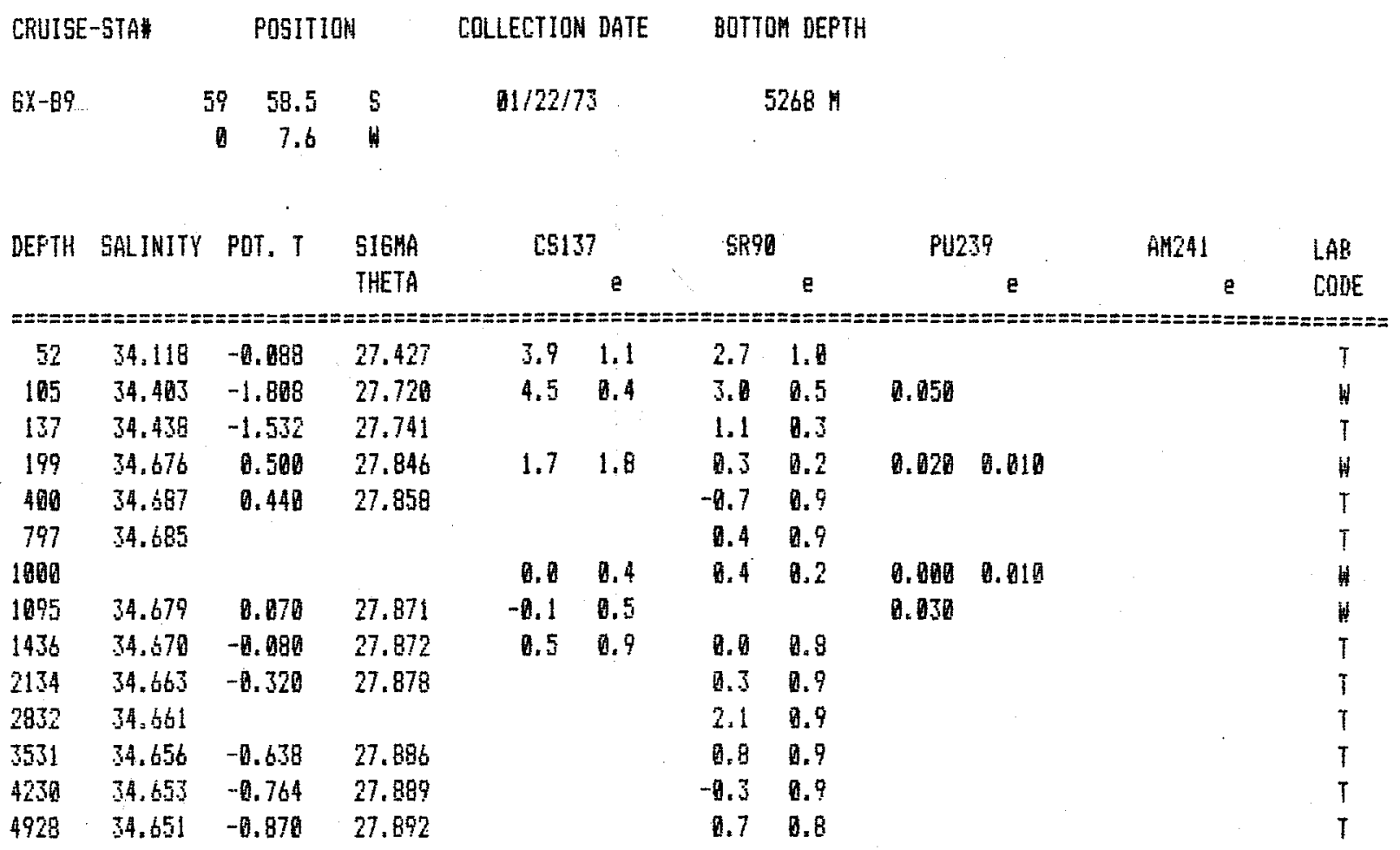

CS137 FROM IO TO $360 \mathrm{M}=$

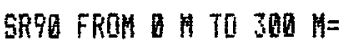

4. $0 . \mathrm{Ci} / \mathrm{km} 2$

$1.9 \mathrm{mLi} / \mathrm{kg}^{\mathrm{A}} 2$ 


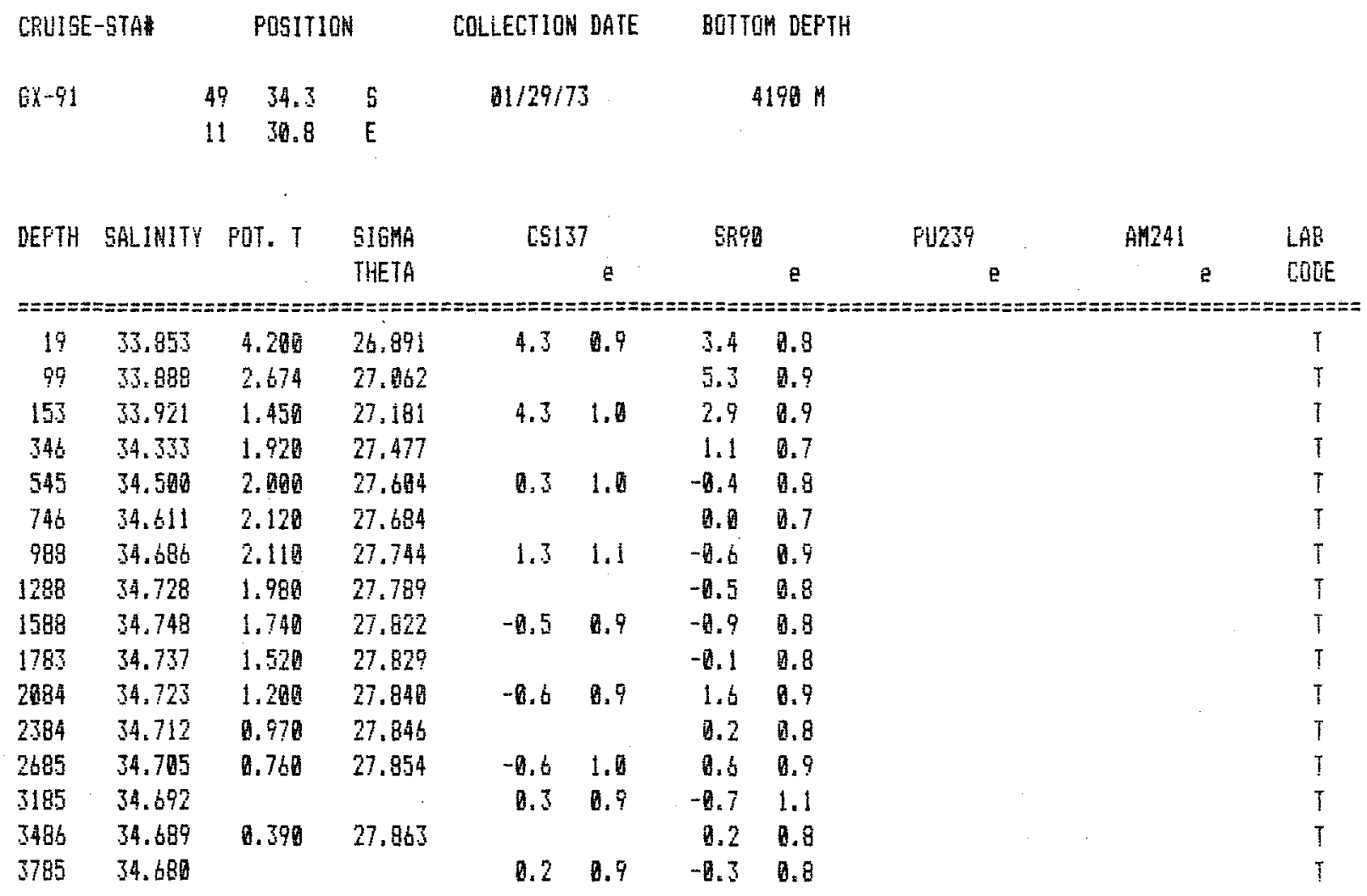

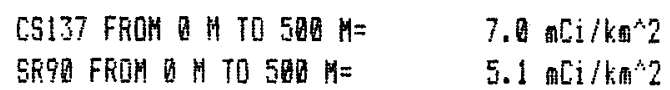

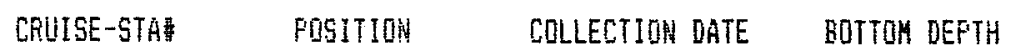

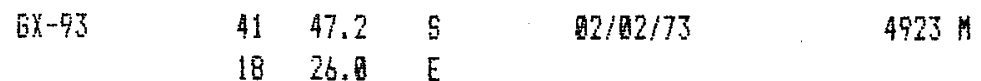

$1826.1 \quad E$

\begin{tabular}{|c|c|c|c|c|c|c|c|c|c|c|c|}
\hline DEFTH & SALINITY & FoT. T & $\begin{array}{l}\text { S1GMA } \\
\text { THETA }\end{array}$ & CS1 & 7 & $5 R 9$ & $g$ & FU: & 39 & AM241 & $\begin{array}{l}\text { LAE } \\
\text { CODE }\end{array}$ \\
\hline \multicolumn{12}{|c|}{ 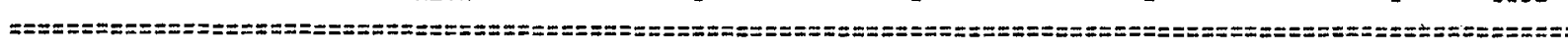 } \\
\hline 昰 & 35.156 & 13.450 & 26.457 & 15.8 & 0.3 & 10.2 & 9.3 & 0.040 & 0.030 & & W \\
\hline 244 & 34.836 & 10.638 & 26.753 & 10.1 & 0.3 & 10.0 & 0.2 & 0.030 & 0.020 & & H \\
\hline 394 & 35.620 & B. 790 & 26.894 & 1.7 & 0.2 & 1.0 & 0,3 & & & & W \\
\hline 740 & 34.375 & & & 2.1 & 0.7 & -7.5 & 0.4 & 0.000 & 0.040 & & W \\
\hline
\end{tabular}

CSI37 FROH O 15 TO 590 SR9O FROH O $M$ TO 500 H=
$20.1 \mathrm{mC \textrm {ikm }} / \mathrm{k}$

$15.3 \mathrm{mL \textrm {j }} / \mathrm{km}^{\mathrm{*} 2}$ 


\begin{tabular}{|c|c|c|c|c|c|c|c|c|c|c|c|}
\hline \multicolumn{2}{|c|}{ CRUISE-STA* } & \multicolumn{2}{|c|}{ POSITION } & \multicolumn{2}{|c|}{ COLLECTION DATE } & \multicolumn{2}{|c|}{ BOTTOM DEPTH } & & & & \\
\hline \multicolumn{2}{|c|}{$6 x-103$} & $23 \quad 59.5$ & 5 & \multicolumn{2}{|c|}{$02 / 17 / 73$} & \multicolumn{2}{|r|}{$4632 \mathrm{M}$} & & & & \\
\hline \multirow[t]{2}{*}{ DEFTH } & \multirow[t]{2}{*}{ SALINHTY } & \multirow[t]{2}{*}{ POT. T } & SIGMA & \multicolumn{2}{|c|}{$[5137$} & \multicolumn{2}{|c|}{ SR9D } & \multicolumn{2}{|c|}{ PU239 } & AM24I & $\angle A B$ \\
\hline & & & THETA & $-1 .-1--$ & e & 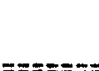 & e & ...-....... & e & e & CODE \\
\hline \multicolumn{12}{|c|}{ 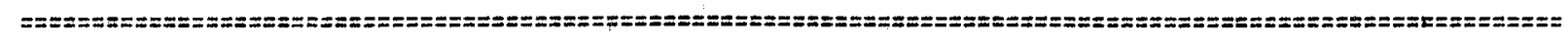 } \\
\hline $\begin{array}{r}3 \\
93\end{array}$ & $\begin{array}{l}35.6104 \\
35.562\end{array}$ & $\begin{array}{l}22.790 \\
16.420\end{array}$ & 26.100 & $\begin{array}{l}16.7 \\
20.8\end{array}$ & $\begin{array}{l}0.4 \\
1.0\end{array}$ & $\begin{array}{l}18.9 \\
14.1\end{array}$ & $\begin{array}{l}0.7 \\
1.1\end{array}$ & & & & th \\
\hline 142 & 35.495 & & & 19.1 & 1.6 & 12.7 & 0.4 & 0.030 & 0.010 & & H \\
\hline 291 & 35.007 & 11.600 & 26.707 & 10.6 & 0.8 & 5.1 & 0.2 & & & & ul \\
\hline 391 & 34.777 & 9.400 & 26.918 & 3.3 & 0.9 & 2.8 & 0.8 & & & & $T$ \\
\hline 489 & 34.606 & 7.480 & 27.081 & 0.6 & 0.3 & 0.8 & 0.2 & 0.050 & 0.010 & & $n$ \\
\hline 690 & 34.417 & & & 0.2 & 0.9 & 0.3 & 0.7 & & & & $T$ \\
\hline 888 & 34.460 & 4.060 & 27.388 & 0.1 & 0.7 & 0.5 & 0.3 & 0.050 & & & $n$ \\
\hline 1088 & 34.542 & & & -9.5 & 0.9 & 0.0 & 8.7 & & & & $T$ \\
\hline 1368 & 34.702 & 3.270 & 27.658 & -8.8 & 1.0 & -0.1 & 0.2 & 0.030 & 0.030 & & $W$ \\
\hline 1665 & 34.936 & & & -1.0 & 0.7 & -8.9 & 0.7 & & & & $T$ \\
\hline 1954 & 34.884 & 2.990 & 27.828 & 2.6 & 1.5 & 8.0 & 0.2 & 0.040 & & & H \\
\hline 2460 & 34.873 & 2.550 & 27.858 & -0.6 & 0.9 & -9.4 & 0.9 & & & & $T$ \\
\hline 2958 & 34.961 & 2.210 & 27.877 & 0.3 & 0.3 & 0.8 & 0.1 & 0.010 & 0.828 & & $W$ \\
\hline 3454 & 34.860 & 2,600 & 27.893 & -0.1 & 1.1 & -0.1 & 0.8 & & & & $T$ \\
\hline 3949 & 34.899 & 1.350 & 27.899 & 0.2 & 0.3 & -9.5 & 0.3 & 0.010 & 0.008 & & $w$ \\
\hline 4248 & 34.752 & 0.830 & 27.887 & -0.4 & 0.8 & 0.1 & 0.8 & & & & $T$ \\
\hline
\end{tabular}

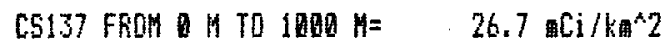

SR90 FROH $\mathrm{H}$ TO $100 \mathrm{H}=17.8 \mathrm{mCi} / \mathrm{km}^{\mathrm{A}} 2$ 


\begin{tabular}{|c|c|c|c|c|c|c|c|c|c|c|}
\hline \multicolumn{2}{|c|}{ CAUISE-STA\# } & \multicolumn{2}{|c|}{ POSITION } & \multicolumn{2}{|c|}{ COLLECTION DATE } & \multicolumn{2}{|c|}{ BOTTOM DEPTH } & & & \\
\hline \multirow[t]{2}{*}{$6 y-107$} & & 0.0 & $s$ & \multicolumn{2}{|c|}{$02 / 22 / 73$} & \multicolumn{2}{|c|}{$5537 \mathrm{H}$} & & & \\
\hline & & 158.5 & $E$ & & & & & & & \\
\hline \multirow[t]{2}{*}{ DEPTH } & \multirow[t]{2}{*}{ SALINITY } & \multirow[t]{2}{*}{ POT. T } & S16月A & \multicolumn{2}{|c|}{$[5137$} & \multicolumn{2}{|c|}{ SROD } & $F \cup 239$ & $\operatorname{Am} 241$ & LAB \\
\hline & & & THETA & & e & & e & e & e & CODE \\
\hline \multicolumn{11}{|c|}{ 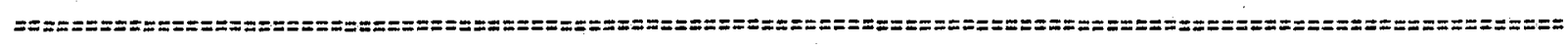 } \\
\hline 48 & 36.565 & 22.150 & 25.384 & 9.9 & 1.1 & 7.6 & 1.? & & & T \\
\hline 147 & 35.269 & 12.350 & 26.718 & 2.8 & 0.9 & 1.7 & 0.8 & & & $T$ \\
\hline 343 & 34.867 & & & 0.2 & 0.9 & -1.0 & 0.8 & & & $T$ \\
\hline 893 & 34.520 & 4.310 & 27.410 & -1.4 & 1.0 & -8.7 & 0.8 & & & $T$ \\
\hline 1350 & 34.848 & 3.690 & 27.734 & -0.1 & 0.9 & -9.5 & 0.8 & & & 1 \\
\hline 2073 & 34.911 & 3.090 & 27.842 & -6.8 & 0.9 & -0.6 & 0.8 & & & 1 \\
\hline 3069 & 34.894 & 2.290 & 27.897 & & & 1.7 & 1,0 & & & $T$ \\
\hline 4061 & 34.884 & 2.000 & 27.911 & & & -0.6 & 8.日 & & & $T$ \\
\hline 5055 & 34.877 & 2.010 & 27.905 & & & -8.5 & 0.7 & & & $T$ \\
\hline
\end{tabular}

CS137 FROM O TO $590 \mathrm{M}=$

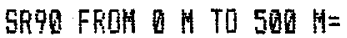

6. $5 \mathrm{mCi} / \mathrm{km} \mathrm{m}^{\mathrm{n}} \mathrm{z}$

4.5 $\mathrm{mC \textrm {j }} / \mathrm{km}^{2} 2$ 


\begin{tabular}{|c|c|c|c|c|c|c|c|c|c|c|c|}
\hline \multicolumn{2}{|c|}{ CFUISE-STA\# } & \multicolumn{2}{|c|}{ POSITION } & \multicolumn{2}{|c|}{ COLLECTION DATE } & \multicolumn{2}{|c|}{ BOTTOM DEPTH } & & & & \\
\hline $6 x-111$ & 1 & $\begin{array}{l}0.5 \\
1.6\end{array}$ & N & \multicolumn{2}{|c|}{$03 / 01 / 73$} & \multicolumn{2}{|c|}{$5153 \mathrm{M}$} & & & & \\
\hline DEPTH & SALINITY & PDT. T & $\begin{array}{l}\text { SIGKA } \\
\text { THETA }\end{array}$ & \multicolumn{2}{|c|}{$\operatorname{cs157}$} & \multicolumn{2}{|c|}{ SR99 } & \multicolumn{2}{|c|}{ PU239 } & AM241 & $\begin{array}{l}\text { LAE } \\
\text { CODEE }\end{array}$ \\
\hline$==$ & $:===$ & $=$ & 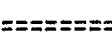 & $:== \pm=$ & $z==z==$ & $=== \pm$ & $y=== \pm$ & :=:=:=z= & :=-z=:= & 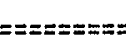 & $==:=2$ \\
\hline 1 & 35.469 & & & 14.7 & 0.2 & 7.9 & 0.2 & 0.030 & 0.009 & & $H$ \\
\hline 50 & 36.024 & & & & & 12.2 & 0.9 & & & & $T$ \\
\hline 190 & 35.532 & & & 2.8 & 0.4 & 3.6 & 0.1 & 0,050 & 0.010 & & $H$ \\
\hline 150 & 35.410 & & & & & 3.3 & 0.9 & & & & $T$ \\
\hline 200 & 35.249 & & & 1.2 & 0.4 & 2.7 & 0.3 & 0.060 & 0.010 & & H \\
\hline 262 & 35.147 & 11.860 & 26.777 & 3.8 & 0.3 & 2.4 & 0.3 & 0.050 & 0.810 & & \# \\
\hline 313 & 35.937 & 10.860 & 26.868 & 2.0 & 0.3 & 0.9 & 0.3 & 0.045 & 0.017 & & H \\
\hline 511 & 34.597 & 6.470 & 27.213 & 0.7 & 0.3 & 0.3 & 0.9 & 0.020 & 0.016 & & 4 \\
\hline 724 & 34.524 & 4.990 & 27.338 & 0.2 & 0.3 & 9.4 & $n .1$ & 9.007 & & & W \\
\hline 931 & 34.594 & 4.320 & 27.467 & 0.7 & 8.3 & $a, \bar{n}$ & 0.3 & 0.030 & 0.010 & & y \\
\hline 1137 & 34.742 & 4.370 & 27.579 & -0.3 & 0.3 & -6.7 & 0.3 & 0.010 & & & $y$ \\
\hline 1445 & 34.958 & 4.060 & 27.783 & 0.3 & 0.3 & 9.0 & 0.2 & 0.006 & 0.0194 & & Wi \\
\hline 1754 & 34.967 & 3.610 & 27.836 & 0.0 & 0.3 & 0.0 & 0.1 & 0.010 & 0.930 & & 4 \\
\hline 2062 & 34.954 & 3.190 & 27.866 & -6.5 & 0.3 & 0.8 & 0.2 & 0.010 & 0.610 & & $W$ \\
\hline 2586 & 34.931 & 2.680 & 27.894 & -0.1 & 0.4 & 0.0 & 0.2 & 0.950 & D. 039 . & & 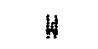 \\
\hline 3100 & 34.915 & 2.386 & 27.906 & 0.2 & 0.3 & 0.6 & 0.2 & 0.010 & 0.010 & & $W$ \\
\hline 3203 & 34.898 & 2.170 & 27.929 & 0.0 & 0.3 & $-1,4$ & 0.2 & 0.053 & 0.013 & & $W$ \\
\hline 4130 & 34.897 & 1.960 & 27.917 & 0.2 & 0.3 & -6.1 & 0.2 & 0.010 & 0.010 & & 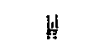 \\
\hline 4336 & 34.890 & 1.860 & 27.919 & -8.3 & 0.3 & -4.3 & 0.3 & 9.003 & 0.005 & & 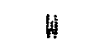 \\
\hline 4541 & 34.973 & 1.810 & 27.917 & -0.1 & 0.6 & 9.4 & 0.6 & 0.010 & 0.020 & & h \\
\hline 4748 & 34.868 & 1.790 & 27.915 & 0.0 & 0.3 & 0.2 & 0.3 & 0.007 & 0.011 & & $\psi$ \\
\hline 4952 & 34.884 & 1.730 & 27.916 & 0.4 & 0.3 & 1.3 & 0.3 & 0.020 & 0.010 & & $W$ \\
\hline 5158 & 34.961 & 1.700 & 27.916 & -0.3 & 0.3 & 2.5 & 0.2 & 0.005 & 0.008 & & $y$ \\
\hline
\end{tabular}

[5137 FROM O TO $750 M=$ SRPQ FrOH \& $7075 \mathrm{H}$ 月 FULST FROM O 10 TO $5163 \mathrm{M}=$
7.9 解 $\mathrm{i} / \mathrm{km} 2$

$7.5 \mathrm{mi \textrm {i }} / \mathrm{km}^{2} 2$

4. $42 \mathrm{mCl} / \mathrm{kn}^{\circ} 2$ 


\begin{tabular}{|c|c|c|c|c|c|c|c|c|c|c|c|}
\hline \multicolumn{2}{|c|}{ CFUISE-STA\# } & \multicolumn{2}{|c|}{ PasITIOn } & \multicolumn{2}{|c|}{ COLLECTION DATE } & \multicolumn{2}{|c|}{ BOTTOM DEFTH } & & & & \\
\hline \multirow[t]{2}{*}{$6 x-113$} & & 10 59.1 & 盟 & \multicolumn{2}{|c|}{$03 / 05 / 73$} & \multicolumn{2}{|c|}{4948} & & & & \\
\hline & & 2031.0 & H & & & & & & & & \\
\hline \multirow[t]{2}{*}{ DEFTH } & \multirow[t]{2}{*}{ SALINITY } & \multirow[t]{2}{*}{ POT. T } & SIGHA & \multicolumn{2}{|c|}{$[5137$} & \multirow[t]{2}{*}{ SF 9} & & \multicolumn{2}{|c|}{ FU239 } & A 1241 & LAE \\
\hline & & & THETA & & e & & e & & $\mathrm{e}$ & e & CONE \\
\hline \multicolumn{12}{|c|}{ 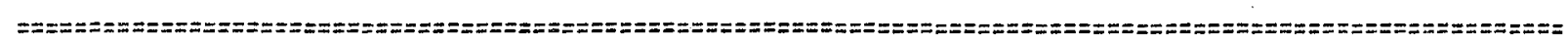 } \\
\hline 1 & 35.774 & & & 4.5 & 0.6 & 4.3 & 0.2 & 0.19日0 & 0.620 & & H \\
\hline 150 & 35.428 & & & 5.6 & 0.9 & 2.3 & 0.8 & & & & $T$ \\
\hline 310 & 35.129 & 10.470 & 27.689 & & & -0.3 & 1.1 & & & & $T$ \\
\hline 413 & 35.054 & 9.540 & 27.111 & 1.0 & 0.2 & 1.0 & & 9.920 & 0.010 & & $H$ \\
\hline 516 & 35.617 & 8.730 & 27.214 & 1.6 & 0.8 & 1.1 & Q. & & & & $T$ \\
\hline 928 & 34.759 & 5.480 & 27.466 & & & -9.5 & 0.9 & & & & $T$ \\
\hline 1442 & 34.947 & 4.170 & 27.779 & 2.0 & 0.9 & 1.5 & 0.9 & & & & $T$ \\
\hline 2198 & 34.955 & 3.010 & 27.879 & & & 0.8 & 0.8 & & & & $T$ \\
\hline 3158 & 34.919 & & & & & -0.4 & 1.0 & & & & $T$ \\
\hline 4145 & 37.893 & 1.920 & 27.925 & & & -0.3 & 0.9 & & & & $T$ \\
\hline 4542 & 34.883 & 1.900 & 27.719 & & & -8.4 & 0.8 & & & & $\mathrm{~T}$ \\
\hline
\end{tabular}

CS137 FROM O I TO $300 \mathrm{H}=$ SR9G FROH O 10 TO $300 \%=$
$6.9 \curvearrowleft \mathrm{Ci} / \mathrm{kn}$

3.1 盛 $/ \mathrm{kf}_{\mathrm{f}} \div 2$ 


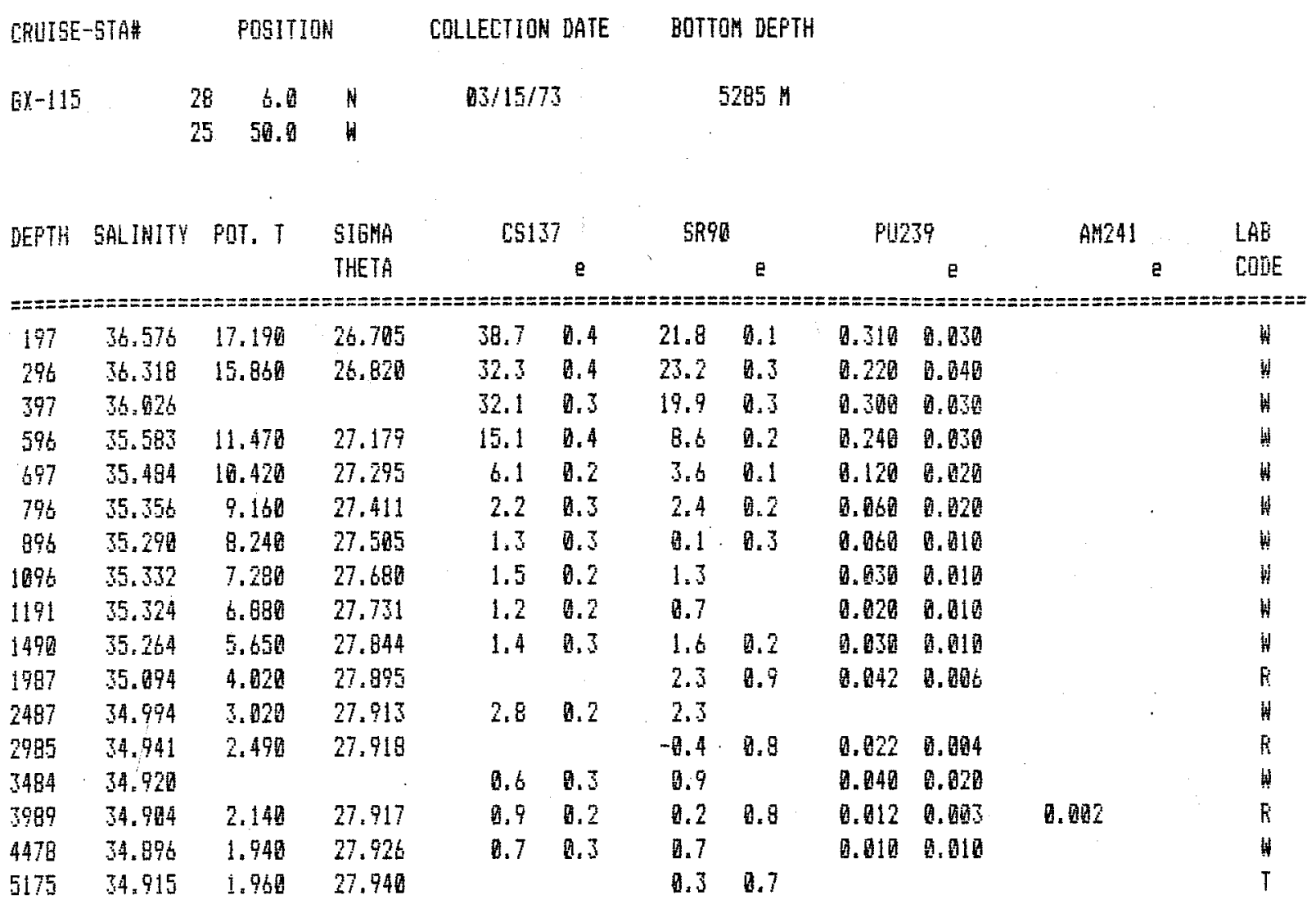

CS137 Froh o $\mathrm{AlO}$ 3000 $\mathrm{H}=\quad 112.8$ aCi $/ \mathrm{km}^{\mathrm{A}} 2$

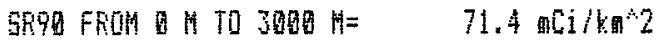

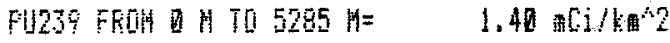




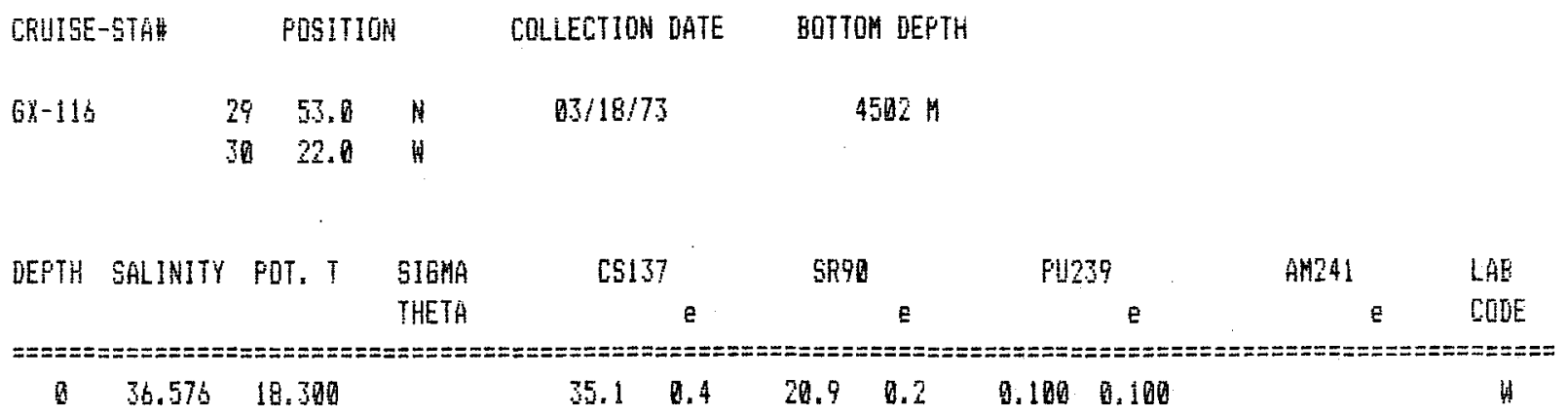

CRUISE-STA\# POSITION COLLETION DATE EDTTOH DEFTH

\begin{tabular}{|c|c|c|c|}
\hline $6 x-117$ & 30 & 44.5 & $H$ \\
\hline
\end{tabular}

\begin{tabular}{|c|c|c|c|c|c|c|c|c|c|c|c|}
\hline DEFTH & CALIHITY & POT. T & $\begin{array}{l}\text { SIGHA } \\
\text { THETA }\end{array}$ & \multicolumn{2}{|c|}{$\operatorname{cs157}$} & \multicolumn{2}{|c|}{5890} & \multicolumn{2}{|c|}{ PU239 } & $\mathrm{AH}_{24} \mathrm{~L}$ & $\begin{array}{l}\angle A E \\
\text { CONE }\end{array}$ \\
\hline$z==z==$ & $===ニ= \pm==z=$ & $:=z=z=-z$ & $==\pi= \pm==$ & $== \pm==$ & $== \pm=z$ & $z== \pm= \pm$ & $z=== \pm$ & $z==-=$ & $=== \pm==8$ & $== \pm=:== \pm=$ = & 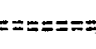 \\
\hline 2 & 36.739 & 19.5010 & 26.215 & 35.5 & 0.4 & 22.2 & 0.3 & & & & $W$ \\
\hline 100 & 36.733 & 19.366 & 26.272 & & & 20.5 & 1.1 & & & & 1 \\
\hline 295 & 36.493 & 17,016 & 26.613 & 34,0 & 0.6 & 19.5 & 0.2 & 0.310 & 0.036 & & in \\
\hline 393 & 36.176 & 15.680 & 26.754 & & & 20.5 & 0.9 & & & & $T$ \\
\hline 5.59 & 35.983 & 13.800 & 23.944 & 26.6 & 0.3 & 15.8 & 0.2 & 0.258 & 0.840 & & $y$ \\
\hline 686 & 35.607 & 11.770 & 27.142 & & & 10.2 & 1.4 & & & & $T$ \\
\hline 1076 & 35.525 & 5.981 & 27.875 & 3.1 & 0.3 & 2.1 & 0.1 & & & & $\omega$ \\
\hline 1215 & 35.231 & 6.200 & 27.749 & & & 1.8 & 0.9 & & & & $T$ \\
\hline 1474 & 35.197 & 5.220 & 27.844 & & & 1.7 & 9.8 & & & & $\bar{i}$ \\
\hline 1954 & 35.095 & 3.940 & 27.905 & 3.3 & 0.9 & 0.7 & 0.4 & 0.950 & 0.010 & & 19. \\
\hline 2506 & 34.972 & 2.970 & 27.900 & & & 1.0 & 0.8 & & & & T \\
\hline 3425 & 34.926 & 2,410 & 27.912 & & & $-1,4$ & 1.3 & & & & $T$ \\
\hline
\end{tabular}


$-47-$

SECTION 2

Radiochemical Data (GEOSECS Pacific) 


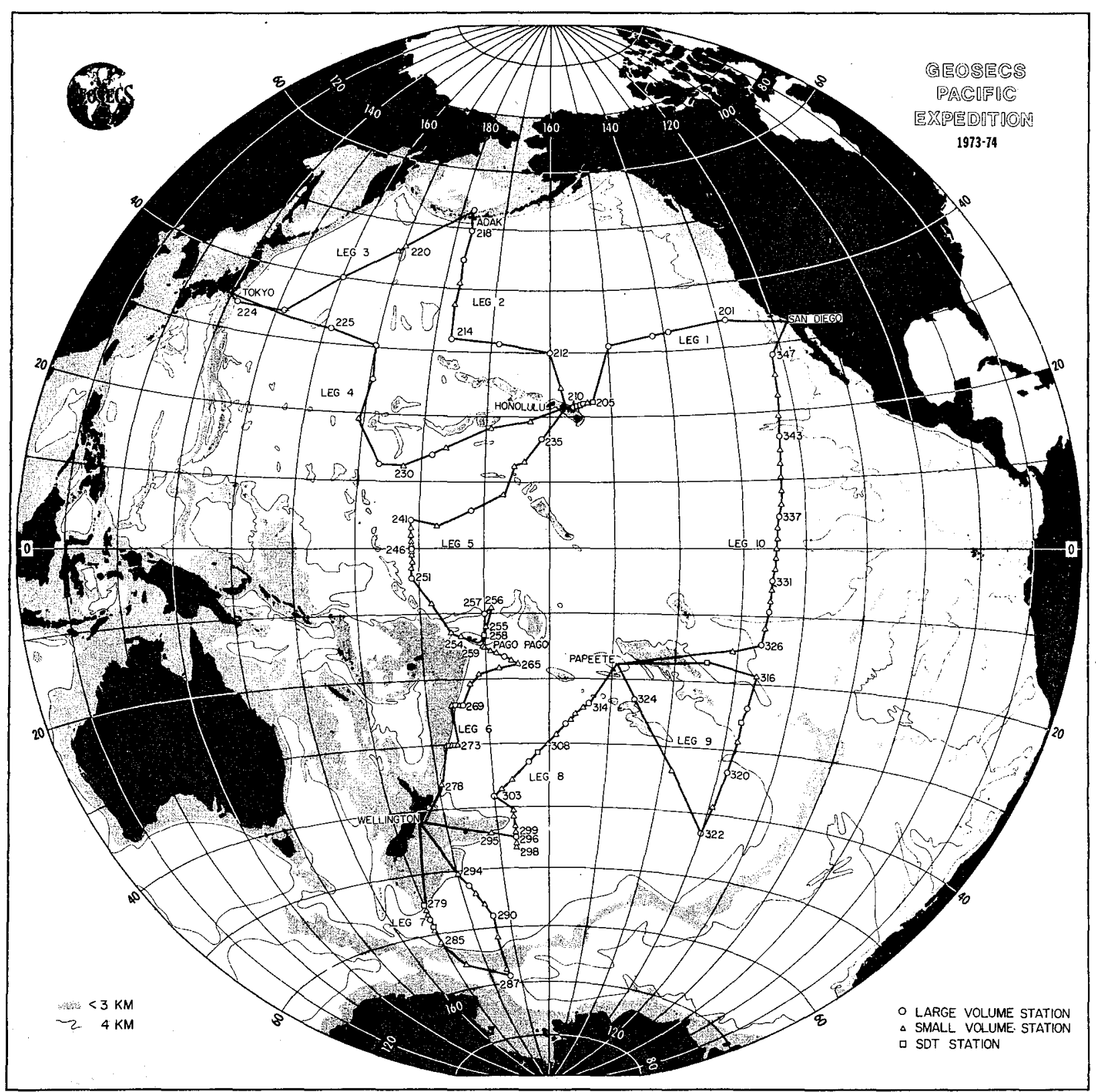




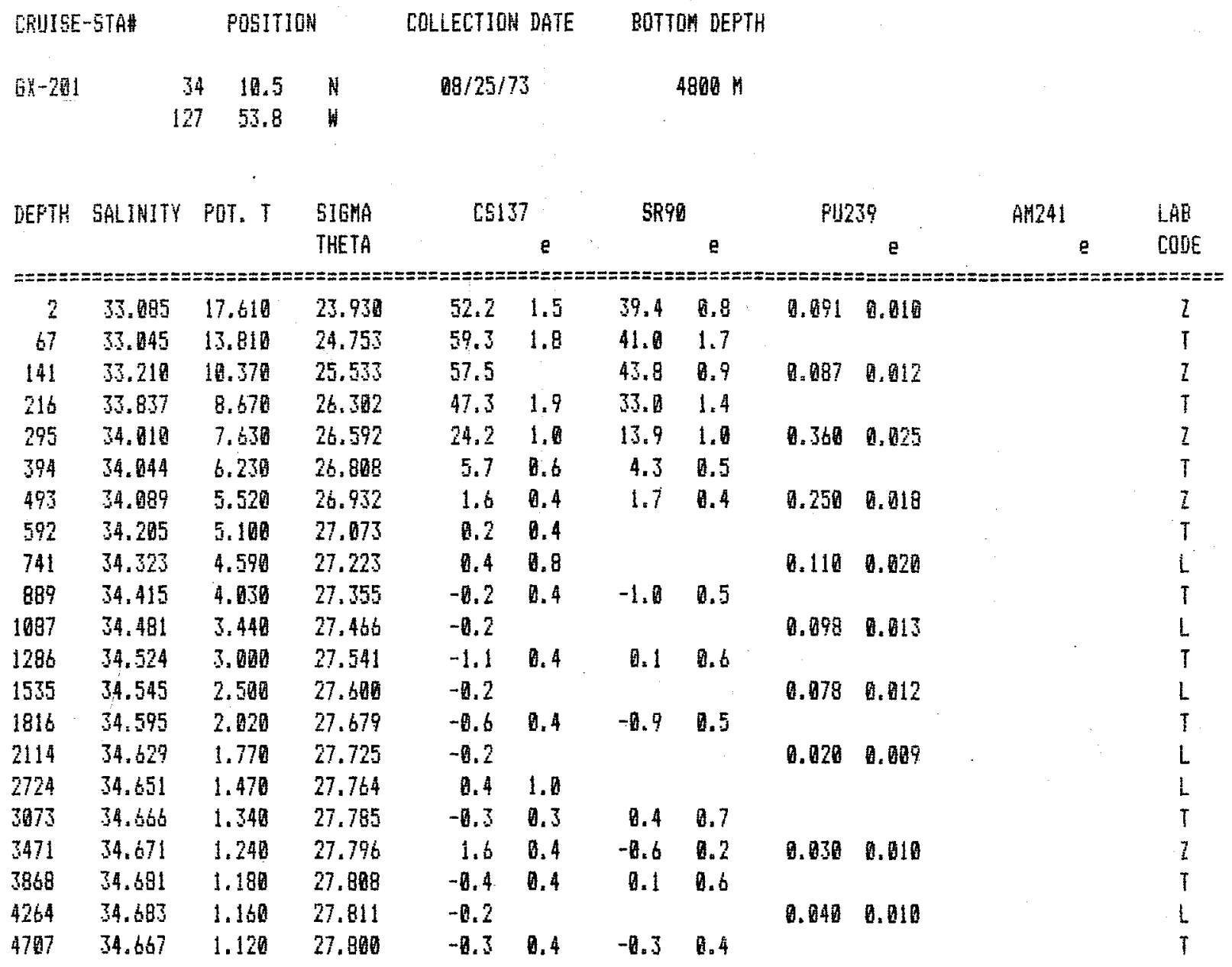

CS137 FROM O HO TO 1 OOOH $M=$ SR90 FEOK O TO $1000 \mathrm{M}=$ PU259 FROM I IO $4890 \mathrm{M}=$
$75.7 \mathrm{mCl} / \mathrm{k}^{\wedge} \mathrm{A} 2$

54.5 mCi $/ \mathrm{Km}^{\wedge}$ ?

$1.53 \mathrm{mC \textrm {H } / \mathrm { km } / 2}$ 


\begin{tabular}{|c|c|c|c|c|c|c|c|c|c|c|c|}
\hline \multicolumn{2}{|c|}{ CRUISE-STA\# } & \multicolumn{2}{|c|}{ POSITION } & \multicolumn{2}{|c|}{ COLLECTION DATE } & \multicolumn{2}{|c|}{ BOTTOM DEPTH } & & & & \\
\hline EX-20:2 & $\begin{array}{r}33 \\
139\end{array}$ & $\begin{array}{rr}3 & 6.0 \\
9 & 34.4\end{array}$ & $N$ & \multicolumn{2}{|c|}{$08 / 30 / 73$} & \multicolumn{2}{|c|}{$5129 \mathrm{M}$} & & & & \\
\hline DEPTH & SALINITY & PQT. T & $\begin{array}{l}\text { SIGMA } \\
\text { THETA }\end{array}$ & \multicolumn{2}{|c|}{$[5137$} & \multicolumn{2}{|c|}{$5 R 90$} & \multicolumn{2}{|c|}{ P11239 } & AM241 & $\begin{array}{l}\text { LAE } \\
\text { CODE }\end{array}$ \\
\hline \multicolumn{11}{|c|}{ 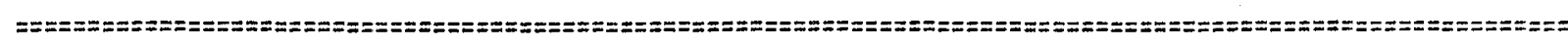 } & \\
\hline 15 & 34.451 & 21.630 & 24.079 & 54.7 & 7.0 & 44.8 & 0.7 & 0.040 & 0.020 & & H \\
\hline 70 & 34.289 & 16.550 & 25,104 & 58.1 & 1.7 & 41.6 & 9.9 & & & & $T$ \\
\hline 141 & 34.439 & 14.920 & 25.596 & 59.7 & 0.7 & 44.0 & 0.4 & 0.079 & 0.020 & & H \\
\hline 240 & 34.151 & 10.530 & 26,238 & 51.6 & 1.0 & 39.4 & 2.0 & & & & $T$ \\
\hline 346 & 34.748 & 8.730 & 26.456 & 37.8 & 1.0 & 29.8 & 0.9 & 0.450 & 0.930 & & 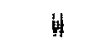 \\
\hline 496 & 33.969 & 6.070 & 26.711 & & & 6.3 & 0.6 & & & & $T$ \\
\hline 645 & 34.966 & 4.567 & 27.023 & & & 1.1 & 0.2 & 0.390 & 0.230 & & 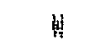 \\
\hline 844 & 34.288 & 4.959 & 27.252 & 2.4 & 0.4 & 0.5 & 0.3 & & & & $T$ \\
\hline 1045 & 34.415 & 3.630 & 27.395 & -6.8 & 0.9 & -0.5 & 0.2 & 0.080 & 0.630 & & $\omega$ \\
\hline 1246 & 34.487 & 3.120 & 27.590 & & & 0.0 & 0.2 & & & & $T$ \\
\hline 1446 & 34.526 & 2.710 & 27.568 & -1.4 & 1.0 & -0.3 & 0.5 & 0.060 & 0.047 & & $H$ \\
\hline 1646 & 34.560 & 2.340 & 27.625 & -0.2 & 0.4 & -0.3 & 0.3 & & & & $T$ \\
\hline 1847 & 34.584 & 2, 000 & 27.671 & 0.0 & 0.5 & -9.4 & 0.5 & 0.880 & 0.016 & & 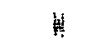 \\
\hline $213 b$ & 34.624 & 1.710 & 27.725 & & & -0.1 & 0.8 & & & & 1 \\
\hline 2437 & 34.644 & 1.560 & 27.752 & 1.0 & 0.8 & -10.7 & 0.5 & 0.050 & 0.025 & & \# \\
\hline 2736 & 34.570 & 1.410 & 27.763 & 1.0 & 0.4 & 0.3 & 0.2 & & & & $T$ \\
\hline 3060 & 34.671 & 1.340 & 27.797 & -0.3 & 0.9 & -6.4 & 0.5 & 0.050 & 0.010 & & 4 \\
\hline 30EA & 34.654 & 1.320 & 27.777 & -0.4 & 0.7 & 0.4 & 0.4 & & & & $T$ \\
\hline 3431 & 34.673 & 1.210 & 27.799 & -9.7 & 0.6 & -9.1 & 0.1 & 0.010 & 0.010 & & is \\
\hline 377 & 34.673 & 1.180 & 27.692 & -0.5 & 0.4 & -0.4 & 0.3 & & & & $T$ \\
\hline 4122 & 34.685 & 1.160 & 27.813 & -0.8 & 0.9 & -9.8 & 0.5 & 0.040 & 0.020 & & in \\
\hline 4524 & 34.681 & 1.120 & 27.812 & -0.3 & 0.3 & -0.8 & 0.6 & & & & $T$ \\
\hline
\end{tabular}

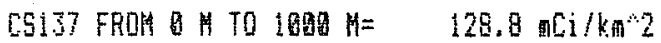
5F90 FFOM ID $1000 \mathrm{M}=78.2 \mathrm{aCi} / \mathrm{km}^{\wedge} 2$

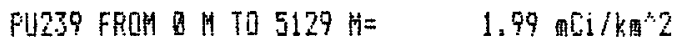




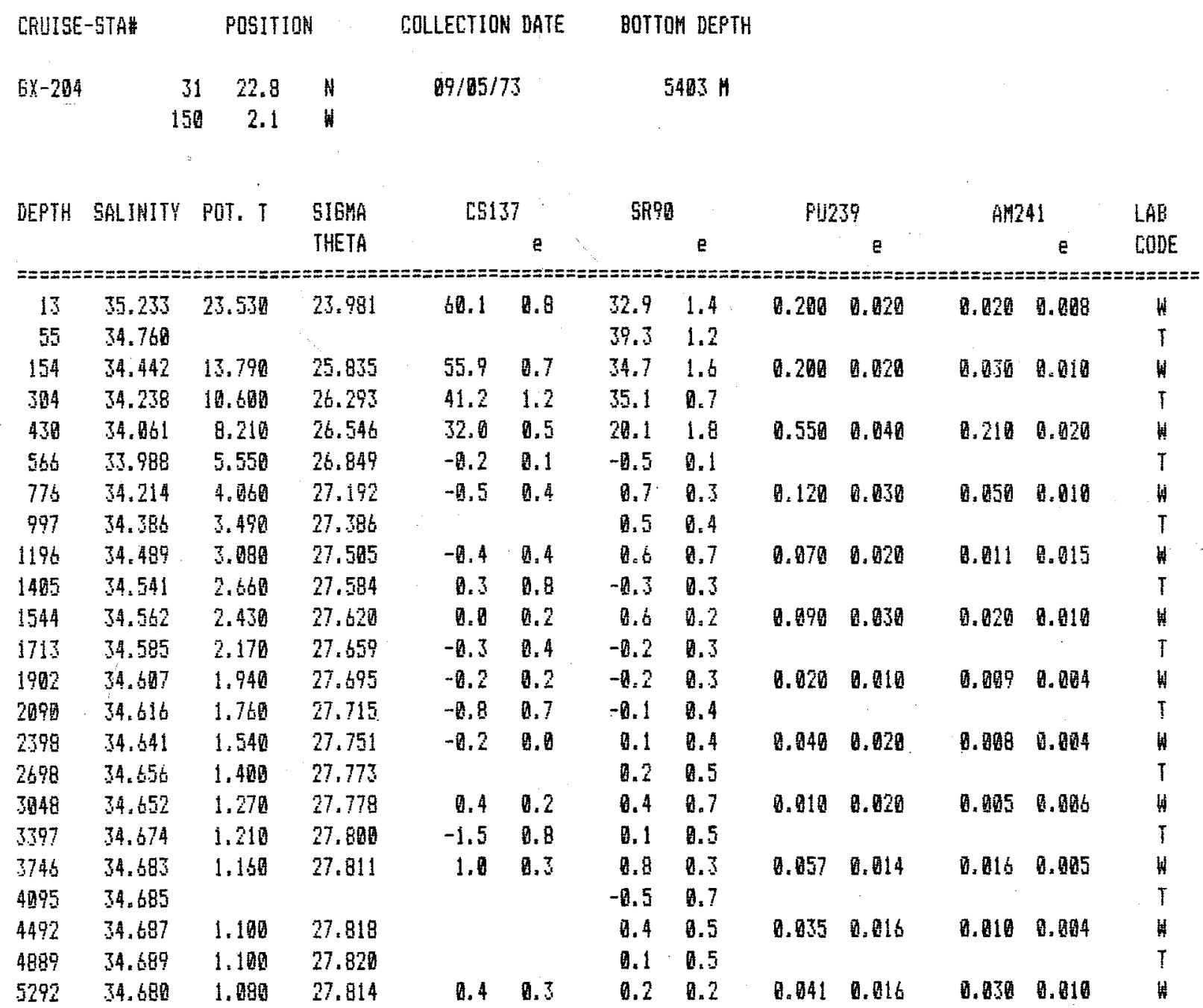

[5137 FHOM O 10 600 $\mathrm{H}=$

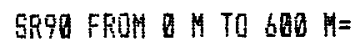
PU239 FROM O 4 TO 5403 H= A月241 FROM II $M$ TO $5405 \mathrm{H}=$

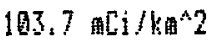

70.7 敗 $\mathrm{i} / \mathrm{km} 2$

2.08 再 $\mathrm{Ci} / \mathrm{km} \mathrm{B}^{2} 2$

$0.68 \mathrm{mCi} / \mathrm{kn}^{\mathrm{N}} 2$ 


\begin{tabular}{|c|c|c|c|c|c|c|c|c|c|c|c|c|}
\hline \multicolumn{2}{|c|}{ CRUISE-STA } & \multicolumn{2}{|c|}{ PQSITION } & \multicolumn{2}{|c|}{ COLLECTION DATE } & \multicolumn{2}{|c|}{ BOTTOH DEFTH } & & & & & \\
\hline $6 x-212$ & $\begin{array}{r}3 \\
15\end{array}$ & $\begin{array}{rr}0.0 \\
69 & 50.5\end{array}$ & H & \multicolumn{2}{|c|}{$89 / 18 / 73$} & \multicolumn{2}{|c|}{$5877 \mathrm{M}$} & & & & & \\
\hline DEFTH & SALIHITY & POT. T & $\begin{array}{l}\text { SIGMA } \\
\text { THETA }\end{array}$ & \multicolumn{2}{|c|}{58137} & \multicolumn{2}{|c|}{$5 R 90$} & \multicolumn{2}{|c|}{ PU239 } & \multicolumn{2}{|c|}{ AM?41 } & $\begin{array}{l}\text { LAE } \\
\text { CODE }\end{array}$ \\
\hline$==x$ & $\Rightarrow= \pm=$ & $z==$ & $=z==:=z=$ & & -0 & 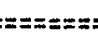 & $== \pm$ & - & $== \pm= \pm$ & $:= \pm=0$ & $=:=z= \pm=$ & $=z= \pm=$ \\
\hline 10 & 35.470 & 25.200 & 23.666 & 59.8 & 0.5 & 35.1 & 0.9 & 0.170 & 0.020 & 0.990 & 0.100 & प \\
\hline 60 & 34.990 & 17.700 & 25.369 & 55.5 & & & & 0.200 & 0.070 & & & $L$ \\
\hline 120 & 34.670 & 15,000 & 25.752 & 53.5 & 0.5 & 29.0 & 0.3 & 0.110 & 0.020 & 0.030 & 0.020 & $H$ \\
\hline 190 & 34.390 & 12.900 & 25.972 & 54.9 & 1.0 & & & 0.210 & 0.08 & & & L \\
\hline 260 & 34.270 & 11.300 & 26.201 & 58.9 & 0.5 & 31.3 & 0.4 & 0.240 & 0.030 & 0.090 & 0.820 & H \\
\hline 426 & 34.128 & 9.078 & 26.465 & 30.5 & 0.3 & & & 0.730 & 0.060 & & & $H$ \\
\hline 595 & 34,206 & 6.610 & 26.806 & 6.1 & 0.3 & 5.9 & 0.4 & 0.510 & 0.940 & 0.120 & 0.020 & 其 \\
\hline 956 & 34.321 & 3.650 & 27.319 & -8.1 & 0.3 & 0.2 & 0.5 & 0.100 & 0.620 & 0.019 & 9.910 & $H$ \\
\hline 1391 & 34.520 & 2.740 & 27.558 & 0.1 & 0.2 & 0.4 & 0.4 & 0.059 & 0.040 & 0.840 & 0.030 & H \\
\hline 1790 & 34.585 & 2.140 & 27.661 & -0.3 & 0.3 & -0.1 & 0.4 & 0.1840 & 0.040 & 0.015 & 0.005 & $H$ \\
\hline 2442 & 34.539 & 1.540 & 27.742 & 0.9 & 0.3 & 8. 6 & 0.5 & 0.850 & 0.020 & 0.020 & & $H$ \\
\hline 3241 & 34.655 & 1.270 & 27.781 & 8.8 & 0.3 & 0.8 & 0.3 & & & 0.020 & 0.010 & 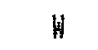 \\
\hline 4039 & 34.656 & 1.160 & 27.789 & 1.0 & 0.3 & 1.2 & 0.2 & 0.980 & 0. 1040 & 0.010 & 0.010 & W \\
\hline 4836 & 34.686 & 1.890 & 27.818 & -0.2 & 0.2 & 0.1 & 0.4 & 0.050 & & 0.010 & 1. D19 & 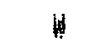 \\
\hline 5238 & 34.697 & 1.800 & 27.819 & -0.3 & 0.2 & & & 0.086 & 0.014 & & & W \\
\hline 5638 & 34.674 & 1.080 & 27.889 & 0.4 & 0.2 & 0.0 & 0.7 & 0.140 & 0.030 & 0.930 & 0. 1010 & 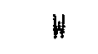 \\
\hline
\end{tabular}

C5137 FROM O 1 To $1000 \mathrm{M}=$ SROG FFOH I I0 $1000 \mathrm{M}=$ PU23 FROM O 105877 H= AM241 FROM O 14 TO 5877 前=
$117.8 \mathrm{MCi} / \mathrm{km}$. 2 $69.5 \mathrm{mCl} / \mathrm{kt} / 2$

3.68 听 $/ \mathrm{km}^{*} 2$

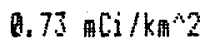




\begin{tabular}{|c|c|c|c|c|c|c|c|c|c|c|c|c|}
\hline \multicolumn{2}{|c|}{ CRUISE-GTA\# } & \multicolumn{2}{|c|}{ POSITIOE } & \multicolumn{2}{|c|}{ CDLLECTION DATE } & \multicolumn{2}{|c|}{ BOTTOM DEFTH } & & & & & \\
\hline \multirow[t]{2}{*}{ Gi -213} & \multirow{2}{*}{$\begin{array}{r}36 \\
168\end{array}$} & 58.1 & H & \multirow{2}{*}{\multicolumn{2}{|c|}{$09 / 22 / 73$}} & \multicolumn{2}{|c|}{$5761 M$} & & & & & \\
\hline & & 8828.5 & H & & & & & & & & \\
\hline \multirow[t]{2}{*}{ DEFTH } & \multirow[t]{2}{*}{ SALIHITY } & \multirow[t]{2}{*}{ POT. T } & SIEKA & \multirow{2}{*}{\multicolumn{2}{|c|}{ C5137 }} & \multirow[t]{2}{*}{$5 R 90$} & & \multicolumn{2}{|c|}{ PIJ 239} & \multicolumn{2}{|c|}{ AM24! } & \multirow{2}{*}{$\begin{array}{l}\text { LAB } \\
\text { CODE }\end{array}$} \\
\hline & & & THETA & & & & E & & e & & e & \\
\hline \multicolumn{13}{|c|}{ 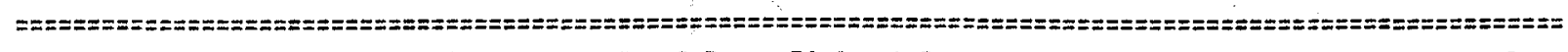 } \\
\hline 20 & 35.510 & 26.800 & 23.285 & 50.3 & 0.5 & 34.4 & 1.8 & & & & & $T$ \\
\hline 60 & 34.870 & 21.100 & 24.390 & 50.3 & 0.4 & 34.6 & 0.5 & 0.080 & 0.010 & & & $H$ \\
\hline 95 & 34.779 & 17.620 & 25.215 & 48.3 & 0.4 & 29.1 & 1.3 & 0.070 & 0.010 & & & H \\
\hline 176 & 34.604 & 14.660 & 25.773 & 53.5 & 0.5 & 36.6 & 0.8 & & & & & $T$ \\
\hline 257 & 34.457 & 13.060 & 25.996 & 48.2 & 0.5 & 29.9 & 0.2 & 0.380 & 0.030 & 0.040 & 0.010 & 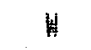 \\
\hline 337 & 34,363 & 11.840 & 26.163 & 48.2 & 1.0 & & & & & & & $T$ \\
\hline 448 & 34.170 & 9.446 & $2 b .435$ & 36.1 & 0.4 & 22.7 & 0.6 & 0.820 & 8. 1900 & 0.3700 & 0.200 & 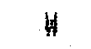 \\
\hline 626 & 34.133 & 6.270 & 26.796 & 11.9 & 0.7 & 7.9 & 1.0 & & & & & $T$ \\
\hline 831 & 34.153 & 4.340 & 27.115 & -0.5 & 0.4 & -0.5 & 0.2 & 9.230 & 0.020 & 0.050 & 0.050 & $H$ \\
\hline 1044 & 34.336 & & & & & -0.4 & 0.3 & & & & & $T$ \\
\hline 1197 & $34.42 b$ & 3.180 & 27.446 & 0.2 & 0.3 & 0.5 & 0.3 & 0.090 & 0.020 & & & 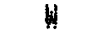 \\
\hline 1391 & 34.499 & 2.820 & 27.536 & & & 10.6 & 0.3 & & & & & 1 \\
\hline 1590 & 34.544 & 2,460 & 27.603 & 8.9 & 9.4 & 0.0 & 0.3 & 0.870 & 0.010 & 0.030 & 9.050 & H \\
\hline 1787 & 34.573 & 2,140 & 27.652 & & & -8.4 & 0.5 & & & & & $T$ \\
\hline 2136 & 34.617 & $1.72 \theta$ & 27.719 & -8.6 & 0.4 & -0.2 & 0.4 & & & & & $n$ \\
\hline 2434 & 34.634 & 1.580 & 27.742 & & & -0.2 & 0.4 & & & & & $T$ \\
\hline 2823 & 34.651 & 1.410 & 27.768 & 0.2 & 0.3 & -0.2 & 0.1 & & & & & 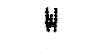 \\
\hline 3522 & 34.674 & 1.220 & 27.860 & -9.2 & 0.2 & 0.3 & 0.2 & & & & & $W$ \\
\hline 4418 & 34.683 & 1.130 & 27.813 & -9.4 & 0.2 & 0.1 & 0.4 & & & & & H \\
\hline 4817 & 34.686 & 1.100 & 27.817 & & & -0.5 & 8.4 & & & & & $T$ \\
\hline 5216 & 34.683 & 1.090 & 27.916 & -10.1 & 0.2 & -0.4 & 0.2 & 9.129 & 0.840 & 0.050 & 0.010 & $H$ \\
\hline 5614 & 34.683 & 1.040 & 27.818 & & & -1.1 & 8.1 & & & & & $T$ \\
\hline
\end{tabular}

CS137 FFOM OH TO $5761 \mathrm{M}=124.6 \mathrm{ACi} / \mathrm{km} 2$

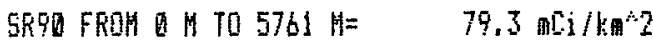




$\begin{array}{lrrrcc}\text { CRUISE-GTAR } & & \text { PISITION } & \text { COLLECTIDN DATE } & \text { BOTTOM DEFTH } \\ \text { GX-214 } & 32 & 1.5 & N & 09 / 25 / 73 & 5706 \mathrm{M} \\ & 176 & 59.9 & & & \end{array}$

\begin{tabular}{|c|c|c|c|c|c|c|c|c|c|c|c|c|}
\hline DEPTH & SALINITY & POT. T & $\begin{array}{l}\text { SIGMA } \\
\text { THETA }\end{array}$ & \multicolumn{2}{|c|}{05137} & SRq & e & PU239 & 39 & \multicolumn{2}{|c|}{ AM241 } & $\begin{array}{l}\text { LAB } \\
\text { CODE }\end{array}$ \\
\hline :=E & $z==$ & & $z==z==$ & $:===$ & $====$ & $=== \pm=$ & $z== \pm$ & $z== \pm=x$ & $=z= \pm==z$ & $======8$ & $=z=z===$ & $=z=z==$ \\
\hline 29 & 34.569 & 25.800 & 22.799 & 58.8 & 1.0 & 34,4 & 1.5 & 0.109 & 0.013 & & & $k$ \\
\hline 60 & 34,700 & 17.700 & 25,144 & 53.2 & 1.1 & 35.6 & 1.4 & & & & & $T$ \\
\hline 105 & 34.676 & 15.8Q0 & 25.573 & 54.2 & 0.4 & 29.1 & 0.8 & 0.249 & 0.060 & 0.019 & 0.908 & $\psi$ \\
\hline 155 & 34.571 & 14.460 & 25.791 & & & 32.8 & 2.0 & & & & & $T$ \\
\hline 216 & 34.463 & 13.190 & 25.975 & 41.3 & 0.4 & 29.6 & 9.5 & 0.275 & 0.021 & & & y \\
\hline 276 & 34.403 & 12,310 & 26.183 & 44,9 & 0.4 & 31.0 & 0.4 & 0.390 & 0.070 & 0.026 & 0.089 & 4 \\
\hline 345 & 34.324 & 11.298 & 26.236 & 38.6 & 0.4 & $2 b .5$ & 1.2 & 0.400 & 0.050 & & & V \\
\hline 474 & 34.174 & 9.046 & 26.506 & 32.1 & 0.4 & 21.0 & 0.2 & 0.590 & 0.100 & 0.062 & 0.012 & 男 \\
\hline 628 & 34.034 & 6.330 & 26.789 & 12.9 & 0.3 & 10.6 & 0.3 & 0.520 & 0.040 & & & $W$ \\
\hline 777 & 34.119 & 4.620 & 27.052 & 2.4 & 0.5 & 2.5 & 0.2 & 0.210 & 0.050 & 0.120 & 0.020 & 6 \\
\hline 896 & 34.222 & 3.940 & 27.211 & 4.7 & 0.5 & 2.1 & 0.5 & & & & & $T$ \\
\hline 1191 & 34.412 & 3.040 & 27.448 & D.8 & 0.3 & 0.9 & 0.2 & 0.160 & 0.030 & 0.043 & 0.909 & $H$ \\
\hline 1840 & 34.569 & 1.990 & 27.641 & 0.2 & 0.4 & -0.1 & 0.2 & 0.694 & 0.022 & Q. .3 .31 & 9.608 & W \\
\hline 2542 & 34.631 & 1.480 & 27.747 & 0.8 & 0.3 & 0.3 & 0.2 & 0.980 & 0.010 & & & W \\
\hline 3341 & 34.663 & 1.220 & 27.791 & & & & & 0.060 & 9. 168 & 0.634 & 0.010 & W \\
\hline 3737 & 34.671 & 1.160 & 27.801 & & & 0.3 & 0.3 & & & & & $T$ \\
\hline 4135 & 34.685 & & & 0.3 & 0.5 & 0.2 & 0.3 & 0.100 & 0.0130 & 0.032 & 0.010 & 4 \\
\hline 4936 & 34.688 & 1.670 & 27.821 & -0.3 & 0.5 & -0.3 & 0.3 & 0.140 & 0. 841 & 0.030 & 0.009 & 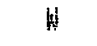 \\
\hline 5335 & 34.679 & 1.460 & 27.787 & 1.1 & 0.2 & 0.9 & 1. 4 & 0.190 & 0.022 & 0.052 & 0.014 & 1 \\
\hline
\end{tabular}

\begin{tabular}{|c|c|}
\hline 0513 & $\mathrm{NC \textrm {i }} / \mathrm{km}^{2} 2$ \\
\hline SRSO FKOH O IO TO $2909 \mathrm{H}=$ & 82.3 aci $/ \mathrm{kn}^{\mathrm{N} 2}$ \\
\hline Fl239 FFoH o to $5706 \mathrm{H}=$ & $3.85 \mathrm{mCi} / \mathrm{kP}^{\mathrm{A}} 2$ \\
\hline 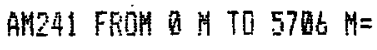 & $1.09-\bar{d} \mathrm{i} / \mathrm{k} \mathrm{m}^{\mathrm{a}} 2$ \\
\hline
\end{tabular}




\begin{tabular}{|c|c|c|c|c|c|c|c|c|c|c|c|}
\hline \multicolumn{2}{|c|}{ CRUISE-STAH } & \multicolumn{2}{|c|}{ POSITION } & \multicolumn{2}{|c|}{ COLLECTION DATE } & \multicolumn{2}{|c|}{ BOTTOH DEPTH } & & & & \\
\hline $6 \%-217$ & $\begin{array}{r}44 \\
17 t\end{array}$ & $\begin{array}{ll}4 & 36.8 \\
4 & 59.2\end{array}$ & $H$ & \multicolumn{2}{|c|}{$10 / 01 / 73$} & \multicolumn{2}{|c|}{5983 月 } & & & & \\
\hline DEFTH & SALIHITY & POT. T & $\begin{array}{l}\text { SIGMAA } \\
\text { THETA }\end{array}$ & \multicolumn{2}{|c|}{ CS137 } & \multicolumn{2}{|c|}{ SR90 } & \multicolumn{2}{|c|}{ PU239 } & A & $\begin{array}{l}\text { LAB } \\
\text { CODE }\end{array}$ \\
\hline \multicolumn{12}{|c|}{ 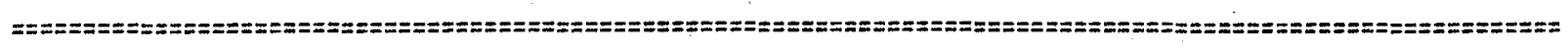 } \\
\hline 5 & 33.940 & 11.100 & 25.273 & 37.5 & 0.3 & 19.8 & 9.2 & & & & $W$ \\
\hline 25 & 33.850 & 10.900 & 25.319 & 37.4 & 0.4 & 21.5 & 0.6 & 0.100 & 0.050 & & H \\
\hline 95 & 33.485 & 5.670 & 26.437 & 33.2 & 0.5 & & & 0.180 & 0.020 & & W \\
\hline 155 & 33.569 & 5.790 & 26.510 & 32.7 & 0.5 & 20.5 & 0.4 & & & & W \\
\hline 236 & 33.904 & 6.850 & 26.721 & 26.7 & 0.5 & & & 0.330 & 0.630 & & H \\
\hline 296 & 33.863 & 5.010 & 26.813 & 24.0 & 0.3 & 16.4 & 0.8 & 0.430 & 0.060 & & in \\
\hline 355 & 33.873 & 4.440 & 26.882 & 17.0 & 0.3 & & & 0.330 & 0.030 & & $H$ \\
\hline 500 & 34.845 & 3.960 & 27.069 & 8.5 & 0.3 & 5.6 & 0.1 & 0.190 & 0.650 & & $H$ \\
\hline 64.5 & 34.182 & 3.790 & 27.293 & 3.7 & 0.3 & & & 0.210 & 0.030 & & 4 \\
\hline 795 & 34.257 & 3.300 & 27.301 & 1.7 & 0.3 & 0.9 & 0.2 & 0.330 & 0.190 & & W \\
\hline 994 & 34.357 & 2.920 & 27.414 & -0.2 & 0.6 & & & 0.270 & 0.070 & & 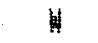 \\
\hline 1142 & 34.414 & 2,640 & 27.484 & 0.2 & 0.3 & -1.2 & 0.4 & & & & $H$ \\
\hline 1499 & 34.599 & 2.220 & 27.595 & -1.8 & 0.3 & 0.0 & 0.6 & & & & $H$ \\
\hline 2085 & 34.585 & 1.740 & 27.692 & -0.4 & 0.3 & -0.7 & 1.0 & & & & $H$ \\
\hline 4142 & 34.667 & 1.120 & 27.800 & 0.2 & 0.2 & & & 0.027 & 0.009 & & $H$ \\
\hline 4935 & 34.685 & 1.100 & 27.816 & 8.4 & 0.5 & & & 0.130 & 0.030 & & $W$ \\
\hline 5538 & 34.653 & 1.100 & 27.791 & -0.1 & 0.2 & 8.5 & 1.2 & 0.091 & 0.018 & & $H$ \\
\hline
\end{tabular}

\begin{tabular}{|c|c|}
\hline 5137 & 65.1 网 $\mathrm{i} / \mathrm{k}^{*} \mathbf{A}^{2}$ \\
\hline 110396 & $46.3 \mathrm{a}$ a $\mathrm{Ci} / \mathrm{k} \cdot \mathrm{AO}^{\mathrm{N}} 2$ \\
\hline 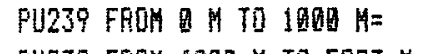 & 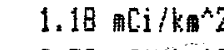 \\
\hline FU239 FROM 4000 M TO 5 & $0.78 \cdot[$ \\
\hline
\end{tabular}




\begin{tabular}{|c|c|c|c|c|c|c|c|c|c|c|c|c|}
\hline \multicolumn{2}{|c|}{ CRUISE-STAI } & \multicolumn{2}{|c|}{ POSITION } & \multicolumn{2}{|c|}{ COLLECTION DATE } & \multicolumn{2}{|c|}{ BOTTOM DEPTH } & & & & & \\
\hline \multirow[t]{2}{*}{$6 x-218$} & \multirow{2}{*}{$\begin{array}{r}50 \\
176\end{array}$} & 26.8 & N & \multirow{2}{*}{\multicolumn{2}{|c|}{$10 / 04 / 73$}} & \multicolumn{2}{|c|}{$7301 \%$} & & & & & \\
\hline & & 36.8 & W & & & & & & & & & \\
\hline \multirow[t]{2}{*}{ DEPTH } & \multirow{2}{*}{ SALIHITY } & \multirow[t]{2}{*}{ POT. T } & \multirow{2}{*}{$\begin{array}{l}\text { SIGMA } \\
\text { THETA }\end{array}$} & \multicolumn{2}{|c|}{ CS137 } & \multicolumn{2}{|c|}{ SR90 } & \multicolumn{2}{|c|}{ PU239 } & \multicolumn{2}{|c|}{ A 241} & LAB \\
\hline & & & & \multicolumn{2}{|c|}{ Gots? } & \multirow{2}{*}{\multicolumn{2}{|c|}{ 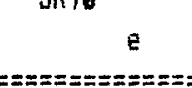 }} & \multirow{2}{*}{\multicolumn{2}{|c|}{$\stackrel{\mathrm{e}}{\mathrm{e}}$}} & \multirow{2}{*}{\multicolumn{2}{|c|}{ 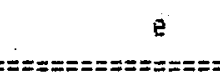 }} & CODE \\
\hline \multicolumn{7}{|c|}{ 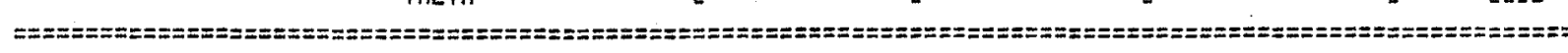 } & & & & & & \\
\hline 5 & 32.669 & 12.060 & 24.815 & 38.7 & 8.4 & 23.3 & 0.8 & 0.040 & 0.010 & 0.014 & 0.014 & $H$ \\
\hline 80 & 33.998 & 3.500 & 27.869 & 34.2 & 0.4 & 18.1 & 0.9 & 0.100 & 0.010 & 0.033 & 0.007 & $H$ \\
\hline 148 & 33.540 & 3.800 & 26.682 & 22.2 & 0.6 & 12.6 & 0.5 & 0.230 & 0.020 & 0.045 & 9.699 & $H$ \\
\hline 195 & 33.717 & 3.580 & 26.845 & 21.6 & 0.6 & 13.1 & 0.2 & 0.250 & 0.020 & 0.059 & 0.912 & $\psi$ \\
\hline 344 & 33.950 & 3.480 & 27.040 & & & 5.8 & 0.3 & 0.250 & 0.030 & 0.079 & 0.011 & $W$ \\
\hline 543 & 34.165 & 3.340 & 27.224 & 4.3 & 0.8 & 3.0 & 0.3 & 0.200 & 0.020 & 0.031 & 0.009 & $H$ \\
\hline 742 & 34.287 & 3.070 & 27.345 & 8.0 & 0.7 & & & 0.198 & 0.618 & & & $\mathrm{~L}$ \\
\hline 992 & 34.381 & & & -0.2 & 0.4 & -0.1 & 0.3 & & & & & $T$ \\
\hline 1290 & 34.467 & 2.340 & 27.551 & 1.3 & 0.6 & & & 0.107 & 0.013 & & & $L$ \\
\hline 1589 & 34.531 & 2.060 & 27.624 & -0.9 & 0.6 & -8.4 & 0.3 & & & & & $T$ \\
\hline 1888 & 34.572 & & & 2.5 & 0.5 & & & 0.058 & 0.009 & & & $L$ \\
\hline 2060 & 34.600 & 1.120 & 27.747 & & & -0.7 & 0.9 & & & & & $\mathrm{I}$ \\
\hline 2195 & 34.596 & 1.798 & & -6.2 & & & & 0.884 & 0.013 & & & L \\
\hline 2593 & 34.637 & 1.470 & 27.753 & 0.5 & 0.6 & 0.5 & 0.4 & & & & & $T$ \\
\hline 2992 & 34.655 & 1.330 & 27.777 & 0.4 & 0.6 & & & 0.055 & 0.009 & & & L \\
\hline 3390 & 34.668 & 1.240 & 27.794 & 0.2 & 0.8 & 1.8 & 0.6 & & & & & $T$ \\
\hline 3799 & 34.675 & 1.160 & 27.904 & -0.2 & & & & 0.060 & 0.009 & & & $L$ \\
\hline 4187 & 34.679 & & & 1.4 & 0.4 & -0.3 & 0.5 & & & & & $T$ \\
\hline 4585 & 34.685 & 1.090 & 27.817 & -0.2 & & & & 0.053 & 0.007 & & & $L$ \\
\hline 4982 & 34.688 & 1. 380 & 27.820 & 1.6 & 0.4 & 1.2 & 0.7 & 0.990 & 0.010 & 0.025 & 0.008 & 1 \\
\hline 5378 & 34.681 & 1.070 & 27.815 & & & & & 0.130 & 0.040 & & & $L$ \\
\hline 6031 & 34,687 & 1.960 & 27.820 & 1.1 & 1.5 & 1.8 & 0.3 & 0.080 & 0.010 & 0.019 & 0.006 & 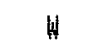 \\
\hline 6433 & 34.685 & & & 1.1 & 1.5 & & & 0.109 & 0.012 & & & $\mathrm{~L}$ \\
\hline 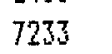 & 34.688 & 1.040 & 27.823 & 0.1 & 0.2 & -9.2 & 0.6 & 0.130 & 0.020 & 0.026 & 0.069 & W \\
\hline
\end{tabular}

CS137 FRIM O IT $1009 \mathrm{H}=$ $5 R 99$ FROH H TO $1000 \mathrm{H}=$ PUISP FROM O $M$ TO $7301 \mathrm{M}=$
$48.5 \mathrm{mCi} / \mathrm{km} 2$

$28.2 \mathrm{mCi} / \mathrm{km} \mathrm{m}^{\wedge}$

3.28 mCi $/ \mathrm{k}^{\circ} \div 2$ 


\begin{tabular}{|c|c|c|c|c|c|}
\hline CFUISE-STA\# & \multicolumn{3}{|c|}{ POSITION } & COLLECTION DATE & BOTTOK DEPTH \\
\hline $5 x-219$ & 5.3 & 6.6 & $N$ & $10 / 08 / 73$ & $3734 \mathrm{H}$ \\
\hline & 177 & 17.5 & H & & \\
\hline
\end{tabular}

\begin{tabular}{|c|c|c|c|c|c|c|c|c|c|c|c|c|}
\hline \multirow[t]{2}{*}{ DEFTH } & \multirow[t]{2}{*}{ SALINITY } & \multirow[t]{2}{*}{ PDT. T } & SIGMA & \multicolumn{2}{|c|}{$\operatorname{cst} 13$} & \multicolumn{2}{|c|}{5890} & \multicolumn{2}{|c|}{ PU239 } & \multicolumn{2}{|c|}{ Al241 } & \multirow{2}{*}{$\begin{array}{l}\text { LAE } \\
\text { CODE }\end{array}$} \\
\hline & & & THETA & & e & & e & & e & & e & \\
\hline \multicolumn{13}{|c|}{ 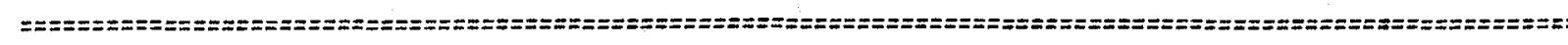 } \\
\hline 70 & 33.070 & 7,300 & 25,990 & 30.3 & 1.2 & 20.5 & 0.9 & & & & & $T$ \\
\hline 70 & 33.340 & 4,300 & 26.476 & 24.0 & D. 5 & 16.1 & 0.2 & 0.110 & 0.020 & 0.028 & 0.099 & 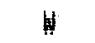 \\
\hline 150 & 33.490 & 3.300 & 26.689 & 23.5 & 0.7 & 16.7 & 0.9 & & & & & $T$ \\
\hline 250 & 33.740 & 3.680 & 26.853 & 20.8 & 1.3 & 11.7 & 0.7 & 0.270 & 0.020 & 0.048 & 1. 3099 & 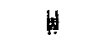 \\
\hline 343 & 37.999 & 3.510 & 27.884 & 11.8 & 1.0 & $b .3$ & 0.8 & & & & & $T$ \\
\hline 494 & 34.882 & 3.360 & 27.156 & 7.1 & 0.7 & 4.8 & 0.2 & 0.200 & 0.020 & 0.039 & 0.699 & H \\
\hline 895 & 34.321 & 2.970 & 27.390 & 0.7 & 0.5 & 1.4 & 0.5 & & & & & $T$ \\
\hline 1244 & $34.42 t$ & 2.480 & 27.507 & -0.2 & 0.3 & B.6 & 0.2 & 0.090 & 0.010 & 0.024 & 0.096 & 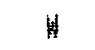 \\
\hline 2739 & 34.641 & 1.440 & 27.759 & 0.8 & 0.7 & -0.2 & 0.5 & & & & & $T$ \\
\hline $323 t$ & 34.658 & 1.360 & 27.778 & -0.8 & 0.7 & 0.0 & 0.2 & 0.090 & 0.810 & 0.969 & 0. 903 & $\omega$ \\
\hline 3702 & 34.662 & 1.290 & 27.796 & -0.2 & 0.4 & 0.2 & 0.6 & & & & & $T$ \\
\hline
\end{tabular}

59137 FROH O 10 T0 3734 H= SF90 FFOM O 10 TO $3734 \mathrm{M}=$

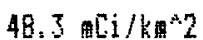

35,3 政 $/ k^{2} 2$ 


\begin{tabular}{|c|c|c|c|c|c|c|c|c|c|c|c|c|}
\hline \multicolumn{2}{|c|}{ CFIISE-STAL } & \multicolumn{2}{|c|}{ P0SITIOH } & \multicolumn{2}{|c|}{ COLLECTIOH DATE } & \multicolumn{2}{|c|}{ BOTTOH DEPTH } & & & & & \\
\hline \multirow[t]{2}{*}{$6 x-222$} & \multirow{2}{*}{$\begin{array}{r}40 \\
160\end{array}$} & 10.0 & $H$ & \multicolumn{2}{|c|}{$10116 / 73$} & \multicolumn{2}{|c|}{$5647 M$} & & & & & \\
\hline & & $0 \quad 30.0$ & $E$ & & & & & & & & & \\
\hline \multirow[t]{2}{*}{ DEFTH } & \multirow[t]{2}{*}{ SALIHITY } & \multirow[t]{2}{*}{ PDT. T } & SIGHA & \multicolumn{2}{|c|}{$[5137$} & \multirow[t]{2}{*}{5690} & \multirow[b]{2}{*}{ e } & \multicolumn{2}{|c|}{ PU239 } & \multicolumn{2}{|c|}{$\operatorname{AM} 241$} & $\angle A E$ \\
\hline & & & THETA & & 2 & & & \multicolumn{2}{|c|}{ g } & \multicolumn{2}{|c|}{ Anst } & COLE \\
\hline \multicolumn{13}{|c|}{ 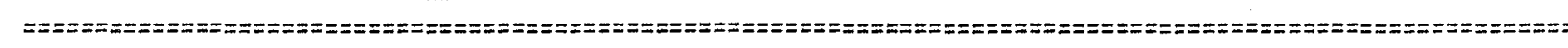 } \\
\hline 10 & 34.130 & 15.700 & 25.176 & 33.3 & 0.4 & 28.4 & 0.7 & 0.092 & 0.014 & & & in \\
\hline 100 & 34.060 & 9.280 & 26.393 & 27.4 & 0.3 & 22.6 & 9.6 & 0.392 & 0.026 & & & H \\
\hline 180 & 33.900 & 6.900 & 26.697 & 28.9 & 0.7 & 22.1 & 0.4 & 0.370 & 0.050 & 0.644 & 0.608 & 嵌 \\
\hline 220 & 33.848 & 6.400 & 36.630 & 27.0 & 0.3 & 18.7 & 0.3 & 0.334 & 0.024 & & & 4 \\
\hline 270 & 33.970 & 5.490 & 26.777 & 24.3 & 0.5 & 17.9 & 0.1 & 0.259 & 0.050 & 0.034 & 0.068 & $H$ \\
\hline 425 & 33.968 & & & 11.6 & 0.2 & 8.6 & 0.7 & 0.252 & 0.821 & & & 4 \\
\hline 540 & 34.129 & & & 6.4 & 0.5 & $4.7^{\circ}$ & 0.5 & 0.240 & 0.830 & 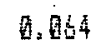 & 0.012 & 4 \\
\hline 295 & 34.213 & 3.540 & 27.246 & 2.3 & 0.6 & 2.0 & 0.2 & & & & & $T$ \\
\hline 845 & 34.294 & 3.180 & 27.341 & 1.7 & 0.3 & 0.6 & 0.1 & 0.170 & 0.620 & 6.69 & 2.069 & 旨 \\
\hline 1050 & 34.390 & 2.790 & 27.452 & 0.1 & 0.5 & -0.2 & 0.4 & & & & & $T$ \\
\hline 1155 & 34.415 & & & 0.2 & 1.0 & 2,1 & 0.5 & 0.110 & 0.620 & 0.055 & ช. & 4 \\
\hline 1810 & 34.509 & 1.940 & 27.657 & 1.1 & 0.5 & -0.1 & 0.4 & & & & & T \\
\hline 1990 & 34.578 & 1.800 & 27.681 & 0.3 & 0.9 & a. 4 & 0.1 & 0.060 & 0. 120 & 0.023 & 9.905 & $H$ \\
\hline 2285 & 34.619 & & & -9.8 & 0.2 & -9.3 & 0.2 & & & & & $T$ \\
\hline 2640 & 34.63 & 1.470 & 27.758 & -9.4 & 0.5 & -8.1 & 0.5 & 0.040 & 0.016 & 0.811 & 9.965 & 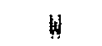 \\
\hline 2815 & 34.652 & & & -0.4 & 1.6 & -0.6 & 0.3 & & & & & $T$ \\
\hline 3965 & 34.642 & & & 0.1 & 0.4 & 0.6 & 0.1 & 0.050 & 0.010 & 0.017 & 0.095 & H \\
\hline 3760 & 34.647 & & & & & 0.5 & 0.3 & & & & & $T$ \\
\hline $47 \pm 9$ & 34.651 & & & -0.4 & 0.3 & 0.3 & 0.2 & 0.080 & 6. 120 & 0.0 .18 & 0.005 & 4 \\
\hline
\end{tabular}

[S13] FFOM O H TO 1000 ShPO FROH of 101090 H= PUZ39 FROM O $\mathrm{H}$ T0 $5647 \mathrm{H}=$

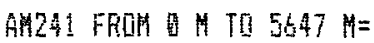

57.6 , ncikgin 2

44.0 mLi $1 / \mathrm{knO}^{\circ} 2$

2.65 G $\mathrm{Ci} / \mathrm{km} 2$

0. $69 \mathrm{mCi} / \mathrm{k}^{\mathrm{2} 2} 2$ 


\begin{tabular}{|c|c|c|c|c|c|c|c|c|c|c|c|c|}
\hline \multicolumn{2}{|c|}{ CRUISE-STAZ } & \multicolumn{2}{|c|}{ P0SITIOH } & \multicolumn{2}{|c|}{ COLLECTION DATE } & \multicolumn{2}{|c|}{ BOTTOM DEPTH } & & & & & \\
\hline GX:-223 & $\begin{array}{r}3 \\
15\end{array}$ & $\begin{array}{ll}84 & 58.4 \\
11 & 50.6\end{array}$ & E & \multicolumn{2}{|c|}{$10 / 20 / 73$} & \multicolumn{2}{|c|}{614011} & & & & & \\
\hline DEPTH & SALINITY & FOT. T & $\begin{array}{l}\text { SIGMA } \\
\text { THETA }\end{array}$ & & {$[5137$} & \multicolumn{2}{|c|}{ SRTQ } & \multicolumn{2}{|c|}{ PU239 } & \multicolumn{2}{|c|}{ All241 } & $\begin{array}{l}\text { LAE } \\
\text { CODE }\end{array}$ \\
\hline \multicolumn{13}{|c|}{ 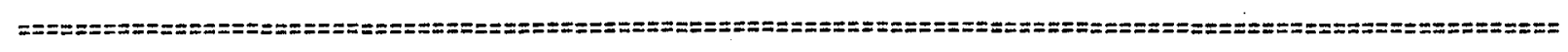 } \\
\hline 10 & & 22.600 & & 41.3 & 1.7 & 29.8 & 1.2 & & & & & T \\
\hline 70 & & 21.200 & & 43.3 & 0.6 & 26.5 & 1.0 & 0.220 & 0.030 & 0.009 & 0.006 & $H$ \\
\hline 125 & 34,790 & 19.000 & 24.944 & 43.3 & 1.8 & 31.0 & 1.3 & & & & & $T$ \\
\hline 200 & 34.770 & 17.400 & 25.275 & $4 \dot{b}, 5$ & 0.5 & 27.2 & 0.2 & 0.320 & 0.030 & 0.072 & 0.015 & $H$ \\
\hline 300 & 34.740 & 16.500 & 25.447 & 48.3 & 1.0 & & & & & & & $T$ \\
\hline 397 & 34.690 & 15.700 & 25.608 & 43.5 & 0.4 & 25.3 & 1.4 & 9.450 & 0.830 & 0.070 & 0.614 & $W$ \\
\hline 547 & 34.424 & 11.900 & 26.199 & 29.7 & 1.0 & & & 0.450 & 0.050 & & & $L$ \\
\hline 697 & 34.223 & 8.110 & 26.688 & 18.5 & 0.3 & 13.5 & 8.4 & 0.410 & 0. 840 & 0.130 & 0.020 & in \\
\hline 897 & 34.076 & 4,350 & 27.053 & 12.9 & 1.0 & & & 0.300 & 0.050 & & & $L$ \\
\hline 1097 & 34.261 & 3.640 & 27.272 & 2.6 & 0.2 & 1.7 & 0.4 & 0.170 & 0.020 & 0. 344 & 0.011 & $W$ \\
\hline 1495 & 34.448 & 2.650 & 27.504 & B. & 0.2 & & & 0.150 & 0.020 & 0.048 & 0.090 & $H$ \\
\hline 2091 & 34.577 & 1.900 & 27.673 & -0.4 & 0.2 & & & 0.071 & 0.010 & 0.022 & 0.906 & 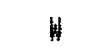 \\
\hline 2685 & $34,63 b$ & 1.550 & 27.745 & 0.3 & 0.2 & 6.7 & & 0.071 & 0.017 & & & $W$ \\
\hline 7205 & 34.658 & 1.340 & 27.778 & 1.8 & 0.2 & 0.1 & 0.2 & 0.065 & 0.010 & 0.021 & $\pi .905$ & 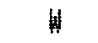 \\
\hline 107 & 34.677 & 1.160 & 27.896 & 0.0 & 0.2 & -0.5 & 0.3 & 0.048 & 0.808 & 0.820 & 0.096 & $W$ \\
\hline 113 & 34.689 & 1.080 & 27.821 & -0.6 & 0.2 & -0.8 & 0.3 & $0.8 B 6$ & 0.015 & 0.050 & 1..699 & $H$ \\
\hline
\end{tabular}

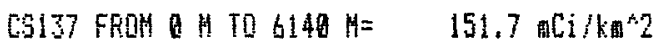

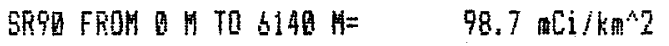

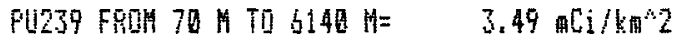

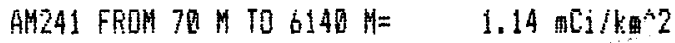




\begin{tabular}{|c|c|c|c|c|c|c|c|c|c|c|c|}
\hline \multicolumn{2}{|c|}{ CEUISE-STA\# } & \multicolumn{2}{|c|}{ PosITION } & \multicolumn{2}{|c|}{ COLLECTION DATE } & \multicolumn{2}{|c|}{ BOTTOM DEPTH } & & & & \\
\hline \multirow[t]{2}{*}{$6 y-224$} & 3 & 1415.6 & N & \multicolumn{2}{|c|}{$16 / 24 / 73$} & \multicolumn{2}{|c|}{$9198 \mathrm{H}$} & \multirow{2}{*}{\multicolumn{2}{|c|}{. }} & & \\
\hline & 14 & 158.8 & $\mathrm{E}$. & & & & & & & & \\
\hline \multirow[t]{2}{*}{ DEPTH } & \multirow[t]{2}{*}{ SALINITY } & \multirow[t]{2}{*}{ POT. T } & SIGMA & \multicolumn{2}{|c|}{65137} & \multicolumn{2}{|c|}{$5 F 90$} & \multicolumn{2}{|c|}{ Fu23: } & A & $\angle A B$ \\
\hline & & & THETA & & e & & e & & $\mathrm{e}$ & e & CONE \\
\hline \multicolumn{12}{|c|}{ 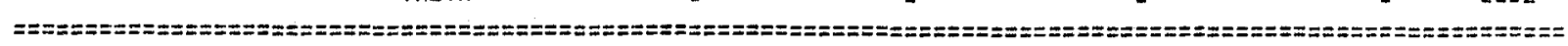 } \\
\hline 20 & 34.500 & 23,890 & 23.349 & 39.5 & 0.3 & 29.5 & 0.5 & 0.120 & 6.020 & & 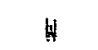 \\
\hline 150 & 34.780 & 18.800 & 24.933 & 41.8 & 0.3 & 27.7 & 1.3 & Q.240 & 0.030 & & W \\
\hline 250 & 34.769 & $17.2 \mathrm{Qg}$ & 25.322 & & & 32.8 & 0.7 & & & & $T$ \\
\hline 400 & 34,692 & 15.730 & 25.602 & 42.9 & 0.3 & 32.7 & 1.3 & 0.770 & 0.030 & & $H$ \\
\hline 500 & 34.539 & 13.560 & 25.957 & 40.0 & 2.0 & 28.9 & 0.9 & & & & $T$ \\
\hline 800 & 34.292 & 5.670 & 26.983 & 9.7 & 0.2 & 7.9 & 0.5 & 0.340 & 0.630 & & 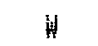 \\
\hline 1345 & 34,408 & 3.970 & 27.441 & 0.7 & 0.2 & 0.5 & 0.2 & & & & H \\
\hline 2243 & 34.691 & 1.760 & 27.703 & 0.3 & 0.2 & -0.4 & 0.4 & 9.190 & 8.003 & & if \\
\hline 3729 & 34.674 & 1.221 & 27.800 & -0.1 & 0.3 & 0.7 & & 0.062 & 0.014 & & 4 \\
\hline 5211 & 34,685 & 1.280 & 27.818 & $A, 8$ & 0.2 & & & 0.850 & 0.010 & & 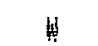 \\
\hline
\end{tabular}

CS137 FROH II TO 250 Th= SR90 FROM I TO $2590 M=$

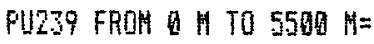

$142.4 \mathrm{ACi} / \mathrm{km} 2$

$105.2 \mathrm{aCi} / \mathrm{k}^{2} 2$

$3.65 \mathrm{mCl} / \mathrm{km}^{\wedge} 2$ 


$\begin{array}{lrcccc}\text { CFUISE-5TA\# } & & \text { FOSITION } & \text { COLLECTIOH DATE } & \text { BOTTON DEPTH } \\ \text { GX-225 } & 32 & 37.0 & \text { N } & 11 / 06 / 73 & 5958 \mathrm{M} \\ & 161 & 55.0 & \mathrm{E} & & \end{array}$

\begin{tabular}{|c|c|c|c|c|c|c|c|c|c|c|c|}
\hline DEFTH & SALINITY & P0T. T & $\begin{array}{l}\text { SIGHA } \\
\text { THETA }\end{array}$ & \multicolumn{2}{|c|}{65137} & \multicolumn{2}{|c|}{$5 R 90$} & \multicolumn{2}{|c|}{ Fu239 } & AM241 & $\begin{array}{l}\text { LAB } \\
\text { CODE }\end{array}$ \\
\hline$=====$ & $:=:= \pm=$ & $=2=8$ & $==x==-=$ & $y=z=z=$ & $y===-$ & $=z= \pm=z$ & $y===2$ & $y=x=x=0$ & $z=z=z=8$ & 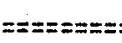 & $== \pm== \pm=$ \\
\hline 10 & 34.560 & 23.580 & 23.484 & 42.0 & 1.3 & 19.9 & 1.0 & 0.055 & 0.010 & & $I$ \\
\hline 50 & 34.590 & 22.100 & 23.904 & & & 31.3 & 1.0 & & & & $T$ \\
\hline 90 & 34.740 & 19.908 & 25.103 & 42.9 & 2.2 & 29.1 & 1.7 & 0.290 & 0.030 & & 2 \\
\hline 130 & 34.720 & 16.990 & 25.353 & & & 30.9 & 1.9 & & & & $\mathrm{~T}$ \\
\hline 180 & 34.760 & 16.380 & 25.487 & 45.5 & 1.9 & 38.9 & 0.6 & 0.275 & 0.011 & & $z$ \\
\hline 250 & 34.680 & 15.690 & 25.631 & & & 31.3 & 1.3 & & & & $T$ \\
\hline 350 & 34.560 & 13.800 & 25.914 & 43.1 & 0.9 & 38.3 & 1.6 & 0.475 & 0.029 & & $?$ \\
\hline 399 & 34.490 & 13.100 & 26.014 & & & 27.5 & 1.4 & & & & $T$ \\
\hline 599 & $34: 114$ & 8.170 & 26.593 & 27.5 & 0.9 & 18.8 & 1.2 & 1. 424 & 0.025 & & 2 \\
\hline 895 & 34.138 & 4.240 & 27.114 & & & 3.2 & 0.8 & & & & $\mathrm{~T}$ \\
\hline 1191 & 34.354 & 3.170 & 27.390 & 0.2 & 0.5 & 1.2 & 0.5 & 0.160 & 0.927 & & $I$ \\
\hline 1489 & 34,471 & 2,490 & 27.543 & & & 0.1 & 0.5 & & & & ! \\
\hline 1999 & 34.586 & 1.840 & 27.685 & -8.2 & & & & 9.991 & 0.604 & & L \\
\hline 2487 & 34.631 & 1.540 & 27.743 & & & 0.2 & 1.4 & & & & $T$ \\
\hline 2692 & 34.649 & 1.440 & 27.764 & -10.2 & & & & 9.102 & 0.030 & & $L$ \\
\hline 3187 & 34.66 & 1.290 & 27.788 & & & -0.2 & 0.3 & & & & $T$ \\
\hline 3684 & 34.673 & 1.190 & 27.801 & & & -8.6 & 0.2 & 0.073 & 0.809 & & I \\
\hline 4180 & 34.682 & 1.130 & 27.812 & & & 0.1 & 0.2 & & & & $T$ \\
\hline 4679 & 34.687 & 1.986 & 27.819 & -7.2 & & -0.1 & 0.2 & 0.100 & 0.012 & & 2 \\
\hline 5177 & 34.681 & 1.030 & 27.817 & & & 1.7 & 0.4 & & & & $T$ \\
\hline 508 & 34.689 & 0.991 & 27.827 & 0.2 & 0.6 & 1.4 & 0.5 & 9.150 & 0.020 & & 7 \\
\hline
\end{tabular}

CS137 FROA D H TO 159A H= 5F90 FROM O I1 10 15R日 $\mathrm{H}=$ Fli239 FROA 9 M T0 5958 H=
$145.8 \mathrm{mCj} / \mathrm{km} 2$

$92.5 \mathrm{mCj} / \mathrm{kn}^{\circ} \mathrm{z}$

$4.04 \mathrm{mCj} / \mathrm{k}$. 2 


\begin{tabular}{|c|c|c|c|c|c|c|c|c|c|c|c|}
\hline \multicolumn{2}{|c|}{ CRUISE-STA\# } & \multicolumn{2}{|c|}{ POSITION } & \multicolumn{2}{|c|}{ COLLECTION DATE } & \multicolumn{2}{|c|}{ BOTTOM DEPTH } & & & & \\
\hline $5 \times-226$ & 3 & 34.0 & $H$ & \multicolumn{2}{|c|}{$11 / 09 / 73$} & \multicolumn{2}{|c|}{5603} & & & & \\
\hline \multirow[t]{2}{*}{ DEPTH } & \multirow[t]{2}{*}{ SALIHITY } & \multirow[t]{2}{*}{ POT. T } & SIGMA & \multicolumn{2}{|c|}{$\operatorname{cs137}$} & \multicolumn{2}{|c|}{ SR9Q } & \multicolumn{2}{|c|}{ PL1239 } & $A M 241$ & $\angle A B$ \\
\hline & & & THETA & & e & & e & & e & e & CODE \\
\hline \multicolumn{12}{|c|}{ 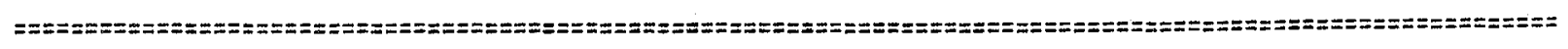 } \\
\hline 19 & 35.930 & 24.700 & 23.475 & 44.5 & 0.4 & 27.8 & 0.3 & 0.140 & 0.010 & & $H$ \\
\hline 40 & 35.030 & 24.700 & 23.482 & 46.4 & 1.0 & 32.5 & 0.7 & & & & $T$ \\
\hline 90 & 34.850 & 23.700 & 23.647 & 50.5 & 0.1 & 26.6 & 0.3 & 0.110 & 0.010 & & H. \\
\hline 150 & 34.730 & 16.280 & 25.529 & 48.6 & 1.0 & 36.4 & 1.9 & & & & $T$ \\
\hline 250 & 34.580 & 14.490 & 25. 9.96 & 43.6 & 0.4 & 29.1 & 0.3 & 0.430 & 0.030 & & $H$ \\
\hline 350 & 34.430 & 13.200 & 25.950 & 35.3 & 2.8 & 30.1 & 1.2 & 1.530 & 0.048 & & 1 \\
\hline 452 & 34.292 & 10.450 & 26.360 & 35.0 & 0.3 & 18.0 & 0.6 & 0.510 & 0.048 & & 其 \\
\hline 533 & 34.147 & 8.560 & 26.560 & 27.7 & 1.4 & 20.4 & 0.4 & 0.480 & 0.030 & & $k$ \\
\hline 345 & 34.642 & 6.410 & 26.784 & 17.2 & 0.3 & 8.9 & 0.5 & 0.480 & 0.030 & & in \\
\hline 984 & 34.211 & 3.900 & 27.206 & 0.6 & 0.4 & 0.3 & 0.6 & & & & $T$ \\
\hline 1192 & 34.403 & 3.050 & 27.439 & 0.1 & 0.2 & -0.2 & 0.3 & 0.170 & 0.020 & & $H$ \\
\hline 1489 & 34.500 & 3.380 & 27.487 & 0.6 & 0.4 & 0.1 & 0.4 & & & & $T$ \\
\hline 2499 & 34.642 & 1.460 & 27.758 & & & 0.1 & 0.3 & & & & $T$ \\
\hline 3495 & 34.673 & 1.200 & 27.800 & -8.2 & 0.3 & & & 0.031 & 0.015 & & W \\
\hline 4499 & 34.697 & 1.060 & 27.821 & -1.1 & 0.5 & -8.1 & 0.5 & & & & $T$ \\
\hline 5434 & 34.688 & 0.970 & 27.827 & -0.5 & 0.4 & 0.0 & 0.3 & & & & $T$ \\
\hline
\end{tabular}

[S137 FFDH O 14 To $5603 \mathrm{M}=$

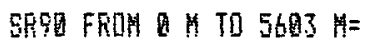

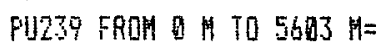

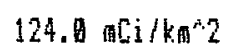

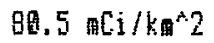

$3.27 \mathrm{Ci} / \mathrm{km} 2$ 


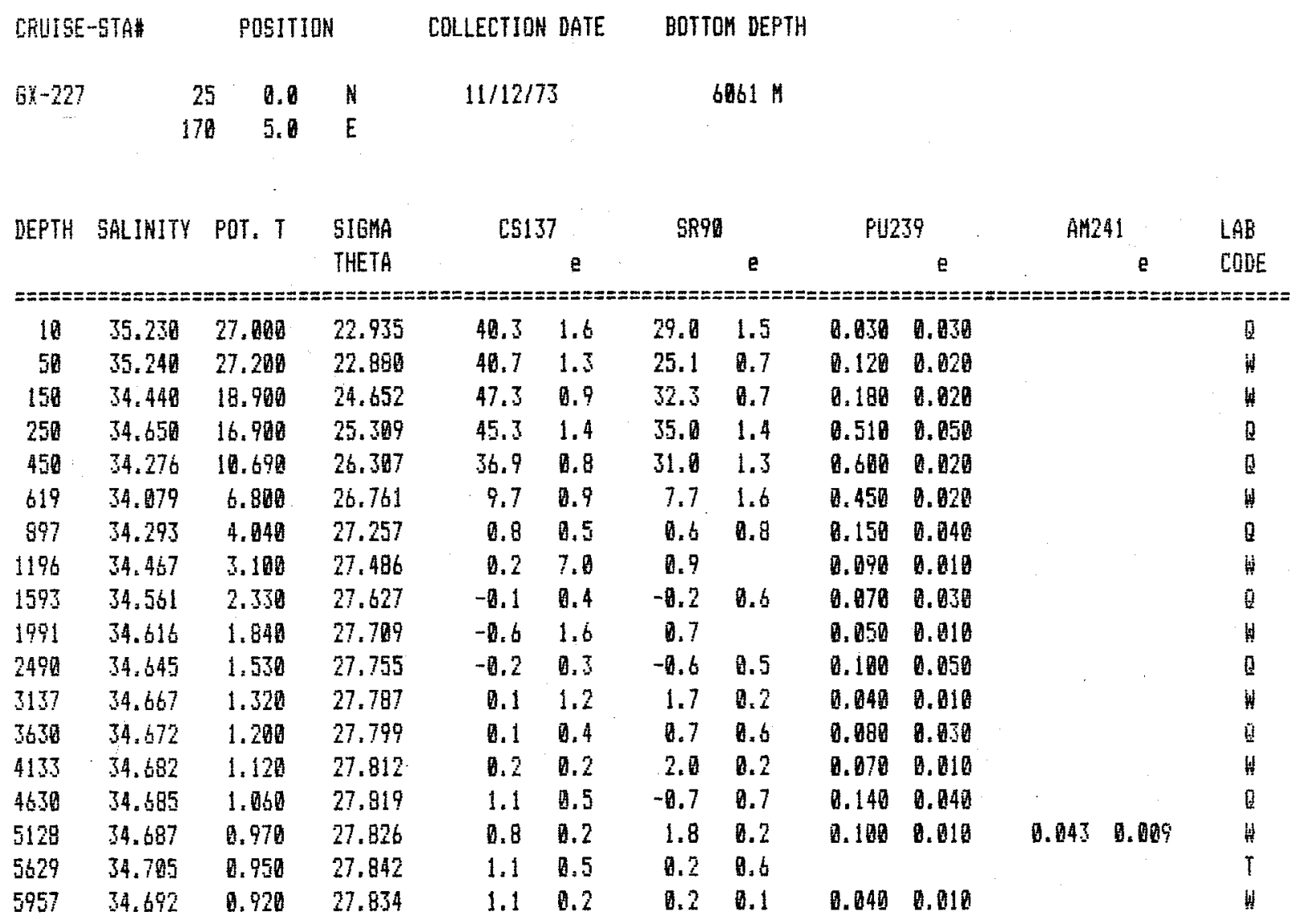

OS137 FFOM I $M$ TO $1509 \mathrm{M}=$ SR90 FROM O $\mathrm{H}$ TO $1500 \mathrm{H}=$

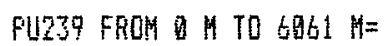

112. $\mathrm{mCl} / \mathrm{km}^{2} 2$

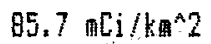

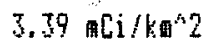




\begin{tabular}{|c|c|c|c|c|c|c|c|c|c|c|c|}
\hline \multicolumn{2}{|c|}{ CFUISE-STAH } & \multicolumn{2}{|c|}{ Position } & \multirow{2}{*}{\multicolumn{2}{|c|}{$\begin{array}{l}\text { COLLECTIOH DATE } \\
11 / 18 / 73\end{array}$}} & \multirow{2}{*}{\multicolumn{2}{|c|}{$\begin{array}{c}\text { BOTTOM DEPTH } \\
5729 \mathrm{M}\end{array}$}} & & & & \\
\hline $6 x-229$ & 17 & $\begin{array}{ll}12 & 53.0 \\
73 & 28.0\end{array}$ & $\begin{array}{l}N \\
E\end{array}$ & & & & & & & & \\
\hline DEPTH & SALIHITY & POT. T & $\begin{array}{l}\text { SIGMA } \\
\text { THETA }\end{array}$ & & 55137 & \multicolumn{2}{|c|}{ SR90 } & \multicolumn{2}{|c|}{ PU239 } & $\begin{array}{r}\mathrm{A} M 241 \\
\mathrm{e}\end{array}$ & $\begin{array}{l}\text { LAE } \\
\text { CODE }\end{array}$ \\
\hline$:====$ & 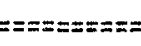 & $=z=x=z=z=$ & $y=z=z===$ & =:=:=:=:=3 & $:=====$ & & $:==$ & $y=====$ & $==z= \pm=$ & $y=z==:==$ & $== \pm==3$ \\
\hline 10 & 34.440 & 27.990 & 22.052 & 27.9 & 1.2 & & & 0.049 & 8.807 & & 1 \\
\hline 50 & 34.580 & 27.500 & 22.161 & & & 19.6 & 0.8 & & & & $T$ \\
\hline 70 & 34.621 & 27.870 & 22.199 & 31.7 & 1.4 & & & 0.029 & 0.089 & & $L$ \\
\hline 172 & 34.949 & 20.160 & 24.696 & 64.2 & 1.3 & 41.2 & 1.7 & & & & $T$ \\
\hline 249 & 35.905 & 13.650 & 26.299 & 53.3 & 1.1 & & & 0.293 & 0.023 & & $L$ \\
\hline 345 & 34.313 & 9.596 & 26.524 & & & 9.0 & 0.5 & & & & $T$ \\
\hline 444 & 34,348 & 7.310 & 26.903 & 19.4 & 0.6 & & & 0.331 & 0.023 & & $L$ \\
\hline 693 & 34.490 & 5.480 & 27.253 & -1.0 & 0.7 & 1.5 & $B .4$ & & & & $T$ \\
\hline 993 & 34.546 & 4.160 & 27.446 & -9.2 & & & & 0.296 & 0.023 & & $L$ \\
\hline 1291 & 34.579 & 3.230 & 27.563 & & & 0.5 & 0.6 & & & & $T$ \\
\hline 1590 & 34.602 & 2.590 & 27.638 & -9.2 & & & & 0.159 & 0.013 & & $\mathrm{~L}$ \\
\hline 1888 & 34.626 & 2.150 & 27.693 & & & 0.1 & 0.2 & & & & $T$ \\
\hline 2286 & 34.645 & 1.800 & 27.736 & -0.2 & & & & 0.093 & 0.013 & & $L$ \\
\hline 2684 & 34.662 & 1.530 & 27.769 & & & 0.2 & 0.7 & & & & $T$ \\
\hline 3184 & 34.674 & 1.340 & 27.792 & -10.2 & & & & 0.053 & 0.010 & & $L$ \\
\hline 3684 & 34.682 & 1.170 & 27.809 & -1.6 & 0.8 & 0.3 & 0.2 & & & & $T$ \\
\hline 4196 & 34.691 & 1.060 & 27.824 & 2.0 & 0.9 & & & 0.029 & 0.006 & & $L$ \\
\hline 4686 & 34.697 & 0.940 & 27.836 & & & -8.3 & 0.3 & & & & $T$ \\
\hline 5189 & 34.792 & 0.890 & 27.843 & -0.2 & & & & B. 044 & 0.011 & & $L$ \\
\hline 5689 & 34.696 & 0.850 & 27.841 & & & 0.8 & 0.3 & & & & $T$ \\
\hline
\end{tabular}

C\$177 FROM O M TO $1990 \mathrm{M}=$ SRPO FFOA OH TO $1000 \mathrm{M}=$ PU239 FROH H TO $5729 \mathrm{H}=$
$94.5 \times \mathrm{Cj} / \mathrm{km} \div 2$

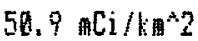

2.95 - 


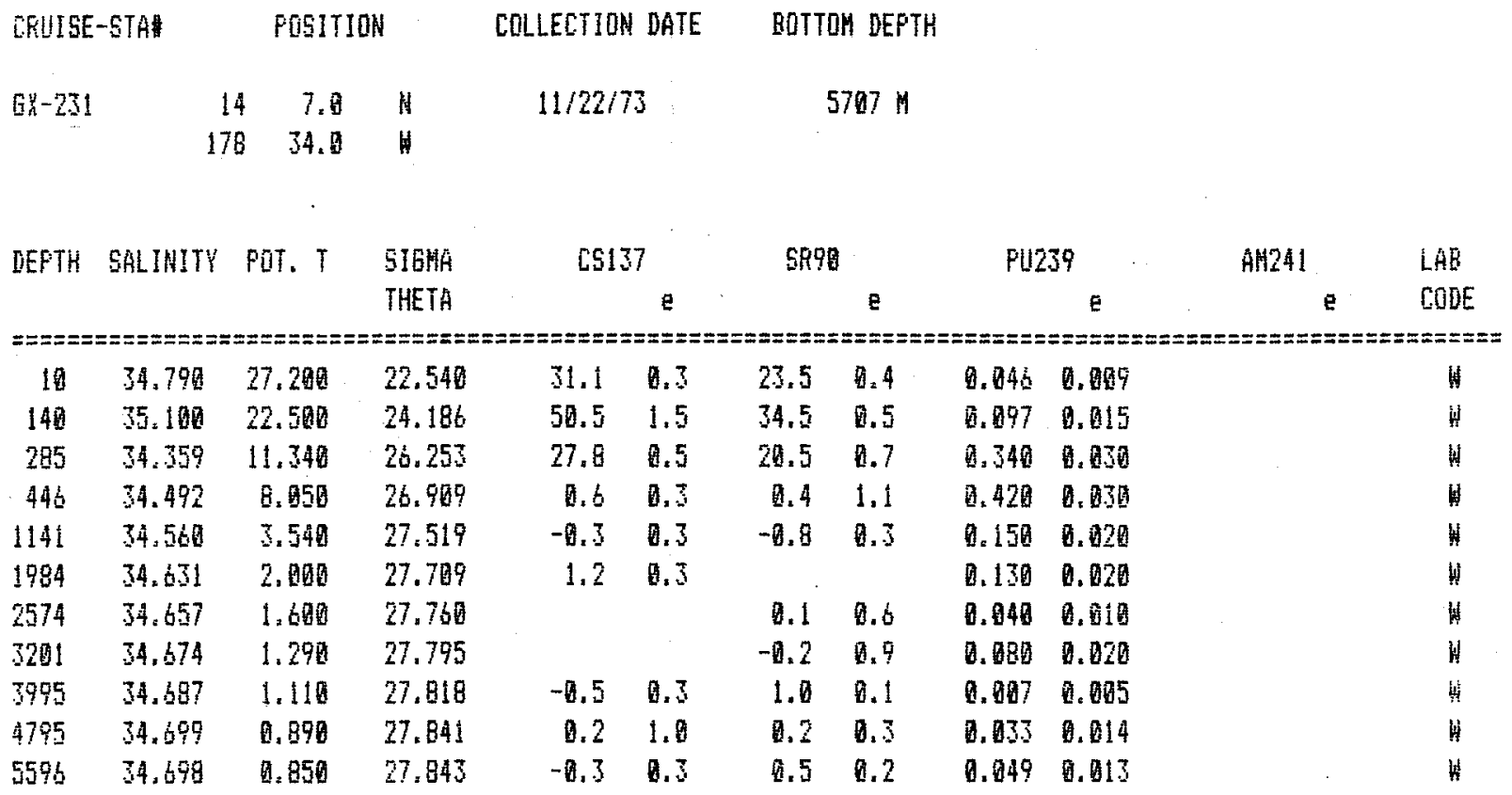

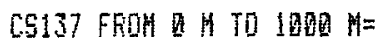

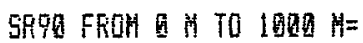

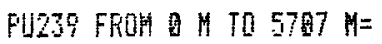

$62.1 \propto\left[\mathrm{i} / \mathrm{km}^{* 2}\right.$

$44.2 \mathrm{mCi} / \mathrm{km}^{\mathrm{N} 2}$

$2.68 \mathrm{mCj} / \mathrm{kn}^{\wedge} 2$ 


\begin{tabular}{|c|c|c|c|c|c|}
\hline CFUISE-STA\# & \multicolumn{3}{|c|}{ P0SITIOH } & COLLECTION DATE & BOTTOH DEFTH \\
\hline \multirow[t]{2}{*}{$6 \mathrm{H}-235$} & 16 & 45.4 & $N$ & $12 / 46 / 73$ & 5564 \\
\hline & 151 & 23.7 & 4 & & \\
\hline
\end{tabular}

\begin{tabular}{|c|c|c|c|c|c|c|c|c|c|c|c|}
\hline DEPTH & SALINITY & POT. $\mathrm{Y}$ & SIGMA & $\mathrm{CSI}$ & & SF9 & & Fu? & & $A M 241$ & $\angle A B$ \\
\hline & & & THETA & & e & & e & & e & e & CODE \\
\hline$=====$ & 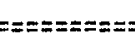 & $==ニ=ニ=ニ=$ & $== \pm==ニ+=$ & $z== \pm=$ & $E===$ & $z=z==0$ & $z=== \pm$ & $z==z==z$ & $== \pm== \pm=$ & $==ニ=\pi=z=$ & $z=z=z=z$ \\
\hline 10 & 34,310 & 25,890 & 22.610 & 32.9 & 1.0 & & & 0.060 & 0.920 & & $L$ \\
\hline 49 & 34.398 & 25.780 & 22.799 & 33.5 & 1.4 & 24.7 & 1.0 & & & & $T$ \\
\hline 70 & 34.610 & 25.990 & 22.897 & 35.5 & 1.0 & & & 0.075 & 0.015 & & $\mathrm{~L}$ \\
\hline 130 & 34.906 & 21.200 & 24.397 & & & 37.1 & $1 . \overline{7}$ & & & & T! \\
\hline 275 & 34.213 & 10.629 & 26.270 & 31.9 & 1.0 & & & 0.420 & 9.621 & & $L$ \\
\hline 314 & 34.217 & 9.540 & 26.454 & & & 12.0 & 0.7 & & & & $T$ \\
\hline 354 & 34.188 & 9.230 & 25.643 & 5.6 & 1.0 & & & 0.730 & 0. 640 & & 1 \\
\hline 495 & 34.399 & 6.840 & 27.000 & & & 1.0 & 0.5 & & & & $T$ \\
\hline 592 & 34.445 & 6.190 & 27.136 & & & & & 0.469 & 0.060 & & $L$ \\
\hline 496 & 34,464 & 5.810 & 27.192 & -0.1 & 0.3 & 1.6 & 0.4 & & & & $T$ \\
\hline 1090 & 34,534 & 4.040 & 27.448 & & & & & 0.110 & 0.039 & & $\hat{L}$ \\
\hline 1496 & 34.589 & 2.890 & 27.610 & & & 0.8 & 0.2 & & & & $\mathrm{i}$ \\
\hline 1894 & 34,623 & 2.990 & 27.696 & & & & & 0.030 & 0.020 & & $L$ \\
\hline 2993 & 34,641 & 1.730 & 27.737 & & & 0.2 & 0.3 & & & & ז \\
\hline 3165 & 34.672 & 1.330 & 27.791 & & & -1.2 & 0.2 & & & & $T$ \\
\hline 3505 & 34.679 & 1.220 & 27.804 & & & & & 0.020 & 0.020 & & $L$ \\
\hline 3905 & 34.682 & 1.140 & 27.811 & 0.6 & 0.6 & 0.3 & 0.4 & & & & $T$ \\
\hline 4797 & 34.692 & 1.800 & 27.828 & & & 0.3 & 0.7 & & & & $T$ \\
\hline 5108 & 34.696 & 0.960 & 27.834 & & & & & 0.013 & 0.016 & & $L$ \\
\hline 5510 & 34.691 & 0.920 & 27.833 & & & 0.8 & 面. 3 & & & & $T$ \\
\hline
\end{tabular}

CS137 FFon 11 TO 1600 H= SR9O FFOA O $\mathrm{H}$ TO $1000 \mathrm{H}=$

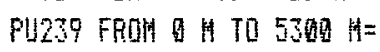

$52.7 \mathrm{mCl} / \mathrm{km} 2$ $46.0 \mathrm{mi} / \mathrm{km}^{*} 2$

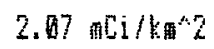




\begin{tabular}{|c|c|c|c|c|c|c|c|c|c|c|c|}
\hline \multicolumn{2}{|c|}{ CRUISE-STA\# } & \multicolumn{2}{|c|}{ POSITION } & \multicolumn{2}{|c|}{ COLLECTION DATE } & \multicolumn{2}{|c|}{ BOTTOH DEPTH } & & & & \\
\hline $6 x-23$ & 17 & $\begin{array}{rr}5 & 53.0 \\
2 & 0.9\end{array}$ & $H$ & $12 / 12 /$ & & & $868 \mathrm{H}$ & & & & \\
\hline DEPTH & SALIHITY & Por. T & $\begin{array}{l}\text { SIGMA } \\
\text { THETA }\end{array}$ & $\operatorname{csi}$ & & SR9R & e & $\mathrm{Pu} 2$ & & AH241 & LAE \\
\hline$==$ & $:==z==z=$ & $z===x+$ & $== \pm==-3$ & $z==z==$ & $== \pm==8$ & $=z== \pm=$ & $==== \pm$ & $:== \pm= \pm==$ & $:=x==x$ & $=x=x=x$ & $x====$ \\
\hline 10 & 35.930 & 25.890 & 23.151 & 24.5 & 0.5 & 22.2 & 1.6 & 0.958 & 0.020 & & Q \\
\hline 50 & 35.030 & 25.500 & 23.247 & 21.7 & 0.4 & 16.1 & 0.4 & 0.041 & 0.011 & & $H$ \\
\hline 89 & 35.158 & 25.410 & 23.367 & 22.3 & 1.4 & 17.4 & 1.9 & 9.840 & 0.930 & & $\theta$ \\
\hline 125 & 35.330 & 25.470 & 23,478 & 21.0 & 0.7 & 14,3 & 0.4 & 0.052 & 0.014 & & $H$ \\
\hline 295 & 34.936 & 19.840 & 24.696 & 35.3 & 1.8 & 18.8 & 1.0 & 0.990 & 0.030 & & 8 \\
\hline 293 & 34.649 & 10.420 & 26.644 & 4.6 & 0.3 & 2.8 & 0.5 & 0.172 & 0.905 & & H \\
\hline 440 & 34.635 & 8.640 & 26.931 & 0.2 & $B .6$ & 0.7 & 0.8 & 0.289 & 0.070 & & 0 \\
\hline 637 & 34.571 & 7.879 & 27.112 & -0.9 & 0.3 & & & 0.223 & 0.030 & & W \\
\hline 865 & 34.545 & 5.230 & 27.326 & & & & & 0.248 & 0.846 & & L \\
\hline 1044 & 34.560 & 4.280 & 27.445 & -0.3 & 0.2 & 0.8 & & 0.106 & 0.015 & & W \\
\hline 1693 & 34.616 & 2.590 & 27.650 & -0.3 & 0.2 & 1.9 & & 0.039 & 6.097 & & 4 \\
\hline 2491 & 34.658 & 1.690 & 27.754 & 0.4 & 0.3 & 1.1 & & 0.033 & 0.999 & & 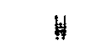 \\
\hline 3290 & 34.676 & 1.310 & 27.795 & -8.6 & 0.2 & 0.7 & & 0.945 & 6.011 & & $W$ \\
\hline 3690 & 34.683 & 1.190 & 27.809 & & & 0.3 & 0.3 & 0.334 & 0.013 & & $\psi$ \\
\hline 4140 & 34.692 & 1.000 & 27.828 & & & 9.7 & 0.5 & 0.041 & 0.812 & & 4 \\
\hline 5041 & 34.697 & 0.850 & 27.841 & & & & & 0.117 & 0.024 & & $H$ \\
\hline
\end{tabular}

CS137 FFIM O TO $750 \mathrm{M=}$ SF9 FFOM DI TO $750 \mathrm{H}=$

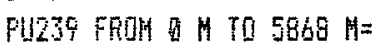

32,4 的i $/ \mathrm{km}^{\wedge} 2$

$22.3 \mathrm{mC \textrm {j }} / \mathrm{km}^{\mathrm{A}} \mathrm{2}$

$2.24 \mathrm{mCi} / \mathrm{km}^{\wedge} \mathrm{2}$ 


\begin{tabular}{|c|c|c|c|c|c|c|c|c|c|c|c|}
\hline CFUISE & -STA & POSIT & & COLLECT10 & DATE & BOTा & DEPTH & & & & \\
\hline $6 x-241$ & & 33,6 & 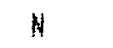 & $12 / 17 /$ & & & 727 1 & & & & \\
\hline & 17 & 0.2 & $E$ & & & & & & & & \\
\hline DEFIH & SALIHITY & POT. T & S16MA & 651 & & Sp? & & Fil2 & & $4 n_{4}$ & 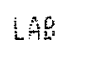 \\
\hline & & & THETA & & e & & e & & e & e & MDE \\
\hline$== \pm==$ & $z==:=z=z=z$ & $==z==z=z$ & $z=z== \pm=z=$ & $===\pi=z==z$ & $=z= \pm=z$ & $z=z== \pm$ & $===z=$ & $z== \pm=z=$ & $===z=z=$ & 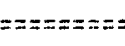 & $z==z=-x$ \\
\hline 5 & 35.097 & 27.170 & 22.774 & 26.5 & 1.1 & 20.0 & 1.3 & 0.100 & 3.050 & & 9 \\
\hline 50 & 35.114 & 27.160 & 22.794 & & & 17.3 & 1.1 & & & & $T$ \\
\hline 150 & 35.230 & 26.310 & 23.147 & 24.8 & 9.5 & $17 . \overline{8}$ & 1.1 & 9.850 & 9.930 & & $\tilde{y}$ \\
\hline 230 & 34.558 & 14.040 & 25,872 & 29.5 & $0 . \overline{9}$ & 22.5 & 0.5 & & & & 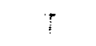 \\
\hline 295 & 34.346 & 9.560 & 25.796 & 1.4 & 0.4 & 1.6 & 0.5 & 0.260 & 0,040 & & 9 \\
\hline 549 & 34.583 & 7,310 & 27.898 & 0.5 & 0.4 & 0.5 & $0 . \bar{E}$ & 0.210 & 0.950 & & 3 \\
\hline 674 & 34.560 & 6.410 & 27.192 & & & 1,1 & 9.7 & & & & $i$ \\
\hline 5670 & 34.649 & 0.810 & 27.846 & -0.2 & 0.5 & & & 0.850 & $A .020$ & & $\vdots$ \\
\hline
\end{tabular}

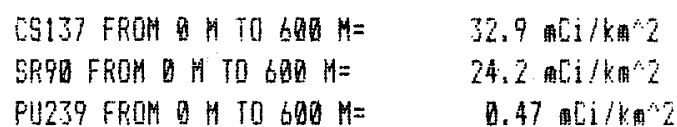

\begin{tabular}{|c|c|c|c|c|c|}
\hline ERUISE-STA\# & \multicolumn{3}{|c|}{ POSITIOH } & COLLECTION DATE & BOTTOH DEFTH \\
\hline $5 x-24 b$ & $\theta$ & 0.9 & 5 & $12 / 21 / 73$ & 5422 \\
\hline & 178 & 59.0 & $E$ & & \\
\hline
\end{tabular}

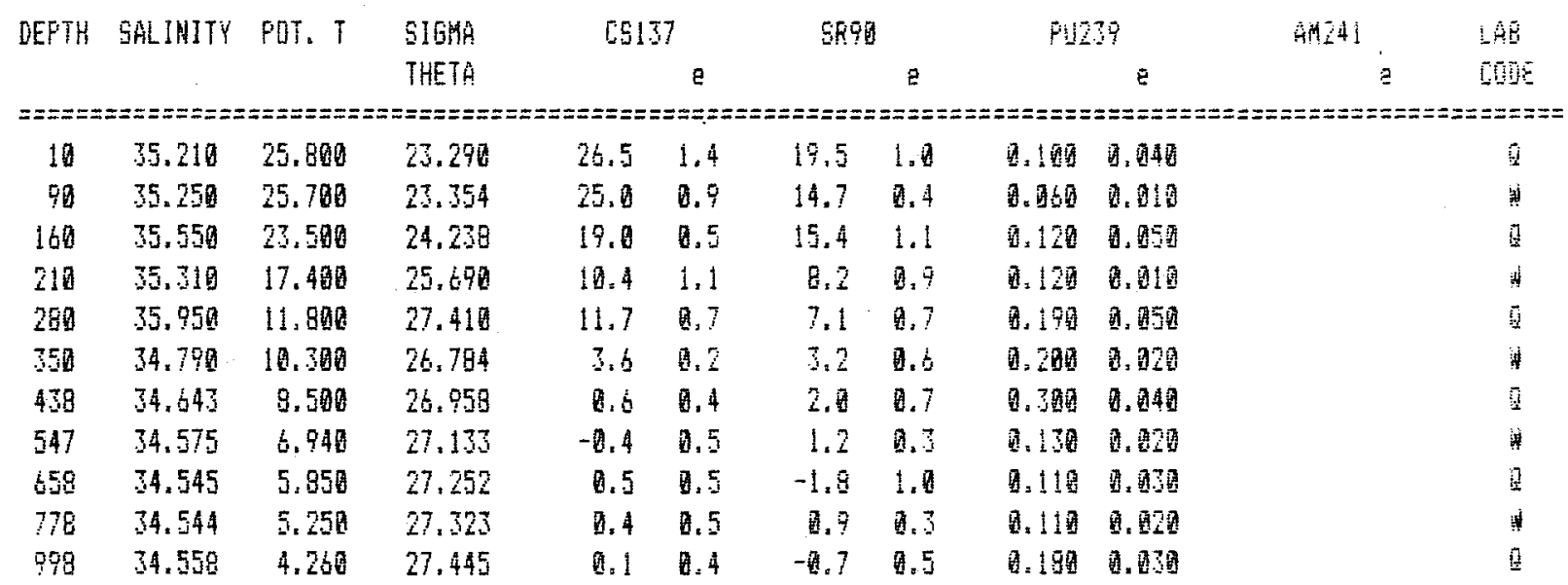

05137 FHUH O H TO 500 H

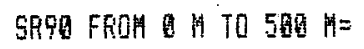

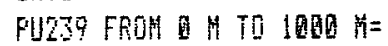

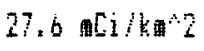

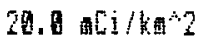

0.67 a $1 \mathrm{~kg}$ 


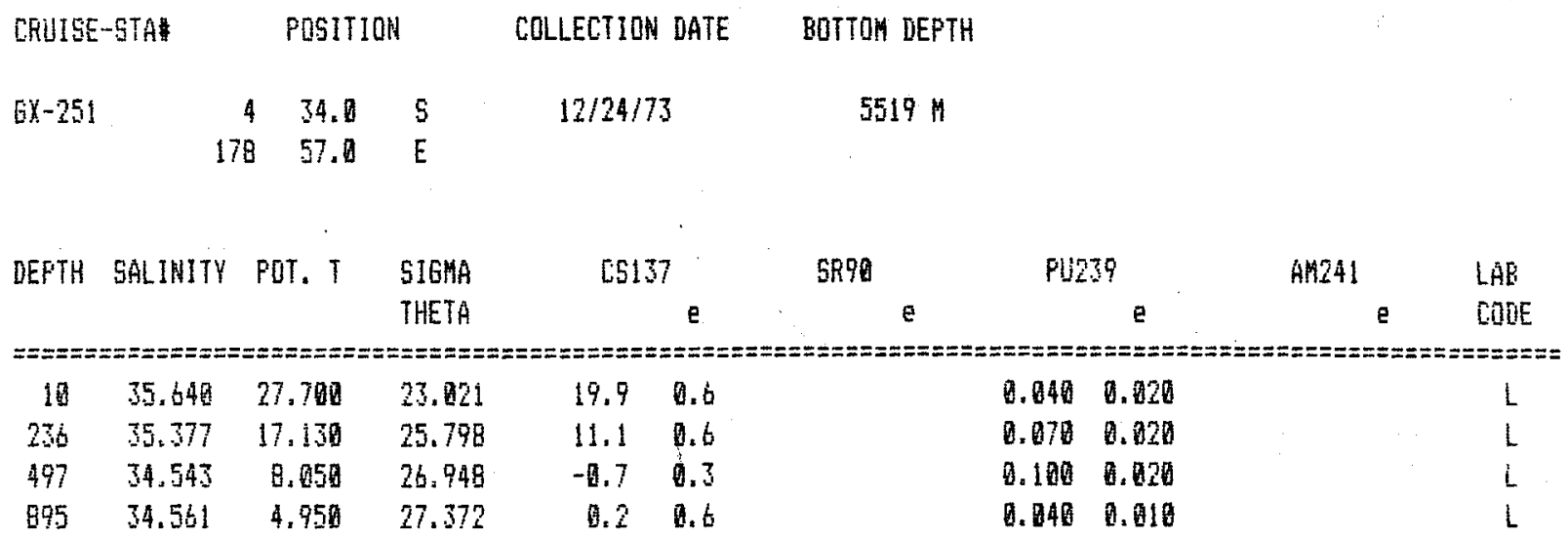

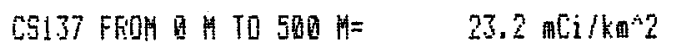

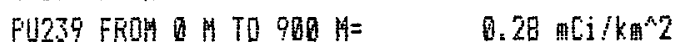

CRUISE-STA\# POSITION GOLLCTIOH DATE BOTTOM DEPTH

$\begin{array}{rrrrrr}6 y-263 & 16 & 41.9 & 5 & 01 / 10 / 74 & 571614 \\ & 167 & 3.7 & \text { H } & \end{array}$

\begin{tabular}{|c|c|c|c|c|c|c|c|c|c|c|c|}
\hline DEPTH & SALIHITY & PDT. T & $\begin{array}{l}\text { SIGHA } \\
\text { THETA }\end{array}$ & \multicolumn{2}{|c|}{$\cos 137$} & \multicolumn{2}{|c|}{$5 R 90$} & \multicolumn{2}{|c|}{ PU2 39} & AM241 & $\begin{array}{l}\angle A E \\
\text { CODE }\end{array}$ \\
\hline \multicolumn{12}{|c|}{ 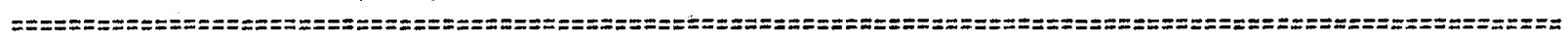 } \\
\hline 40 & 34.679 & 28.480 & 22.071 & 21.6 & 0.4 & 14.0 & 0.6 & 0.040 & 0.810 & & W \\
\hline 150 & 35.810 & 23.180 & 24.550 & 17.4 & 0.3 & 7.1 & 0.2 & 0.050 & 0.010 & & 4 \\
\hline 348 & 35,142 & 15.490 & 26.003 & 15.1 & 0.6 & 6. & 1.2 & 0.120 & 0.020 & & 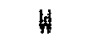 \\
\hline 590 & 34.373 & 5.400 & $27.64 t$ & -6.6 & 6.3 & 0.7 & & 9.930 & 0.010 & & H \\
\hline 3666 & 34.699 & 1.280 & 27.812 & 0.3 & 9.4 & 2.1 & & 0.020 & Q.01日 & & $W$ \\
\hline 4465 & 34.709 & 0.990 & 27.855 & -0.6 & 0.2 & -9.5 & 0.6 & 0.018 & & & ty \\
\hline 5676 & 34.7199 & 0.640 & 27.965 & 0.3 & Q.6 & 0.7 & & 0.018 & & & W \\
\hline
\end{tabular}

C5137 FROA O $M$ T0 6 BO $H=$ SFPQ FROM 11 TO $609 \mathrm{H}=$ PL239 FBOA M TO $5716 \mathrm{~N}=$

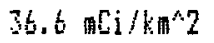

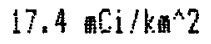

0.65 畞 $/ \mathrm{k}^{*} 2$ 


\begin{tabular}{|c|c|c|c|c|c|c|c|c|c|c|c|}
\hline \multicolumn{2}{|c|}{ CRUISE-STAZ } & \multicolumn{2}{|c|}{ POSITION } & \multicolumn{2}{|c|}{ COLLECTION DATE } & \multicolumn{2}{|c|}{ BOTTOH DEPTH } & & & & \\
\hline $6 x-326$ & $\begin{array}{r}1 \\
12\end{array}$ & $\begin{array}{rr}4 & 3.3 \\
6 & 15.7\end{array}$ & 5 & \multicolumn{2}{|c|}{$85 / 20 / 74$} & \multicolumn{2}{|c|}{$3923 \mathrm{H}$} & & & & \\
\hline \multirow[t]{2}{*}{ DEPTH } & \multirow[t]{2}{*}{ SALINITY } & \multirow[t]{2}{*}{ POT. T } & S16KA & \multicolumn{2}{|c|}{ C5137 } & \multicolumn{2}{|c|}{ SR9O } & \multicolumn{2}{|c|}{ PU1239 } & AM241 & LAE \\
\hline & & & THETA & & a & & e & & e & e & CDOE \\
\hline \multicolumn{12}{|c|}{ 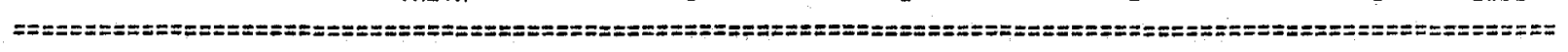 } \\
\hline 10 & 35.620 & 21.490 & 24.677 & & & 16.0 & 1.4 & & & & $T$ \\
\hline 110 & 36.430 & 24.200 & 24.696 & 20.5 & 1.1 & 12.0 & 0.7 & & & & $T$ \\
\hline 197 & 35.797 & 20.410 & 25.281 & 19.4 & 0.6 & & & 0.320 & 8. 040 & & 0 \\
\hline 599 & 34.520 & 6.260 & 27.180 & 0.3 & B.5 & & & 9.070 & 0.030 & & $\underline{0}$ \\
\hline 799 & 34.508 & 5.810 & 27.323 & 0.0 & 0.4 & -0.5 & 0.5 & & & & $\mathrm{~T}$ \\
\hline 1047 & 34.531 & 4.800 & 27.450 & 1.3 & 0.4 & 0.2 & 0.8 & 0.084 & 6.004 & & 0 \\
\hline 1295 & 34.567 & 3.210 & 27.555 & 0.1 & 0.4 & -1.0 & 1.0 & & & & $T$ \\
\hline 1546 & 34.595 & 2.698 & 27.625 & -0.4 & 0.4 & -1.4 & 0,8 & 9.900 & 9.020 & & a \\
\hline
\end{tabular}

\begin{tabular}{|c|c|c|c|c|c|c|c|c|c|c|c|}
\hline \multicolumn{2}{|c|}{ CEUISE-STAZ } & \multicolumn{2}{|c|}{ PDSITION } & \multicolumn{2}{|c|}{ COLLECTION DATE } & \multicolumn{2}{|c|}{ BOTTOM DEPTH } & & & & \\
\hline $6 x^{2}-328$ & 1 & $\begin{array}{ll}9 & 16.9 \\
35\end{array}$ & 5 & \multicolumn{2}{|c|}{$05 / 21 / 74$} & \multicolumn{2}{|c|}{4989} & & & & \\
\hline DEFTH & SALIHITY & POT. T & $\begin{array}{l}\text { SIGMA } \\
\text { THETA }\end{array}$ & \multicolumn{2}{|c|}{ c\$137 } & SR90 & 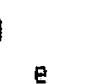 & \multicolumn{2}{|c|}{ PU239 } & AM241 & $\begin{array}{l}\text { LAE } \\
\text { CODE }\end{array}$ \\
\hline \multicolumn{12}{|c|}{ 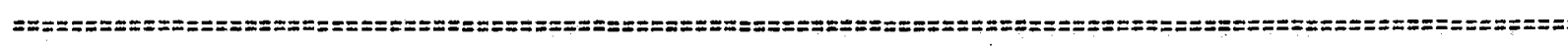 } \\
\hline 1930 & 34.626 & 2.330 & 27.679 & -0.4 & 0.4 & 0.1 & 0.5 & & & & $T$ \\
\hline 2028 & 34.642 & 2.040 & 27.714 & 0.1 & 0.4 & 0.0 & 0.7 & 0.080 & 0.048 & & 0 \\
\hline 2277 & 34.660 & 1.810 & 27.747 & -0.5 & 0.4 & 0.4 & 0.7 & & & & $T$ \\
\hline 2527 & 34.670 & 1,660 & 27.766 & B. 9 & 0.5 & 1.9 & 1.8 & 0.000 & 0.020 & & 0 \\
\hline 2776 & 34,675 & 1.590 & 27.775 & 0.1 & 0.7 & -0.6 & 0.4 & & & & $T$ \\
\hline 3826 & 34.680 & 1.470 & 27.787 & & & & & 0.040 & 0.020 & & $L$ \\
\hline 3276 & $34: 694$ & 1.350 & 27.799 & 0.4 & 0.4 & 0.7 & 0.9 & & & & $T$ \\
\hline 3527 & 34.689 & 1.240 & 27.818 & & & & & 0.020 & 0.018 & & $L$ \\
\hline 3829 & 34.690 & 1.190 & 27.815 & 0.3 & 0.4 & -0.5 & 0.8 & & & & $T$ \\
\hline
\end{tabular}




\begin{tabular}{|c|c|c|c|c|c|c|c|c|c|c|c|}
\hline \multicolumn{2}{|c|}{ CFUISE-GTA\# } & \multicolumn{2}{|c|}{ Position } & \multicolumn{2}{|c|}{ COLLECTIOH DATE } & \multicolumn{2}{|c|}{ BOTTOM DEPTH } & & & & \\
\hline \multirow[t]{2}{*}{$6 x-331$} & \multirow{2}{*}{$\begin{array}{r}4 \\
125\end{array}$} & 36.6 & 5 & \multicolumn{2}{|c|}{$05 / 24 / 74$} & \multirow{2}{*}{\multicolumn{2}{|c|}{$4490 \mathrm{M}$}} & & & & \\
\hline & & 8.5 & 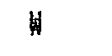 & & & & & & & & \\
\hline \multirow[t]{2}{*}{ DEPTH } & \multirow[t]{2}{*}{ SALINITY } & \multirow[t]{2}{*}{ FOT. T } & \multirow{2}{*}{$\begin{array}{l}\text { SIGMA } \\
\text { THETA }\end{array}$} & \multicolumn{2}{|c|}{$[9137$} & \multicolumn{2}{|c|}{$\sin 9$} & \multicolumn{2}{|c|}{ PU239 } & A 1241 & LAE \\
\hline & & & & & \multirow{2}{*}{ e } & & \multirow{2}{*}{$\begin{array}{l}\mathrm{e} \\
== \pm= \pm=\end{array}$} & & $\mathrm{e}$ & $\mathrm{e}$ & CODE \\
\hline \multicolumn{10}{|c|}{ 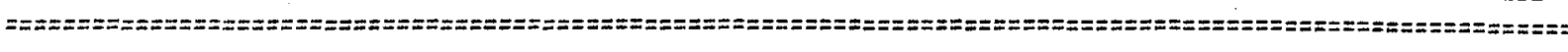 } & & \\
\hline 10 & 35.140 & 26.390 & 23. 192 & 16.5 & 0.9 & 11.2 & 0.6 & & & & $T$ \\
\hline$B 0$ & 35.450 & 26.280 & 23,351 & 14.6 & 0.8 & & & 0.070 & 0.030 & & 9 \\
\hline 90 & 35.330 & 22.100 & 24.467 & 14.9 & 0.8 & 8.8 & 0.6 & & & & $\mathrm{~T}$ \\
\hline 100 & 35.200 & 18.200 & 25.404 & 11.8 & 0.6 & & & 0.020 & 0.020 & & 0 \\
\hline 240 & 34.880 & 11.900 & 26.559 & 1.1 & 0.5 & 0.3 & 0.7 & & & & T⿱ \\
\hline 302 & 34.816 & 10.980 & 26.675 & 1.0 & 0.4 & 0.1 & 1.0 & 0.130 & 0.030 & & 0 \\
\hline 382 & 34.729 & 9.58 . & 26.852 & 0.1 & 0.5 & 0.3 & 0.6 & & & & $T$ \\
\hline 593 & 34.624 & 7.970 & 27.024 & 9.0 & 0.5 & -0.2 & 1.3 & 0.080 & 0.830 & & Q \\
\hline 632 & 34.570 & b.489 & 27.190 & & & -0.6 & 0.5 & & & & $T$ \\
\hline 798 & 34.542 & 5.260 & 27.321 & -0.2 & 0,4 & 1.1 & 0.7 & 0.020 & 0.020 & & $\theta$ \\
\hline 1999 & 34.564 & 4.990 & 27.467 & & & -8.3 & 0.4 & & & & $T$ \\
\hline 1400 & 34.591 & 3.200 & $27.57 \mathrm{t}$ & -6.7 & 0.4 & 0.0 & 0.5 & & & & $T$ \\
\hline 1701 & 34.616 & 2.570 & 27.651 & 1.0 & 0.5 & 1.5 & 0.8 & 0.900 & 0.020 & & 0 \\
\hline 2002 & 34.640 & 2.145 & 27.705 & 0.1 & 0.4 & 1.1 & 2.1 & & & & $T$ \\
\hline 3265 & 34.686 & 1.270 & 27 . & 7. & 0.4 & 0.4 & 0.9 & 9.030 & 0.030 & & 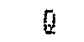 \\
\hline 3647 & 34.692 & 1.120 & 27.820 & & & 0.2 & 0.6 & & & & $\mathrm{~T}$ \\
\hline 4ast & 34.694 & 1.100 & 27.824 & 0.1 & 0.4 & 9.0 & 0.7 & & & & $T$ \\
\hline 4467 & 34.692 & 1.100 & 27.822 & -0.2 & 0.4 & -0.3 & 0.4 & & & & $T$ \\
\hline
\end{tabular}

CS137 FROM O TO $590 \mathrm{~K}=$ SR9O FFOM O $M$ TO 5 SO $M=$ PU239 FFOY O TO $4490 \mathrm{H}=$
$11.5 \mathrm{mLi} / \mathrm{km} 2$

$7.4 \mathrm{aCi} / \mathrm{km} 2$

0.57 a 


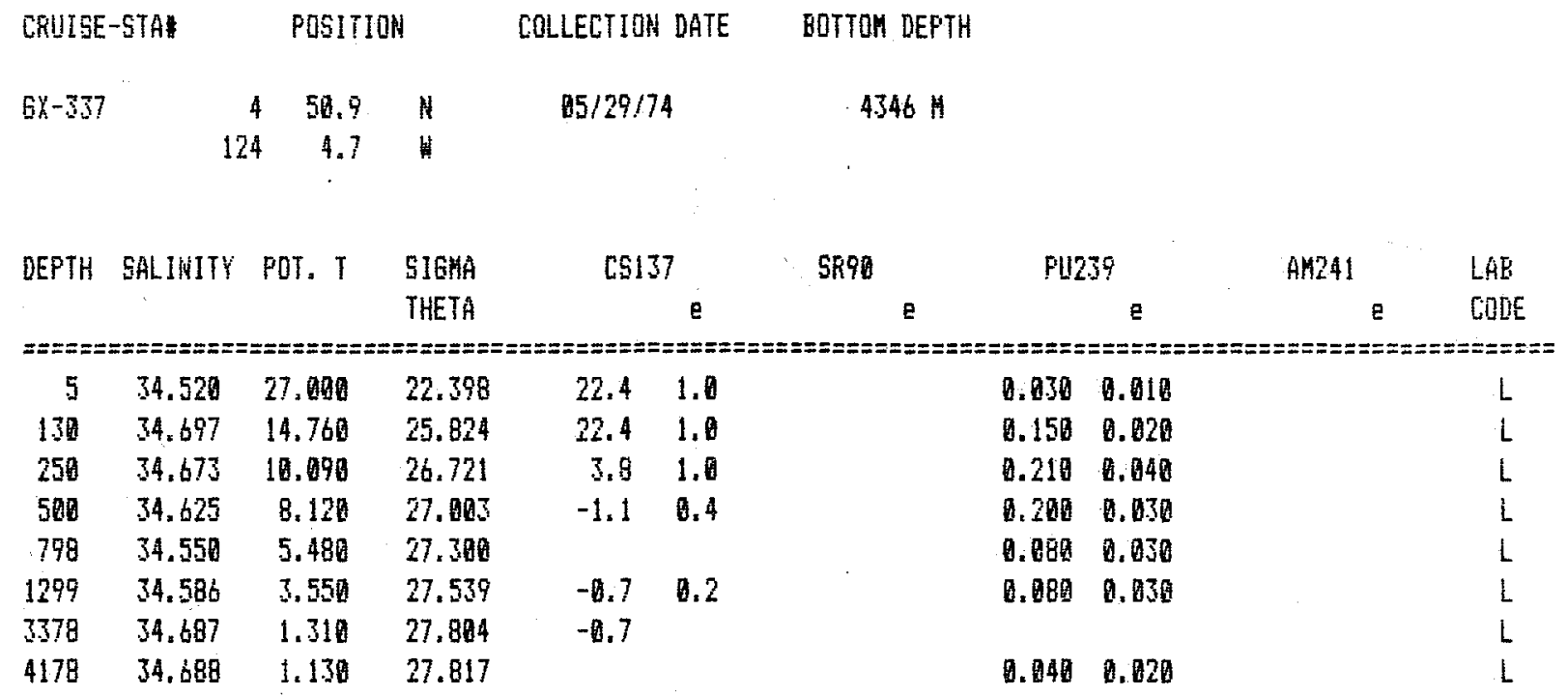

CS137 FROA O H TO 500 $M=$ FU23: FROM O 1 TO $4346 \mathrm{~K}=$
$22.3 \mathrm{mCi} / \mathrm{kg}^{\wedge} 2$

$1.56 \curvearrowleft \mathrm{mi} / \mathrm{kg}^{\mathrm{2}} 2$ 


\begin{tabular}{|c|c|c|c|c|c|c|c|c|c|c|c|}
\hline \multicolumn{2}{|c|}{ CRUISE-STAH } & \multicolumn{2}{|c|}{ PUSITIOH } & \multicolumn{2}{|c|}{ CQLLECTION DATE } & \multicolumn{2}{|c|}{ BOTTOM DEPTH } & & & & \\
\hline \multirow[t]{2}{*}{$6 x-343$} & \multirow[b]{2}{*}{12} & $6 \quad 31.3$ & $H$ & \multirow{2}{*}{\multicolumn{2}{|c|}{$06 / 03 / 74$}} & \multirow{2}{*}{\multicolumn{2}{|c|}{42624}} & & & & \\
\hline & & 259.5 & W & & & & & & & & \\
\hline \multirow[t]{2}{*}{ DEFTH } & \multirow[t]{2}{*}{ 5ALIMITY } & \multirow[t]{2}{*}{ FOT. i } & \multirow{2}{*}{$\begin{array}{l}\text { SIGMA } \\
\text { THETA }\end{array}$} & \multicolumn{2}{|c|}{$\operatorname{css} 137$} & \multicolumn{2}{|c|}{5990} & \multicolumn{2}{|c|}{ PU239 } & AM241 & LAB \\
\hline & & & & & \multirow{2}{*}{$\mathrm{e}$} & & \multirow{2}{*}{$\stackrel{\mathrm{e}}{==z=z=}$} & \multicolumn{2}{|l|}{ 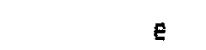 } & e & CUDE \\
\hline \multicolumn{10}{|c|}{ 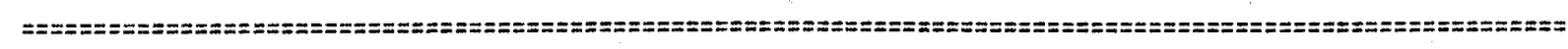 } & & \\
\hline 10 & 34.619 & 22.500 & 23.805 & 41.5 & 0.7 & 27.2 & 0.9 & 0.020 & 0.010 & & W \\
\hline 120 & 34.670 & 18.890 & 24.392 & 56.5 & 0.4 & 31.9 & 1.7 & 0.110 & 0.010 & & $y$ \\
\hline 200 & 34.700 & 11.800 & 26.437 & 5.2 & 0.3 & 2.7 & & 0.120 & 0. 010 & & 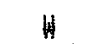 \\
\hline 300 & 34.660 & 10.300 & 26.691 & 1.9 & 0.2 & 9.7 & & 8.110 & 0.010 & & $H$ \\
\hline 450 & 34.522 & 7.790 & 26.971 & -0.3 & 0.4 & 0.7 & & 0.150 & 0.810 & & $H$ \\
\hline 797 & 34.518 & 5.917 & 27.331 & 0.6 & 0.5 & 1.5 & & 0.070 & 0.010 & & 4 \\
\hline 995 & 34.539 & 4.240 & 27.432 & 0.2 & 0.2 & 1.3 & 0.3 & 8.070 & 0.010 & & $H$ \\
\hline 1251 & 34.557 & 3.480 & 27.530 & & & 0.5 & 0.2 & 0.030 & 0.010 & & $H$ \\
\hline 1499 & 34.595 & 2.830 & 27.612 & 0.5 & 0.2 & 1.7 & 0.5 & 0.050 & 0.010 & & $H$ \\
\hline 1746 & 34.620 & 2.370 & 27.671 & 0.4 & 0.5 & 0.4 & 0.3 & Q.860 & 0.010 & & $H$ \\
\hline 2240 & 34.652 & $1.75 a$ & 27.745 & 2.4 & 0.5 & 2,1 & & 0.030 & 0.010 & & H \\
\hline 3403 & 34.678 & 1.280 & 27.799 & 1.3 & 0.9 & 1.8 & & 0.060 & 0.010 & & $W$ \\
\hline 3653 & 34.681 & 1.240 & 27.804 & 0.6 & 0.4 & 1.5 & & 0.020 & 0.610 & & 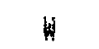 \\
\hline 4153 & 34.684 & 1.170 & 27.811 & 1.1 & 0.2 & 1.5 & & 0.950 & 0.010 & & $H$ \\
\hline
\end{tabular}

[S137 FROM O T TO $450 \mathrm{H}=39.5 \mathrm{ACi} / \mathrm{km}^{\circ} 2$

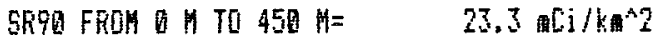

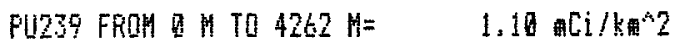

CFUISE-STA\# PDSITIOH COLLECTION DATE BOTTOM DEPTH

G*-347 $\quad 28 \quad 39.9 \quad \mathrm{~N} \quad 06 / 88 / 74 . \quad 4293 \mathrm{M}$

12129.2 W

\begin{tabular}{|c|c|c|c|c|c|c|c|c|c|c|}
\hline \multirow[t]{2}{*}{$T H$} & \multirow[t]{2}{*}{ SALINITY } & \multirow[t]{2}{*}{ POT. T } & \multirow{2}{*}{ 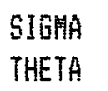 } & \multicolumn{2}{|c|}{ [S137 } & \multirow[t]{2}{*}{$5 R 90$} & \multicolumn{2}{|c|}{ FU239 } & \multirow{2}{*}{$\mathrm{AML}_{41}$} & \multirow{2}{*}{$\begin{array}{l}\angle A B \\
\text { CODE }\end{array}$} \\
\hline & & & & & E & & & $\mathrm{E}$ & & \\
\hline \multicolumn{11}{|c|}{ 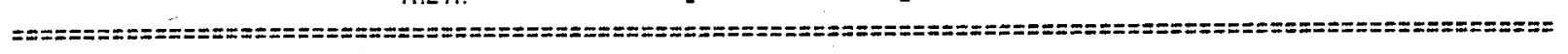 } \\
\hline 5 & 33.650 & 17.100 & 24.484 & 57.3 & 1.0 & & 0.030 & 0.010 & & $L$ \\
\hline 599 & 34.407 & 5.980 & 27.127 & -0.4 & 0.3 & & 0.060 & 0.020 & & $L$ \\
\hline 01 & 34.581 & 2.820 & 27.602 & & & & 0.040 & 0.020 & & L \\
\hline 93 & 34.633 & 1.998 & 27.712 & 0. 2 & 0.7 & & 0.020 & 0.020 & & 1 \\
\hline
\end{tabular}




\section{DOCUMENT LIBRARY}

April 9, 1985

DISTRIBUTION LIST FOR TECHNICAL REPORT EXCHANGE

Institute of Marine Sciences Library

University of Alaska

O'Neill Building

905 Koyukuk Ave., North

Fairbanks. AK

Attn: Stella Sanchez-Wade

Documents Section

Scripps Institution of Oceanography

Library, Mail Code C-075C

La Jolla, CA 92093

Hancock Library of Biology \& Oceanography

Alan Hancock Laboratory

University of Southern California

University Park

Los Angeles, CA 90089-0371

Gifts \& Exchanges

Library

Bedford Institute of Oceanography

P.O. Box 1006

Dartmouth, NS, B2Y 4A2, CANADA

Office of the International

Ice Patrol

c/ o Coast Guard R \& D Center

Avery Point

Groton, CT 06340

Library

Physical Oceanographic Laboratory

Nova University

8000 N. Ocean Drive

Dania, FL 33304

NOAA EDIS Miami Library Center 4301 Rickenbacker Causeway

Miami, FL 33149

\section{Library}

Skidaway Institute of Oceanography

P.O. Box 13687

Savannah, GA 31416

Institute of Geophysics

University of Hawaii

Library Room 252

2525 Correa Road

Honolulu, HI 96822

\section{Library}

Chesapeake Bay Institute

4800 Atwell Road

Shady Side, MD 20876
MIT Libraries

Serial Journal Room 14E-210

Cambridge, MA 02139

Director, Ralph M. Parsons Laboratory

Room 48-311

MIT

Cambridge, MA 02139

Marine Resources Information Center

Bldg. E38-320

MIT

Cambridge, MA 02139

Library

Lamont-Doherty Geological Observatory

Colombia University

Palisades, NY 10964

Library

Serials Department

Oregon State University

Corvallis, OR 97331

Pell Marine Science Library

University of Rhode Island

Narragansett Bay Campus

Narragansett, RI 02882

Working Collection

Texas A\&M University

Dept. of Oceanography

College Station, TX 77843

Library

Virginia Institute of Marine Science

Gloucester Point, VA 23062

Fisheries-Oceanography Library

151 Oceanography Teaching Bldg.

University of Washington

Seattle, WA 98195

Library

R.S.M.A.S.

University of Miami

4600 Rickenbacker Causeway

Miami, FL 33149 
4. Tikle and Subtitle WHOH $-85-19$

Fallout Nuclides in Atlantic and Pacific Water Columns: GEOSECS Data

7. Author(s) H.D. Livingston ${ }^{1}$, V.T. Bowen ${ }^{1}$, S.A. Casso ${ }^{1}$, H.L. Volchok ${ }^{2}$, V.E. Noshkin ${ }^{3}$, K.M. Wong ${ }^{3}$, and T.M. Beasley 4

9. Performipue Organization Name and Address

1. Woods Hole Oceanographic Institution, Woods Hole, MA

2. Environmental Measurements Laboratory, U.S. Dept. of Energy, New York, NY

3. Lawrence Livermore Lab., U. of California, Livermore, CA 4. School of Oceanography, Oregon State U. Newport. OR 97365 12. Sponsoring Organization Arams and Address

United States Department of Energy

Technical

14.

15. Supplementary Notes

This report should be cited as: Woods Hole Oceanog. Inst. Tech. Rept., WHOI-85-19.

16. Abstruct (Limit: 200 words)

This report contains results of measurements of the fallout radionuclides ${ }^{90} \mathrm{Sr},{ }^{137} \mathrm{Cs}, 239,240 \mathrm{Pu}$, and $241 \mathrm{Am}$ in large volume seawater samples collected between 1972 and 1974 in the Atlantic and Pacific as part of Geochemical Ocean Sections (GEOSECS) program. The stations for which data are reported include both the North and South Atlantic oceans and latitudes north of $20^{\circ} \mathrm{S}$ in the Pacific Ocean. The ${ }^{90} \mathrm{Sr}$ and $137 \mathrm{Cs}$ data set has been corrected by a procedure which estimates independently the analytical blank for the laboratory which made the analysis. When the data quality and spacing permit, water column inventory estimates were made for each nuclide over depth intervals appropriate to the nuclide's distribution.

17. Document Analysis a. Descriptors
1. GEOSECS
5. Am-241
2. $\mathrm{CS}-137$
6. Atlantic Ocean
3. $\mathrm{Sr}-90$
7. Pacific Ocean

4. $\mathrm{Pu}-239,240$

b. Identifiers/Open-Ended Terms

c. COSATI Field/Group

12. Availability Statemen:

Approved for publication; distribution unlimited.

\begin{tabular}{|l|l|}
\hline $\begin{array}{l}\text { 19. Secartey Class (This Report) } \\
\text { UNCLASSIFIED }\end{array}$ & $\begin{array}{c}\text { 21. No. of Pages } \\
73\end{array}$ \\
\hline 20. Socurity Class (This Page) & 22. Pric \\
\hline
\end{tabular}

